\title{
POLARIZATION IN PROTON-PROTON SCATTERING
}

\author{
AT $5.15,7.0$ AND $12.33 \mathrm{GeV} / \mathrm{c}^{*+}$
}

Technical Report

G. W. Abshire, C. M. Ankenbrandt ${ }^{\ddagger}$, R. R. Crittenden, D. B. Curtis, R. M. Heinz, K. Hinotani ${ }^{\star \star}$, H. A. Neal ${ }^{\dagger \dagger}$, H. R. Petri, and D. R. Rust

\author{
Physics Department, Indiana University \\ Bloomington, Indiana 47401
}

January 1974

This report was prepared as an account of work sponsored by the United States Government. Neither the United States nor the United States Atomic Energy Commission, nor any of their employees, nor any of their contractors, subcontractors, or their employees, makes any warranty, express or implied, or assumes any legal liability or responsibility for the accuracy com pleteness or usefulness of any information, app, product or process disclosed, or represents that its use would not infringe privately owned rights.

Work supported by the United States Atomic Energy Commission under Contract No. AT (11-1)-2009, Task A, and by the Alfred P. Sloan Foundation.

Portions of this report have been submitted as a thesis to the faculty of the graduate school of Indiana University by Gerald W. Abshire in partial fulfillment of the requirements for the Ph. D. degree.

$\neq$

Present address: National Accelerator Laboratory, Batavia, Illinois.

$\star \star$

On leave from Tenri University, Tenri, Nari, Japan during the 1972-73 academic year.

$+t$ Alfred P. Sloan Foundation Fellow

\section{MASTER}




\section{DISCLAIMER}

This report was prepared as an account of work sponsored by an agency of the United States Government. Neither the United States Government nor any agency Thereof, nor any of their employees, makes any warranty, express or implied, or assumes any legal liability or responsibility for the accuracy, completeness, or usefulness of any information, apparatus, product, or process disclosed, or represents that its use would not infringe privately owned rights. Reference herein to any specific commercial product, process, or service by trade name, trademark, manufacturer, or otherwise does not necessarily constitute or imply its endorsement, recommendation, or favoring by the United States Government or any agency thereof. The views and opinions of authors expressed herein do not necessarily state or reflect those of the United States Government or any agency thereof. 


\section{DISCLAIMER}

Portions of this document may be illegible in electronic image products. Images are produced from the best available original document. 


\section{ABSTRACT}

We present the results of an experiment to measure the polarization in large angle proton-proton elastic scattering. This experiment, performed at Argonne National Laboratory, represents the first high statistics protonproton polarization measurements for $|\mathrm{t}|$ values greater than $2.5(\mathrm{GeV} / \mathrm{c})^{2}$. Using the arguments of Wolfenstein, we show that the proton-proton system is completely specified by five complex amplitudes. We represent the polarization as the product of the helicity amplitudes of Goldberger. Since the polarization is the product of amplitudes, it is very sensitive to the structure of the amplitudes. Thus, these measurements provide meaningful constraints on the amplitudes over a large range of $t$.

A polarized target consisting of a liquid $\mathrm{He}^{4}$ cooled ethylene glycol target in a $25 \mathrm{kG}$ magnetic field produced the polarized protons. The two final state protons were detected in a two arm multiwire proportional chamber spectrometer. Each arm determined the momentum of one of the protons with a resolution of $\pm 5 \%$ and the time-of-flight within . $7 \mathrm{~ns}$. The momentum, time-of-flight, and scattering angle of each proton was used to determine which events were elastic.

The $5.15 \mathrm{GeV} / \mathrm{c}$ polarization measurements covered from $t=-.54$

$(\mathrm{GeV} / \mathrm{c})^{2}$ to $t=4.03(\mathrm{GeV} / \mathrm{c})^{2}$. There is a minimum at $t=-.7(\mathrm{GeV} / \mathrm{c})^{2}$ 
followed by a maximum of .22 at $t=-1.8(\mathrm{GeV} / \mathrm{c})^{2}$. The polarization then monotonically decreases to 0.0 at $\mathrm{t}=-4.03(\mathrm{GeV} / \mathrm{c})^{2}$. The polarization from $t=-.58$ to $t=-5.25(\mathrm{GeV} / \mathrm{c})^{2}$ was measured for $7.0 \mathrm{GeV} / \mathrm{c}$. From a minimum at $t=-.8(\mathrm{GeV} / \mathrm{c})^{2}$, the polarization rises to a maximum of .20 at $\mathrm{t}=-1.7(\mathrm{GeV} / \mathrm{c})^{2}$. From the starting point of $\mathrm{t}=-1.53(\mathrm{GeV} / \mathrm{c})^{2}$ the polarization rises to a maximum of .20 at $\mathrm{t}=-1.7(\mathrm{GeV} / \mathrm{c})^{2}$, then drops to a minimum at $t=-2.3(\mathrm{GeV} / \mathrm{c})^{2}$. There follows another maximum at $t=-2.9$ $(\mathrm{GeV} / \mathrm{c})^{2}$, then another minimum at $\mathrm{t}=-4.6(\mathrm{GeV} / \mathrm{c})^{2}$. The polarization is still rising for the last point measured at $t=-6.2(\mathrm{GeV} / \mathrm{c})^{2}$.

The results are discussed in terms of four models. Two of these are optical models; one was proposed by Durand and Lipes, the other by Chu and Hendry. The other two models are the Regge pole model and the parton model. Fits to the data were done using the first three models; the optical model of Chu and Hendry best described the structure in the data. 


\section{Table of Contents}

Abstract. ....................... . . i

Chapter I. Introduction .................. I

Chapter II. Theory .................... 4

Chapter III. Experiment . . . . . . . . . . . 18

Chapter IV. Analysis................ 43

Chapter V. Results and Conclusions... . . . . . . 67

List of Figure Captions. . . . . . . . . . . . 96

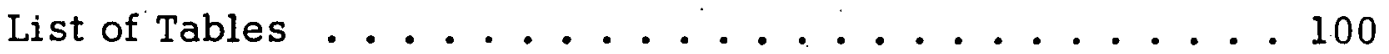




\section{Introduction}

Presented herein are the results of an experiment to measure the polarization in large angle proton-proton elastic scattering. This experiment was performed in the External Proton Beam I of the ZGS accelerator at Argonne National Laboratory using a primary beam whose intensity varied up to $10^{10}$ protons per burst. Large $|\mathrm{t}|$ values were examined with high statistics by utilizing, for the first time, a polarized proton target in a primary beam.. The momenta examined were $5.15,7.0$, and $12.33 \mathrm{GeV} / \mathrm{c}$; the center-of-mass angles covered for the first two momenta were $26^{\circ} \mathrm{s}$ ${ }^{\theta} \mathrm{C.m} . \leq 90^{\circ}$, and for the highest momentum $30^{\circ} \leq \theta_{\mathrm{C} . \mathrm{m} .} \leq 65^{\circ}$. This corresponded to a $t$ range $.5 \leq|t| \leq 6.3(\mathrm{GeV} / \mathrm{c})^{2}$.

The proton-proton system is one of the most basic systems for our understanding of the strong interaction. A complete description of the scattering of the two protons is given by a matrix which, given the initial spin and momentum, gives the final spin and momentum. Wolfenstein ${ }^{1}$, Goldberger $^{2}$, and other authors have shown that this matrix can be expressed in terms of five independent complex amplitudes. Thus, measurements of nine independent variables will determine the system up to an overall phase. These polarization data, which provide meaningful constraints over a large range of $t$, and certain assumptions about some of the amplitudes allow us to critically evaluate several current theories. Indeed, the polarization is very sensitive to any structure in these amplitudes as we will show later. Furthermore, those theories which survive these data 
should provide us with additional insight on the strong interaction.

Several years ago the proton-proton system did not appear to be very exciting. The cross section measurements were structureless, the polarization measurements were smooth. Recently structure has appeared. A dip ${ }^{3}$ has appeared in the differential cross section at $t=-1.0(\mathrm{GeV} / \mathrm{c})^{2}$ at high energies. A break ${ }^{4}$ in the $\theta_{\mathrm{c} . \mathrm{m} .}=90^{\circ}$ differential cross section has been found at $t=-6.5(\mathrm{GeV} / \mathrm{c})^{2}$. We now know that the $90^{\circ}$ differential cross section has slope changes ${ }^{5}$ at $t=-.8$ and $-2.5(\mathrm{GeV} / \mathrm{c})^{2}$. Evidence $^{6}$ for a slope change in the differential cross section near $t=-.1$ $(\mathrm{GeV} / \mathrm{c})^{2}$ has been seen for several energies. The once smooth polarization has a well established $\operatorname{dip}^{7,8}$ near $t=-.8(\mathrm{GeV} / \mathrm{c})^{2}$. We expect that this experiment will shed light on the correlations between the cross section structure and the polarization structure. 


\section{References}

1. L. Wolferistein and J. Ashkin, Phys. Rev. 15 , 947 (1952).

2. M. L. Goldberger, M. T. Grisaru, S. W. MacDowell, and D. Y. Wong, Phys. Rev. 120, 2250 (1960).

3. Aachen-CERN-Genova-Harvard-Turin Collaboration, Proceedings of the XVI International Conference on High Energy Physics (National Accelerator Laboratory, Batavia, 1972), Vol. 3.

4. C. W. Akerlof, R. H. Hieber, A. D. Krisch, K. W. Edwards, L. G. Ratner, and K. Ruddick, Phys. Rev. 159, 1138 (1967).

5. R. C. Kammerud, B. B. Brabson, R. R. Crittenden, R. M. Heinz, H. A. Neal, H. W. Paik, and R. A. Sidwell, Phys. Rev. D4, 1309 (1971).

6. G. Barbiellini, M. Bozzo, P. Darriulat, G. D. Palazzi, G. DeZorzi, A. Fainberg, M. I. Ferrero, M. Holder, A. McFarland, G. Maderni, S. Orito, J. Pilcher, C. Rubbia, A. Santroni, G. Sette, A. Straude, P. Strolin, and K. Tittel, Phys. Letters 39B, 663 (1972).

7. H. A. Neal and M. J. Longo, Phys. Rev. 161, 1374 (1967).

8. J. H. Parry, N. E. Booth, G. Conforto, R. J. Esterling, J. Scheid, and D..J. Sherden, Phys. Rev. D8, 45 (1973). 


\section{Theory}

A. Formalism of Proton-Proton Scattering

Wolfenstein ${ }^{l}$ has given a clear derivation of the scattering matrix, $M$, based on the physical properties of the proton-proton system. For two spin 1/2 particles the scattering matrix is an operator in the fourdimensional spin space. Given the initial spin and momentum state, $M$ determines the final spin and momentum state.

Let

$\sigma_{1}=\operatorname{spin}$ operator of one particle

$\underline{\sigma}_{2}=$ spin operator of the other particle

$\underline{k}=$ initial momentum of one particle in the center-of-mass (c.m.) frame

$\underline{k}^{\prime}=$ final $c \cdot m:$ momentum of the same particle.

Define

$$
\begin{aligned}
& \underline{\mathrm{K}}=\underline{\mathrm{k}}-\underline{\mathrm{k}}^{\prime} \\
& \underline{\mathrm{n}}=\underline{\mathrm{k}} \times \underline{\mathrm{k}}^{\prime} \\
& \underline{\mathrm{p}}=\underline{\mathrm{n}} \times \underline{\mathrm{k}}
\end{aligned}
$$

Since $M$ is a $4 \times 4$ matrix, it is composed of 16 linearly independent matrices in spin space, all of which are functions of 1 (unit matrix), $\underline{\sigma}_{1}$ ' and $y_{2}$. These are: 


$\begin{array}{ccc}\text { Combination } & \text { Type } & \begin{array}{c}\text { Number of linearly } \\ \text { independent matrices }\end{array} \\ \underline{\sigma}_{1} \cdot \underline{\sigma}_{2}-1 & \text { scalar } & 1 \\ \underline{\sigma}_{1}+\underline{\sigma}_{2} & \text { scalar } & 3 \\ \underline{\sigma}_{1}-\underline{\sigma}_{2} & \text { vector } & 3 \\ \underline{\sigma}_{1} \times \underline{\sigma}_{2} & \text { vector } \\ \sigma_{1 \alpha} \sigma_{2 \beta}+\sigma & \text { vector } & 3 \\ 1 \sigma_{2 \alpha}^{\sigma}=\mathrm{t}_{\alpha \beta} & \text { tensor } & 5\end{array}$

where $\alpha, \beta$ denote the $x, y, z$ components of the vector.

These linearly independent matrices may be combined with any of the following combinations of momenta:

$$
\begin{array}{ll}
\text { Scalars } & 1, \underline{k} \cdot \underline{k^{\prime}}, k^{2}=k^{\prime 2} \\
\text { Vectors } & \underline{K}, \underline{n}, \underline{P} \\
\text { Tensors } & K_{\alpha} K_{\beta}, n_{\alpha} n_{\beta}, P_{\alpha} P_{\beta}, K_{\alpha} P_{\beta}, n_{\alpha} P_{\beta}, n_{\alpha} K_{\beta}, K_{\alpha} P_{\beta} \pm K_{\beta} P_{\alpha} \\
& n_{\alpha} K_{\beta} \pm K_{\alpha} n_{\beta}, n_{\alpha} P_{\beta} \pm P_{\alpha \beta} n
\end{array}
$$

The conditions placed on $\mathrm{M}$ are invariance under space rotations and reflections and invariance under time reversal.

$$
\text { Under space reflections } \underline{\sigma}_{1} \rightarrow \underline{\sigma}_{1}: \underline{\sigma}_{2} \rightarrow \underline{\sigma}_{2}, \underline{\mathrm{k}} \rightarrow-\underline{\mathrm{k}} \text {, and } \underline{\mathrm{k}}^{\prime} \rightarrow-\underline{\mathrm{k}}^{\prime} \cdot \text { Ail }
$$

16 matrices satisfy this condition. The functions of momenta which do are $\underline{n}$ and $n_{\alpha} n_{\beta}$. Also satisfying this condition are dot products and tensors using $\underline{K}$ and $\underline{P}$; these are $k^{2}, k^{\prime 2}, P_{\alpha} P_{\beta}, K_{\alpha} P_{\beta}$, and $K_{\alpha} P_{\beta} \pm K_{\beta} P_{\alpha}$.

Combining them we get these possibilities: $1,\left(\underline{\sigma}_{1} \cdot \underline{o}_{2}-1\right),\left(\underline{\sigma}_{1}+\underline{\sigma}_{2}\right)$ - In, $\left(\sigma_{1}-\sigma_{2}\right) \cdot \underline{n},\left(\underline{\sigma}_{1} \times \underline{\sigma}_{2}\right) \cdot \underline{n}, \Gamma_{\alpha \beta} t_{\alpha \beta} T_{\alpha \beta}$ where $T_{\alpha \beta}$ is any one of the 
tensor functions of momenta. Any of the above may be multiplied by functions of $\mathrm{k}^{2}$ and $\underline{\mathrm{k}} \cdot \underline{\mathrm{k}}^{\prime}$.

Under time reversal $\underline{\sigma}_{1} \rightarrow-\underline{\sigma}_{1}, \underline{\sigma}_{2} \rightarrow-\underline{\sigma}_{2}, \underline{k} \rightarrow-\underline{k}$, and $\underline{k}^{\prime} \rightarrow-\underline{k}^{\prime}$, so $\underline{K} \rightarrow \underline{K}, \underline{n} \rightarrow-\underline{n}$, and $\underline{\underline{p}} \rightarrow-\underline{p}$. Now time reversal makes $1,\left(\underline{\sigma}_{1} \cdot \underline{\sigma}_{2}-1\right)$ and $\left(\underline{\sigma}_{1}+\underline{\sigma}_{2}\right) \cdot \underline{\mathrm{n}}$ stay constant, but $\left(\underline{\sigma}_{1}-\underline{\sigma}_{2}\right) \cdot \mathrm{n}$ and $\left(\underline{\sigma}_{1} \times \underline{\sigma}_{2}\right) \cdot \underline{\mathrm{n}}$ change sign; of the matrix tensors $t_{\alpha \beta} \rightarrow_{{ }_{\alpha \beta}}$, so we can only use the momentum tensors that do not change sign; these are $K_{\alpha} K_{\beta^{\prime}} n_{\alpha} n_{\beta}$, and $P_{\alpha} P_{\beta}$. In vector notation these are

$$
\begin{aligned}
& \sum_{\alpha \beta} \mathrm{t}_{\alpha \beta} \mathrm{K}_{\alpha} \mathrm{K}_{\beta}=\left(\underline{\sigma}_{1} \cdot \underline{\mathrm{K}}\right)\left(\underline{\sigma}_{2} \cdot \underline{\mathrm{K}}\right) \\
& \sum_{\alpha \beta} t_{\alpha \beta^{n} \alpha^{n} \cdot \beta}=\left(\underline{\sigma}_{1} \cdot \underline{n}\right)\left(\underline{\sigma}_{2} \cdot \underline{n}\right) \\
& \sum_{\alpha \beta} \mathrm{t}_{\alpha \beta^{2}} \mathrm{P}_{\alpha \beta} \mathrm{P}_{\beta}=\left(\underline{\sigma}_{1} \cdot \underline{\mathrm{P}}\right)\left(\underline{\sigma}_{2} \cdot \underline{\mathrm{P}}\right)
\end{aligned}
$$

But only two of these are independent since they can be combined to form $\underline{\sigma}_{1} \cdot \underline{\sigma}_{2} \cdot$

Thus the general form of $M$ is

$$
\begin{aligned}
\mathrm{M}= & \mathrm{A}+\mathrm{B}\left(\underline{\sigma}_{1} \cdot \underline{\sigma}_{2}-1\right)+\mathrm{C}\left(\underline{\sigma}_{1}+\underline{\sigma}_{2}\right) \cdot \underline{\mathrm{n}}+\mathrm{D} \underline{\sigma}_{1} \cdot \underline{\mathrm{K}}_{2} \\
& \cdot \underline{\mathrm{K}}+\mathrm{E} \underline{\sigma}_{1} \cdot \underline{\mathrm{g}}_{2} \cdot \underline{\mathrm{\sigma}}+\mathrm{F}\left(\underline{\sigma}_{1}-\underline{\sigma}_{2}\right) \cdot \underline{\mathrm{n}}
\end{aligned}
$$

Now if both spin $1 / 2$ particles are protons then $M$ must be invariant under interchange of $\underline{\sigma}_{1}$ and $\underline{\sigma}_{2}$ because of identical particles. So $F\left(\underline{\sigma}_{1}-\underline{\sigma}_{2}\right) \cdot n$ goes to $F\left(\underline{\sigma}_{2}-\underline{\sigma}_{1}\right) \cdot \underline{n}$ which implies $F=0$. Then $M$ has five unknowns which are complex functions of $k^{2}$ and $\underline{k} \cdot \underline{k}^{\prime}$. 
B. Helicity Formalism

The helicity formalism as developed by Jacob and Wick ${ }^{9}$ has an intrinsic simplicity that facilitates the discussion of proton-proton scattering.

Define these quantities:

$$
\begin{aligned}
& M=\text { scattering matrix } \\
& T=\text { transition matrix } \\
& \lambda_{1}=\text { helicity of one incident proton } \\
& \lambda_{2}=\text { helicity of the other incident proton } \\
& \lambda_{1}^{\prime}=\text { helicity of one final proton } \\
& \lambda_{2}^{\prime}=\text { helicity of the other final proton } \\
& \lambda^{\prime}=\lambda_{1}-\lambda_{2} \\
& \lambda^{\prime}=\lambda_{1}^{\prime}-\lambda_{2} \\
& d^{J} \lambda^{\prime}(\theta)=\text { reduced rotation matrix } \\
& \theta^{\prime}=\mathrm{c} \cdot \mathrm{m} . \text { scattering angle } \\
& \mathrm{k}=\mathrm{c} \cdot \mathrm{m} . \text { momentum }=|\underline{\mathrm{k}}|=\left|\underline{\mathrm{k}^{\prime}}\right| \\
& \mathrm{W}=\text { total c.m. energy. }
\end{aligned}
$$

Jacob and $W^{\prime}$ ick have made a partial wave decomposition of the scattering amplitude in terms of the Lorentz invariant transition amplitudes. This expansion is

$$
\begin{aligned}
\left\langle\lambda_{1}^{i} \lambda_{2} \cdot|\mathrm{M}| \lambda_{1} \lambda_{2}\right\rangle= & \frac{1}{\mathrm{k}} \sum_{\mathrm{J}}(2 \mathrm{~J}+1)\left\langle\lambda_{1} \lambda_{2}\left|\mathrm{~T}^{\mathrm{J}}(\mathrm{W})\right|\right. \\
& \left.\lambda_{1} \lambda_{2}\right\rangle \mathrm{d}^{\mathrm{J}}{ }_{\lambda \lambda^{\prime}}(\theta)
\end{aligned}
$$


Let us choose the following as helicity basis states:

$$
\begin{aligned}
& \left|\psi_{1}\right\rangle=\left|\lambda_{1}=+1 / 2, \lambda_{2}=+1 / 2\right\rangle=|++\rangle \\
& \left|\psi_{2}\right\rangle=|+1 / 2-1 / 2\rangle=|+-\rangle \\
& \left|\psi_{3}\right\rangle=|-1 / 2+1 / 2\rangle=|-+\rangle \\
& \left|\psi_{4}\right\rangle=|-1 / 2-1 / 2\rangle=|--\rangle
\end{aligned}
$$

So a matrix element will be

$$
M_{j i}=\left\langle\psi_{j}|M| \psi_{i}\right\rangle
$$

Goldberger ${ }^{2}$ has selected five of these amplitudes as the independent ones

$$
\begin{aligned}
& \Phi_{1}=M_{11}=\langle+|M|++\rangle \\
& \Phi_{2}=M_{14}=\langle++|M|-\rangle \\
& \Phi_{3}=M_{22}=\langle+-|M|+-\rangle \\
& \Phi_{4}=M_{23}=\langle+-|M|-+\rangle \\
& \Phi_{5}=M_{12}=\langle++|M|+\rangle
\end{aligned}
$$

In terms of these five amplitudes $M$ is

$$
M=\left|\begin{array}{cccc}
\Phi_{1} & \Phi_{5} & -\Phi 5 & \Phi_{2} \\
-\Phi & \Phi_{3} & \Phi_{4} & -\Phi 5 \\
{ }^{5} & \Phi_{4} & \Phi_{3} & \Phi_{5} \\
5 & & & \\
\Phi_{2} & \Phi_{5} & -\Phi_{5} & \Phi_{1}
\end{array}\right|
$$




\section{Definition of Polarization}

We present two equivalent definitions of polarization. One in terms - of helicity amplitudes will be used in the theoretical interpretation of the data. The other is a working definition for experimental use.

Define ${ }^{l} \rho$ to be

$$
\rho=\sum_{n} P_{n} X_{n} X_{n}^{+}
$$

where $X_{n}$ is a 4-component column vector representing a pure spin state, $\mathrm{X}_{\mathrm{n}}^{+}$is the adjoint row vector, $\dot{\mathrm{P}}_{\mathrm{n}}$ is the relative probability of finding the system in the $X_{n}$ state.

Given any spin operntor 0 the average valuc of 0 is defined by

$$
\langle 0\rangle \operatorname{tr} \rho=\operatorname{tr}(\rho 0)
$$

The density matrix, $\rho$, is $4 \times 4$ and Hermitian. It is spanned by a complete set of 16 Hermitian operators $S^{\lambda}$ where complete means

$$
\operatorname{tr} S^{\lambda} S^{\nu}=4 \delta^{\lambda \nu}
$$

For example the set composed of $1, \underline{\sigma}_{1}, \underline{\sigma}_{2}$ (Paull matrices), and products of $\sigma_{1 \mathrm{r}}$ and $\sigma_{2 s}, r=x, y, z, s=x, y, z$, form a complete set. Thus $p$ can be expanded in torms of such a set

$$
\rho=\sum_{\lambda} a^{\lambda} s^{\lambda}
$$

To solve for $\mathrm{a}^{\lambda}$ multiply both sides of eq. 8 by $\mathrm{S}^{\nu}$ and take the trace

$$
\operatorname{tr} \rho S^{\nu}=\Sigma a^{\lambda} \cdot \operatorname{tr} S^{\nu} S^{\lambda}
$$


Now using equation 7 we get

$$
a^{\lambda}=1 / 4 \operatorname{tr} \rho s^{\lambda}
$$

So

$$
\rho=1 / 4 \Sigma \operatorname{tr}\left(\rho S^{\lambda}\right) S^{\lambda}=1 / 4 \operatorname{tr} \rho \Sigma_{\lambda}\left\langle S^{\lambda}\right\rangle S^{\lambda}
$$

by eq. 6 .

Now if we let $\rho_{i}$ and $\rho_{f}$ be the density matrix before and after scattering, then by the definition of $M$

$$
\rho_{f}=M \rho_{i} M^{+}
$$

Now suppose the density matrix $\rho_{i}$ is of an unpolarized beam on an unpolarized target, then $\rho_{i}=1 / 41$ since all $\left\langle S^{\lambda}\right\rangle=0$ except for the unit matrix (this implies tr $p_{i}=1$ ). In this case the differential cross section is defined by

$$
\begin{aligned}
& I_{O}=\operatorname{tr} \rho_{f}=1 / 4 \operatorname{tr} M^{+} \\
& =1 / 4 \operatorname{tr}\left|\begin{array}{cccc}
\Phi_{1} & \Phi_{5} & -\Phi_{5} & \Phi_{2} \\
-\Phi_{5} & \Phi_{3} & \Phi_{4} & -\Phi_{5} \\
\Phi_{5} & \Phi_{4} & \Phi_{3} & \Phi_{5} \\
\Phi_{2} & \Phi_{5} & -\Phi_{5} & \Phi_{1}
\end{array}\right| \quad\left|\begin{array}{cccc}
\Phi_{1}{ }^{*} & -\Phi_{5} * & \Phi_{5}{ }^{*} & \Phi_{2} \\
\Phi_{5}{ }^{*} & \Phi_{3} * & \Phi_{4}{ }^{*} & \Phi_{5}{ }^{*} \\
-\Phi_{5} & \Phi_{4}{ }^{*} & \Phi_{3}{ }^{*} & -\Phi_{5} * \\
\Phi_{2} & -\Phi_{5} * & \Phi_{5}{ }^{*} & \Phi_{1} \star
\end{array}\right| \\
& =1 / 2\left(\left|\Phi_{1}\right|^{2}+\left|\Phi_{2}\right|^{2}+\left|\Phi_{3}\right|^{2}+\left|\Phi_{4}\right|^{2}+4\left|\Phi_{5}\right|^{2}\right)
\end{aligned}
$$

after multiplication of the matrices and reduction of the trace to the simpliest form.

Now suppose the target is polarized to $\mathrm{P}_{\mathrm{T}}$ in the $\mathrm{y}$-direction, then 


$$
\rho_{\mathrm{i}}=1 / 4\left[\left|\begin{array}{ll}
\mathrm{P}_{\mathrm{T}} \sigma_{\mathrm{ly}} & 0 \\
0 & \mathrm{P}_{\mathrm{T}} \sigma_{\mathrm{ly}}
\end{array}\right|+1\right]
$$

where $\sigma_{\text {ly }}$ is the 2-dimensional Pauli matrix.

Then

$$
I=\operatorname{tr} \rho_{f}=\operatorname{tr} M \rho_{i} M^{+}
$$

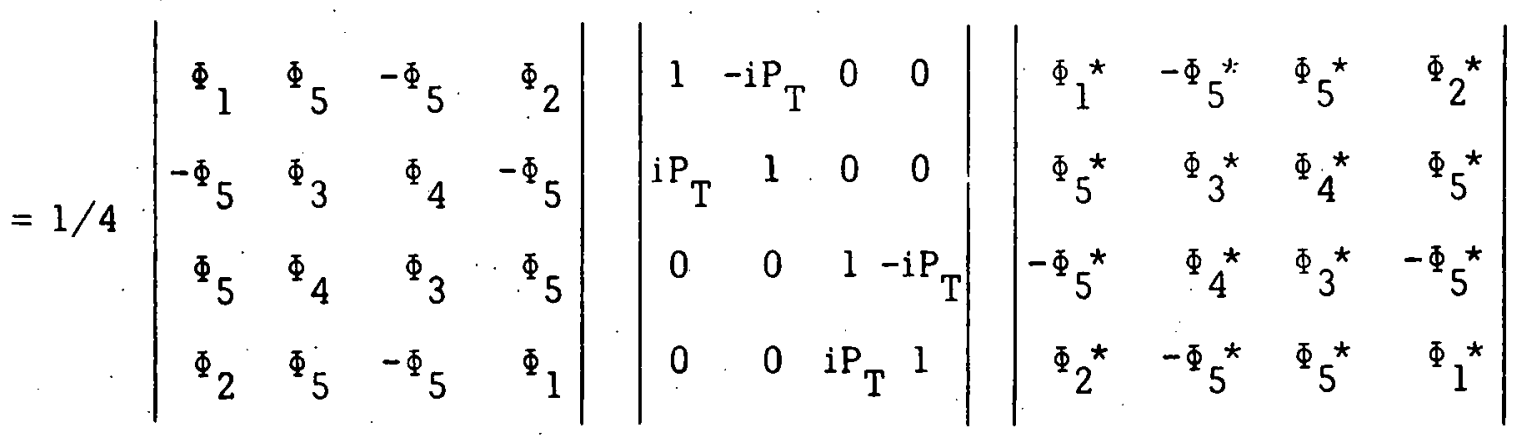

$=I_{0}-P_{T} \operatorname{Im}\left[\left(\Phi_{1}+\Phi_{2}+\Phi_{3}-\Phi_{4}\right) \Phi_{5}{ }^{\star}\right]$

$=\mathrm{I}_{\mathrm{O}}\left(\mathrm{l}+\mathrm{P}_{\mathrm{T}} \mathrm{P}\right)$

where

$$
P=-\operatorname{Im}\left[\left(\Phi_{1}+\Phi_{2}+\Phi_{3}-\Phi_{4}\right) \Phi_{5}^{*}\right] / I_{0}
$$

Now suppose we have $\mathrm{P}_{\mathrm{T}}>0$ (spin up)

then

$$
I_{u}=I_{0}\left(1+P_{T u}{ }^{P}\right)\left(P_{T u}>0\right)
$$

and for

$$
\begin{aligned}
& \mathrm{P}_{\mathrm{T}}<0 \quad \mathrm{P}_{\mathrm{T}}=-\mathrm{P}_{\mathrm{Td}}\left(\mathrm{P}_{\mathrm{Td}}>0\right) \\
& \mathrm{I}_{\mathrm{d}}=\mathrm{I}_{\mathrm{O}}\left(1-\mathrm{P}_{\mathrm{Td}} \mathrm{P}\right)
\end{aligned}
$$

solving for $P$ we get

$$
I_{u}\left(1-P_{T d} P\right)=I_{d}\left(1+P_{1} P u^{P}\right.
$$




$$
P=\frac{1}{\mid\left(P_{T}\right)} \mid \quad \frac{I_{u}-I_{d}}{I_{u}+I_{d}}
$$

where we have assumed $P_{T u}=P_{T d}=\left|P_{T}\right|$. Here $I_{u}$ (and $I_{d}$ ) represent the number of counts per unit beam. So eq'. 13 represents a working definition of the polarization.

\section{Models of Proton-Proton Scattering}

In the section on results the polarization at the three momenta will be discussed in terms of four models. In this section we would like to give a brief explanation of these models.

The Regge pole model ${ }^{10}$ presents the scattering matrix as an expansion in total angular momentum

$$
M(x, t)=\sum_{J=0}^{\infty} P_{J}(x) A(J, t)
$$

where $x=\cos \theta$

$\mathrm{t}=$ square of the four momentum transfer squared

then extends $M$ to complex $J$, that is, analytically continues $M$ to the complex J plane. Let $\alpha(t)$ represent the continued J, then

$$
M(x, t)=\frac{1}{2 \pi i} \oint d \alpha \frac{\pi}{\sin \pi \alpha} P_{\alpha}(-x) A(\alpha, t)
$$

where $\pi / \sin \pi \alpha$ gives the residues $(-1)^{J}\left(\right.$ recall $\left.(-1)^{J} P_{J}(x)=P_{J}(-x)\right)$. The integral is around the real line; if we distort the path to $-1 / 2-i \infty$ to $-1 / 2+i \infty$, then

$$
M(x, t)=-\frac{1}{2 \pi} \int_{-1 / 2-i \infty}^{-1 / 2+i \infty} d \alpha \frac{P_{\alpha}(-x) A(\alpha, t)}{\sin \pi \alpha}+\sum_{i} \beta_{i}(t) \frac{P_{\alpha_{i}}(-x)}{\sin \pi \alpha_{i}}
$$


where $\alpha_{i}(t)$ represent the poles in the $\alpha$ plane and $\beta_{i}(t)$ are the residues of the poles. The poles are called Regge poles and are associated with particles or particle resonances.

The next model is an optical model proposed by Chu and Hendry ${ }^{9}$. The proton-proton s-channel partial-wave helicity amplitudes are represented as functions of the impact parameter $b$. They are composed of two parts, a diffraction piece and a peripheral piece.

We know that (see equation 12)

$$
P \sim-\operatorname{Im}\left[\Phi_{5}^{*}\left(\Phi_{1}+\Phi_{2}+\Phi_{3}-\Phi_{4}\right)\right]
$$

Hendry and Chu ${ }^{11}$ assume that $\Phi_{2}$ and $\Phi_{4}$ are insignificant compared to $\Phi_{1}$ and $\Phi_{3}$ and so are taken to be $0 . \Phi_{1}$ and $\Phi_{3}$, the helicity non-flip amplitudes, arise from both a diffractive and a peripheral piece. In the s-channel the non-flip amplitude is given by

$$
M_{++}^{s}=\frac{1}{k} \sum_{J}(J+1 / 2) T_{++}^{J} d_{\frac{1}{2} \frac{1}{2}}^{J}(\theta)
$$

Now for diffraction let us assume that in a first approximation

$$
\begin{array}{rlrl}
\mathrm{T}_{++}^{\mathrm{J}} & =\mathrm{i} \mathrm{a}_{1} & \mathrm{~J} \leq \mathrm{kR}=\mathrm{J}_{\max } \\
& =0 & \mathrm{~J}>\mathrm{kR} \\
\mathrm{M}_{++}^{\mathrm{s}}=\frac{\mathrm{i} \mathrm{a}_{1}}{\mathrm{k}} \sum^{\mathrm{J}_{\max }}(\mathrm{J}+\mathrm{l} / 2) \mathrm{d}_{\frac{\mathrm{l}}{2}}^{\mathrm{J}} \frac{\mathrm{l}}{2}
\end{array}
$$

We would like to express $M_{++}^{s}$ as an integral. We have

$$
\mathrm{d}_{\frac{1}{2} \frac{1}{2}}^{J}(\theta) \approx J_{0}((2 J+1) \sin \theta / 2) .
$$

Let $(J+1 / 2)=k b$, then $(2 J+1) \sin \theta / 2=(2 k \sin \theta / 2) b=\sqrt{-t} b$ 
This means that

$$
M_{++}^{S} \rightarrow \frac{i a_{1}}{k} \int_{0}^{R} k b k d b J_{0}(\sqrt{-t} b)=i a_{1}^{\prime} \frac{J_{1}(\sqrt{-t} R)}{\sqrt{-t} R}
$$

For the peripheral piece let

$$
\mathrm{T}_{++}^{\mathrm{J}}=\mathrm{a}_{2} \delta(\mathrm{b}-\mathrm{R})
$$

Immediately we get this part proportional to $J_{0}(\sqrt{-t} R)$. Adding the two parts makes

$$
M_{++}^{s} \sim i a_{1}^{\prime} \frac{J_{1}(\sqrt{-t} R)}{\sqrt{-t} R}+a_{2}^{\prime} J_{0}(\sqrt{-t} R)
$$

For the helicity flip amplitude we have

$$
M_{+-}^{s}=\frac{1}{k} \Sigma(J+1 / 2) T_{+-\frac{1}{2} \frac{1}{2}}^{J}(\theta)
$$

But

$$
\mathrm{d}_{-\frac{1}{2} \frac{1}{2}}^{\mathrm{J}} \approx \mathrm{J}_{1}((2 \mathrm{~J}+1) \sin \theta / 2)=J_{1}(\sqrt{-\mathrm{t}} \mathrm{b})
$$

if $k b=J+i / 2$. Assume ${ }^{12}$ the flip amplitude has only a peripheral piece so

$$
\mathrm{T}_{+-}^{\mathrm{J}}=\mathrm{b}_{1} \delta \quad(\mathrm{b}-\mathrm{R})
$$

giving

$$
\dot{M}_{+-}^{S} \approx b_{1} J_{1}(\sqrt{-t} R), b_{1} \text { real }
$$

Then the polarization

$$
P \sim \frac{J_{1}(\sqrt{-t} R)}{\sqrt{-t} R} \quad J_{1}(\sqrt{-t} R)
$$

This form for the polarization exhibits ${ }^{13}$ a single zero at $-t=0$ and double zeros at each zero of the $J_{1}$ Bessel function. 
In reality the model is much more sophisticated than presented. The diffraction piece ${ }^{14}$ is really represented as

$$
\mathrm{T}_{++}^{\mathrm{J}}=\mathrm{F}^{\mathrm{r}}(\mathrm{b})+i \mathrm{~F}^{\mathrm{i}}(\mathrm{b})
$$

where the Fermi distribution

$$
F(b)=f_{1} /\left\{1+\exp \left(f_{2}\left[b-f_{3}\right]\right)\right\}
$$

$\mathrm{f}_{3}$ is a radius term

$\mathrm{f}_{2}$ determines the inverse surface thickness

$f_{1}$ is the strength

The peripheral piece is represented by a Gaussian

$$
\mathrm{T}_{+-}^{\mathrm{J}}=\mathrm{g}_{1} \exp \left[-\mathrm{g}_{2}\left(\mathrm{~b}-\mathrm{g}_{3}\right)^{2}\right]
$$

Durand, Lipes ${ }^{15}$, Chou, Yang ${ }^{16,17}$, and others ${ }^{18}$ have proposed similar diffraction scattering models for $p-p$ scattering. In particular, the DurandLipes model makes two assumptions. High energy elastic scattering of hadrons is primarily diffractive. The incident wave function is absorbed into many inelastic channels; this absorption is proportional to the total amount of interpenetrating hadronic matter for a given impact parameter .

These assumptions imply that the $S$ scattering matrix $(S=1+i M)$ is

$$
S(b)=\exp (-x \rho(b)) \quad(x \text { is a constant })
$$

where $\rho(b)$ is given in the equation

$$
G_{A}(t) \cdot G_{B}(t)=\int_{0}^{\infty} p(b) \cdot J_{0}(b \sqrt{-t}) b d b
$$

$G_{A}(t)$ and $G_{B}(t)$ are matter form factors for hadrons $A$ and $B$. Durand and Lipes use the proton charge form factor as an approximation for the matter 
form factor.

Some of the predictions of these models are as follows:

(a) The asymptotic $p-p$ scattering cross section should display two diffraction minima which will begin to appear at nonasymptotic energies. These are $s$ independent for large $s$ and will appear near $-t=1-2(\mathrm{GeV} / \mathrm{c})^{2}$ and $-\mathrm{t}=5-8(\mathrm{GeV} / \mathrm{c})^{2}$.

(b) At very large - $t$, the scattering amplitude should approach the product of form factors.

(c) Near the first minimum, the $p-p$ scattering should have a large real part.

(d) The proton polarization should have two zeros in the region $-t \approx 1-2(\mathrm{GeV} / \mathrm{C})^{2}$ that could appear at the same place. 19

In the parton model. 20 physical particle is composed of pointlike particles, called partons. Partons are quanta of $\mathrm{H}_{\mathrm{O}^{\prime}}$, the kinetic energy portion of the Hamiltonian. Hadrons are assumed to be collections of partons. The probability of finding a hadronic parton in some region of momentum space is assumed to be approximately constant per unit $\ln P_{\ell}$ (the component of the particle's momentum parallel to the incident momentum) and rapidly decreasing as $\mathrm{P}_{\mathrm{T}}$ (the transverse momentum) increases. The parton distributions of both the target and projectile are modified in a collision. The model assumes that the simpliest elementary process is high $-\mathrm{P}_{\mathrm{T}}$ parton-parton scattering through the exchange of a single parton. Specifically, the parton quark ${ }^{21}$ model predicts that for high energies and large scattering angles, the scattering amplitude is purely real in $p-p$ scattering, leading to no polarization. 


\section{References}

9. M. Jacob and G. C. Wick, Ann. Phys. Z, 404 (1959).

10. H. Muirhead, The Physics of Elementary Particles (Pergamon Press, Oxford, 1965), Sec. 10.5.

11. T-Y Cheng, S-Y Chu, and A. W. Hendry, Phys. Rev. D7, 86 (1973).

12. S-Y Chu and A. W. Hendry, Phys. Rev. D6; 190 (1972).

13. A. W. Hendry, private communication.

14. S-Y Chu and A. W. Hendry, Phys. Rev. D4, 3282 (1971).

15. L. Durand and R. Lipes, Phys. Rev. Letters $\underline{20}, 637$ (1968).

16. T. T. Chou and C. N. Yang, Phys. Rev. 170,1591 (1968).

17. T. T. Chou and C. N. Yang, Phys: Rev. Letters 20, 1213 (1968).

18. H. D. I. Abarbanel, S. D. Drell, and F. J. Gilman, Phys. Rev. Letters $\underline{20}, 280$ (1968).

19. L. Durand, private communication.

20. S. M. Berman, J. D. Bjorken, and J. B. Kogut, Phys. Rev. D4, 3388 (1971).

21. J. F. Gunion, S. J. Brodsky, and R. Blankenbecler, Phys. Letters $\underline{39 B}$, 649 (1972). 
III. Experiment

A. Equipment

The layout of the experimental apparatus is shown in Fig. 1. A beam of $10^{9}-10^{10}$ protons per burst was split off the ZGS External Proton Beam I, transmitted through a dispersive optics system, and then directed onto a two inch long ethylene glycol polarized proton target which was located in a $25 \mathrm{kG}$ magnetic field. The scattered protons were detected in a two arm multiwire proportional chamber spectrometer. Each arm was motor driven and its angular position was remotely controlled. The position of each arm was determined by a measuring system consisting of floor mounted measuring tapes, at both the front and the back of each arm, that were viewed by a TV camera looking through a cross hair connected to the arm. The TV signals were multiplexed through one monitor located at the romote controls for the arms in the electronics trailer.

The upstream side of each spectrometer arm had a trigger counter, a three element hodoscope, four multiwire proportional chambers used to define the scattering angle, and another trigger counter. These proportional chambers defined a vector into the spectrometer magnet located in the middle of the arm. After the magnet there was another trigger counter, four more proportional chambers that defined the vector out of the magnet, the final trigger counter, and another three element hodoscope. All the positions of the chambers and counters are shown in Table 1. Looking downstream, the arm on the left was called the fast arm, on the right the 


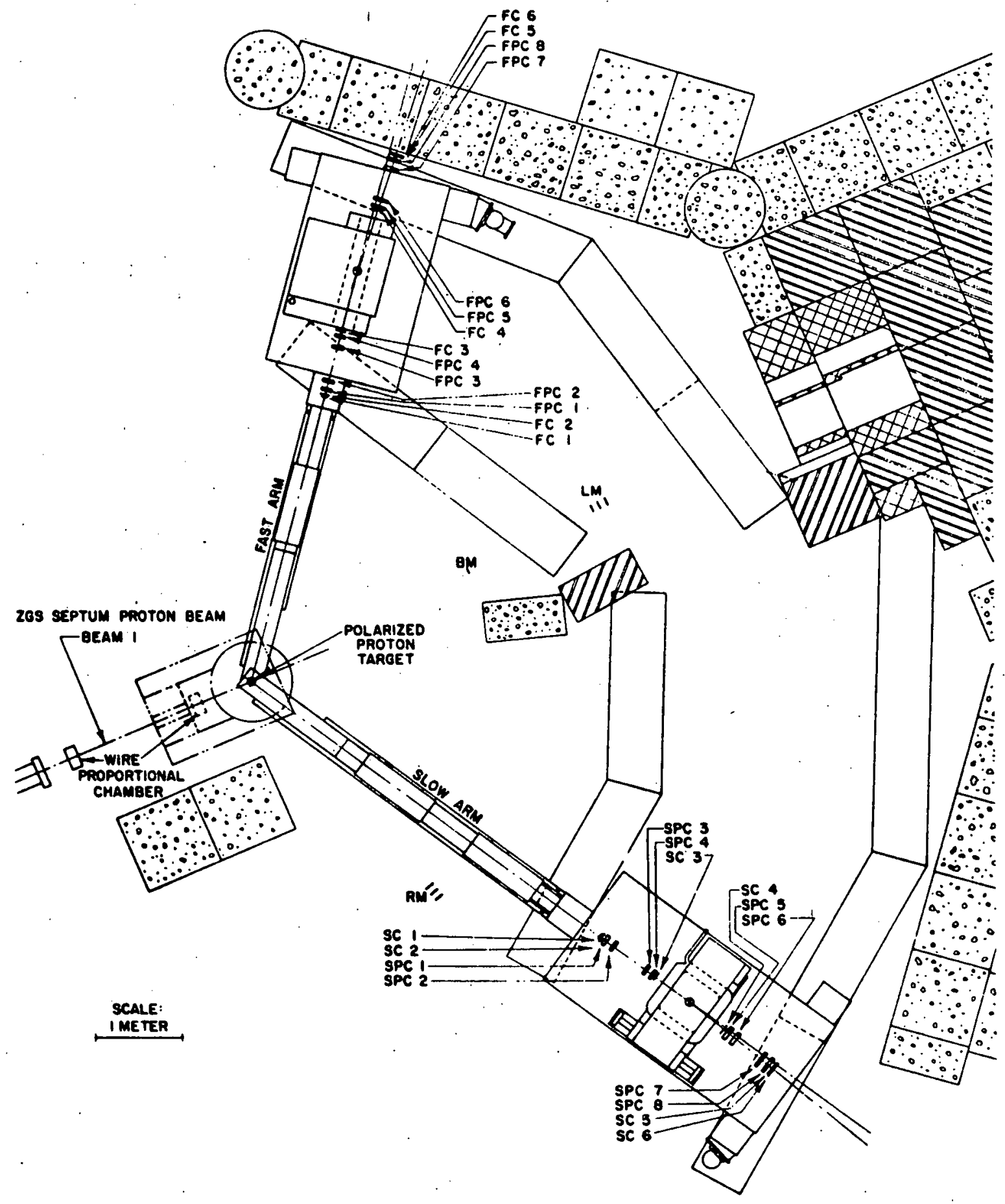

FIGURE 1 
TABLE 1

COUNTER AND CHAMBER POSITIONS

FAST ARM

\begin{tabular}{|c|c|c|c|c|}
\hline Part & Location $^{(\mathrm{a})}$ & & & Size \\
\hline $\mathrm{FCl}^{(\mathrm{c})}$ & $\mathrm{Z}=131.43^{(\mathrm{b})}$ & $-1.40 \leq X \leq 1.40$ & $-1.40 \leq Y \leq 1.40$ & $2.80 \times 2.80$ \\
\hline $\begin{array}{l}\text { FC2 } \\
\text { (hodoscope) }\end{array}$ & $Z=132.43$ & $-1.75 \leq X \leq 1.75$ & $-1.75 \leq Y \leq 1.75$ & $3.50 \times 3.50$ \\
\hline $\mathrm{FPCl}^{(\mathrm{d})}$ & $Z=134.53$ & $-2.22 \leq X \leq 2.42$ & $-2.35 \leq \mathrm{Y} \leq 2.35$ & $4.60 \times 4.60$ \\
\hline $\mathrm{FPC} 2$ & $Z=139.53$ & $-2.31 \leq x \leq 2.33$ & $-2.35 \leq Y \leq 2.35$ & $4.60 \times 4.60$ \\
\hline FPC3 & $Z=155.63$ & $-2.27 \leq x \leq 2.37$ & $-2.35 \leq Y \leq 2.35$ & $4.60 \times 4.60$ \\
\hline FPC 4 & $Z=160.63$ & $-2.35 \leq X \leq 2.26$ & $-2.35 \leq Y \leq 2.35$ & $4.60 \times 4.60$ \\
\hline FC3 & $z=163.33$ & $-1.97 \leq X \leq 2.03$ & $-2.00 \leq Y \leq 2.00$ & $4.00 \times 4.00$ \\
\hline
\end{tabular}

The remaining parts in the fast arm have $\mathrm{Z}$-axis rotated $2.464^{\circ}$ clockwise. $\mathrm{Z}^{\mathrm{l}}$ is the measurement from the center of the fast-arm magnet to the part. The magnet center has unrotated $Z=191.15625$.

\begin{tabular}{l|l|l|l|l|l}
\hline FC4 & $Z^{1}=29.50$ & $-2.50 \leq X^{1} \leq 2.50$ & $-2.50 \leq Y^{1} \leq 2.50$ & $5.00 \times 5.00$ \\
FPC5 & $Z^{1}=31.06$ & $-3.56 \leq X^{1} \leq 3.43$ & $-3.89 \leq Y^{1} \leq 3.12$ & $7.00 \times 7.00$ \\
FPC6 & $Z^{1}=35.06$ & $-3.53 \leq X^{1} \leq 3.46$ & $-3.20 \leq Y^{1} \leq 3.80$ & $7.00 \times 7.00$ \\
FPC7 & $Z^{1}=49.34$ & $-3.48 \leq X^{1} \leq 3.51$ & $-3.11 \leq Y^{1} \leq 3.89$ & $7.00 \times 7.00$ \\
FPC8 & $Z^{1}=53.34$ & $-3.53 \leq X^{1} \leq 3.45$ & $-3.85 \leq Y^{1} \leq 3.15$ & $7.00 \times 7.00$ \\
FC5 & $Z^{1}=55.70$ & $-3.26 \leq X^{1} \leq 3.24$ & $-2.75 \leq Y^{1} \leq 2.75$ & $6.50 \times 6.50$ \\
FC6 & $Z^{l}=56.00$ & $-3.25 \leq X^{1} \leq 3.25$ & $-2.75 \leq Y^{1} \leq 2.75$ & $6.50 \times 6.50$ \\
(hodoscope) & &
\end{tabular}


SLOW ARM

\begin{tabular}{|c|c|c|c|c|}
\hline Part & Location $^{(\mathrm{a})}$ & & & Size \\
\hline $\mathrm{SCl}{ }^{(\mathrm{c})}$ & $Z=193.60^{(b)}$ & $-1.50 \leq X \leq 1.50$ & $-1.50 \leq Y \leq 1.50$ & $3.00 \times 3.00$ \\
\hline $\begin{array}{l}\mathrm{SC} 2 \\
\text { (hodoscope) }\end{array}$ & $Z=194.30$ & $-1.75 \leq X \leq 1.75$ & $-1.75 \leq Y \leq 1.75$ & $3.50 \times 3.50$ \\
\hline $\mathrm{SPCl}^{(\mathrm{d})}$ & $Z=195.84$ & $-2.32 \leq X \leq 2.32$ & $-2.35 \leq Y \leq 2.35$ & $4.60 \times 4.60$ \\
\hline SPC2 & $Z=199.84$ & $-2.33 \leq X \leq 2.31$ & $-2.35 \leq Y \leq 2.35$ & $4.60 \times 4.60$ \\
\hline SPC3 & $Z=218.22$ & $-2.28 \leq X \leq 2.35$ & $-2.35 \leq Y \leq 2.35$ & $4.60 \times 4.60$ \\
\hline SPC 4 & $Z=222.22$ & $-2.31 \leq x \leq 2.33$ & $-2.35 \leq Y \leq 2.35$ & $4.60 \times 4.60$ \\
\hline SC3 & $Z=223.70$ & $-2.31 \leq X \leq 2.19$ & $-2.25 \leq Y \leq 2.35$ & $4.50 \times 4.50$ \\
\hline
\end{tabular}

The remaining parts in the slow arm have the $Z$-axis rotated $3^{\circ}$ clockwise. $\mathrm{Z}^{1}$ is the measurement from the center of the slow arm bending magnet to the part. The magnet center has unrotated $\mathrm{Z}=242.20$.

\begin{tabular}{l|ll|l|l|l|l}
\hline SC4 & $Z^{1}=19.20$ & $-2.50 \leq X^{1} \leq 2.50$ & $-2.50 \leq Y^{1} \leq 2.50$ & $5.00 \times 5.00$ \\
SPC5 & $Z^{1}=21.00$ & $-3.50 \leq X^{1} \leq 3.50$ & $-3.83 \leq Y^{1} \leq 3.18$ & $7.00 \times 7.00$ \\
SPC6 & $Z^{1}=25.00$ & $-3.45 \leq X^{1} \leq 3.54$ & $-3.08 \leq Y^{1} \leq 3.92$ & $7.00 \times 7.00$ \\
SPC7 & $Z^{1}=41.19$ & $-3.50 \leq X^{1} \leq 3.48$ & $-3.08 \leq Y^{1} \leq 3.92$ & $7.00 \times 7.00$ \\
SPC8 & $Z^{1}=45.19$ & $-3.50 \leq X^{1} \leq 3.46$ & $-3.85 \leq Y^{1} \leq 3.15$ & $7.00 \times 7.00$ \\
SC5 & $Z^{1}=46.60$ & $-3.25 \leq X^{1} \leq 3.25$ & $-2.25 \leq Y^{1} \leq 2.25$ & $6.50 \times 5.50$ \\
SC6 & $Z^{1}=47.40$ & $-3.25 \leq X^{1} \leq 3.25$ & $-2.25 \leq Y^{1} \leq 2.25$ & $6.50 \times 5.50$ \\
(hodoscope) &
\end{tabular}

(a) All measurements are in inches.

(b) Coordinate system has $\mathrm{Z}$ along radius arm, $\mathrm{Y}$ up, and is right handed. The center is center of target.

(c) C's are scintillators (or hodoscopes). $\mathrm{Z}$ is measured to center and sizes are the active regions. Except for $\mathrm{FCl}$ and $\mathrm{SCl}$, these include a border region of .2 to .43 .

(d) PC's are wire proportional chambers $\mathrm{w} / 2 \mathrm{~mm}$ wire spacings. $\mathrm{Z}$ is measured to proportional plane. Sizes include a border region ranging from .6 to 1.3 wide. All have vertical wires. 
slow arm. Both arms had a momentum resolution of $\pm 5 \%$. The magnet on the fast arm was Argonne's 10 IV 36 septum magnet shimmed to make a 6 " high gap. This ten ton magnet could attain $30 \mathrm{kG}$ at 3000 amps giving $1.120 \mathrm{kG}-i n$. The nine ton slow arm magnet was a $20 \mathrm{~V} 20 \mathrm{C}$-frame magnet. The current limit of 1800 amps allowed a maximum field of $18.5 \mathrm{kG}$ and $439 \mathrm{kG}-\mathrm{in}$. We never ran the magnets more than $96 \%$ of their maximum current capacity.

All counters used were made of Pilot V scintillator connected by UVT lucite light pipes to one of two types of photomultiplier tubes. The eight trigger counters.used 8575 tubes; all others used $6810 \mathrm{~A}$. All bases, except for those used for the time-of-flight, were home-made; the time-offlight (TOF) bases were Ortec 270 constant fraction timing photomultiplier bases. Four bases allowed for three TOF measurements with a resolution of $+.7 \mathrm{~ns}$ (sce Fig. 12 for a typical TOF distribution); the fast TÓF was between FCl and FC5, the slow TOF was between SCl and SC5; and the relative TOF was between FC5 and SC5 (see Fig. 1). The positions and sizes of the trigger counters were determined by a Monte Carlo program. This program predicted that in the worst case $9 \%$ of the elastics that trigger the slow arm will not trigger the fast arm. All counters were powered by three $3 \mathrm{kV}$ power supplies through a system of 50 volt step downs using Zener diodes.

The proportional chambers ${ }^{22}$ had 60 wires for the upstream chambers and 90 wires for the downstream chambers with a wire spacing of $2 \mathrm{~mm}$. Each chamber was run at its optimum voltage in the range of 4-5 kV. 
Magic gas (20\% isobutane, .3\% freon $13 \mathrm{Bl}$, balance argon) with $50 \%$ bubbled through methylal was circulated in series through the chambers. Each chamber wire was connected to a MCl710G differential comparator with a threshold of about $10 \mathrm{mV}$. The negative chamber signal was connected to the inverting input. Then each group of four consecutive wires was connected to a shift register, the integrated circuit MC4012P. The pin configuration for the MC1710G and MC4012P is shown in Fig. 2. When the mode control was set for parallel operation, the information on the wires could be strobed in parallel into the shift registers for an arm if the second and third trigger counters in that arm had a coincidence (later all four trigger counters were used for the fast arm). When a trigger occurred the mode control was set for serial operation to allow the information in all chambers to be clocked out in series into a S.A.C. system. This caused a $1 \mathrm{~ms}$ deadtime before the system could aceept the next trigger and the mode could be reset for parallel operation.

\section{B. Beam and Beam Monitors}

This experiment used a small portion of the primary proton beam split off by two septum magnets. After the splitting, two consecutive bending magnets directed the beam into two quadrapoles for vertical and horizontal focusing. Two final bending magnets bent the beam onto the target. A schematic of the beam line is shown in Fig. 3. All the bending magnets, the septums, and the polarized protom magnet bent the beam to the left 


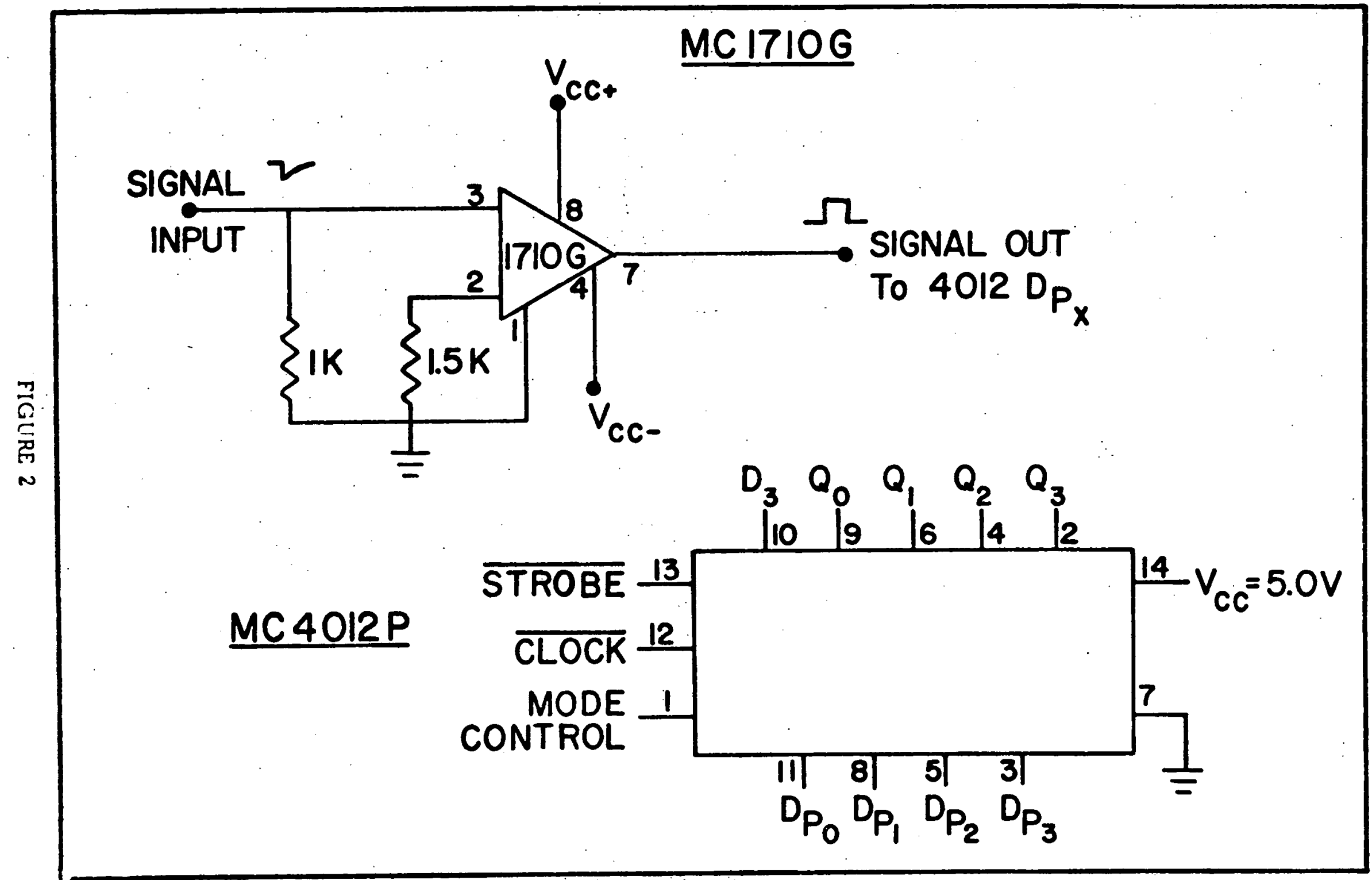




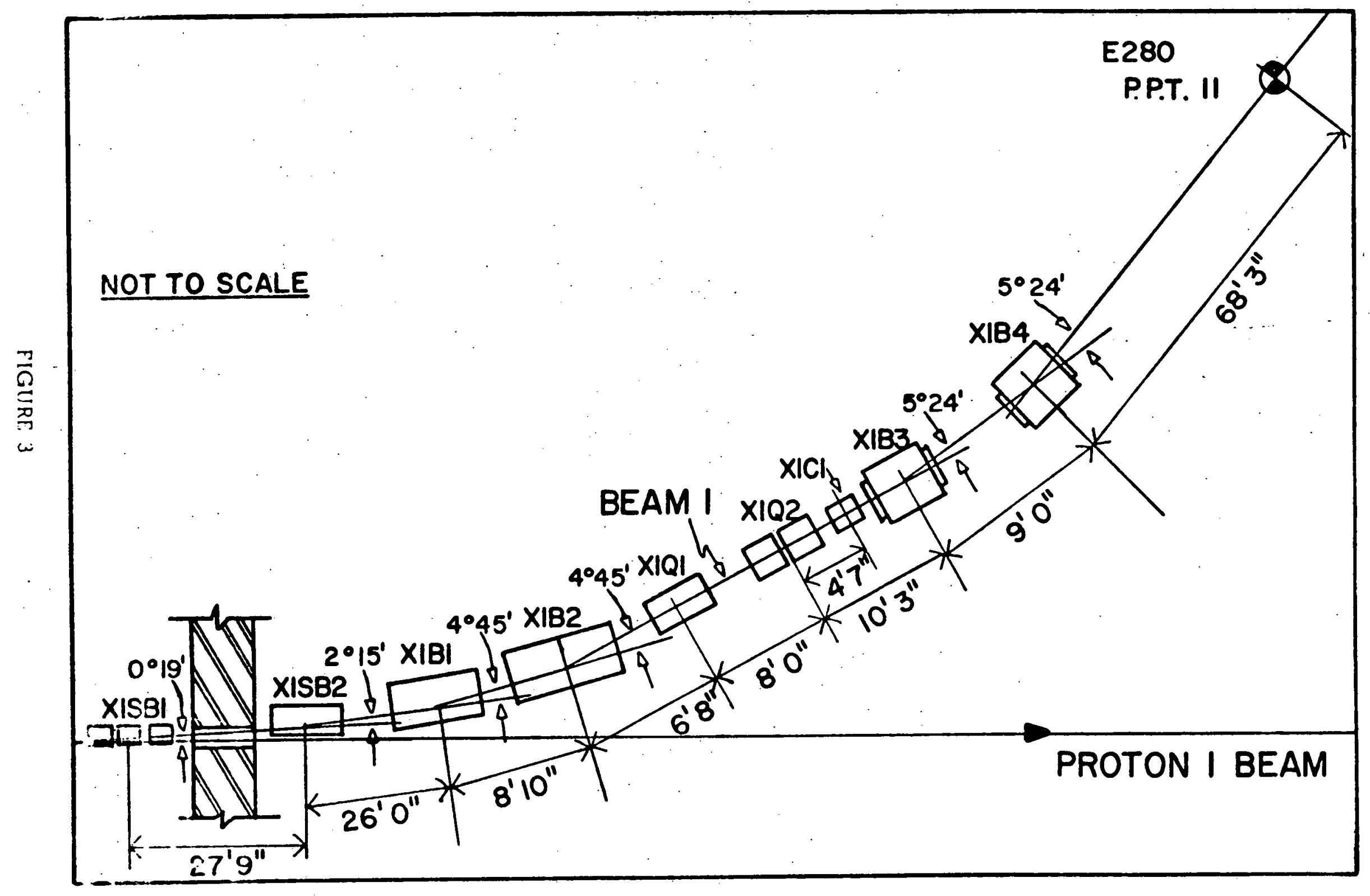


when looking downstream. Twenty feet after the beam hit the target, it plowed into a 12 feet long beam stop that had a five feet deep cavity whose position was adjustable for each incident momentum.

The beam on the target was monitored by up to nine separate devices. The position and size was given by two beam multiwire proportional chambers in which the integrated current on each wire was approximately proportional to the amount of protons in its vicinity. The center and full width at half maximum of both the horizontal and vertical readouts of one of the chambers were available for use.

Two reliable monttors of the beam position and intensity were the individual arm coincidences FA and SA (fast and slow arm). Since less than $.05 \%$ of FA and $1.3 \%$ of SA were from elastic events and the rest of the arm coincidences showed no polarization effects, both $\Gamma A$ and $S A$ (:)uld be used as monitors of beim intensity on the target. l'ig. 5 gives:

a typical sweep of the centroid of the horizontal beam position (HPOS) vs. normalized FA and SA. Comparing this with the plateau in the normalized event-event accidental vs. HPOS of Fig. 4, we can see how FA and SA depend on the beam position at the target.

The primary monitor for beam intensity on the target was BM. Fig. 1 shows the location of BM prior to run 147; afterwards BM was moved to $\sim$ three feet upstream of the target. BM was a $1 / 4$ inch scintillator connected by an air light guide: to a photomultiplier tubs. The signal from the tube was integrated, digitjzed, and recorded on a scaler. The cross sectional area of $B M$ to the beam was the same as the target presented; when 
upstream of the target, $\mathrm{BM}$ could not depend on the polarization of target; when downstream of the target, various tests have shown BM was independent of the target polarization. Before starting up a new momentum, the beam was swept across the target; the number of events (EV), BM, and other values were recorded. Fig. 6 gives such a sweep for BM. Each time BM was moved so that it peaked where EV peaked.

Since the intense beam deteriorated the light emitting properties of the scintillator in BM, BM could only be used as a monitor of beam intensity for runs close in time. Later a water Cerenkov counter, BM', which did not suffer from such deterioration was placed in the original BM position and was positioned like BM.

Two other beam monitors, RM and LM, were three element telescopes. RM was placed at a lab angle larger than the largest lab scattering angle for $\mathrm{p}-\mathrm{p}$ scattering at $5.15 \mathrm{GeV} / \mathrm{c}$ and was aimed at the target. Tests have shown that RM was not sensitive to the polarization of the target. rig.? s hows that RM could also detect the position of the beam. LM looked at a piece of plastic that was positioned so that the piece of the beam that illuminated the target, illuminated the plastic. Initially LM was positioned along the beam line but below it looking up at the plastic. Later it was moved into the shadow of the roof support and looked at the plastic edge on. Since RM was never repositioned or otherwise modified, it could be used as a monitor of the beam intensity (assuming the beam hit the target) for all runs at a given incident momentum even though the runs were distant in time. LM was not only repositioned, but also it was 
EVENT vS BEAM POSITION

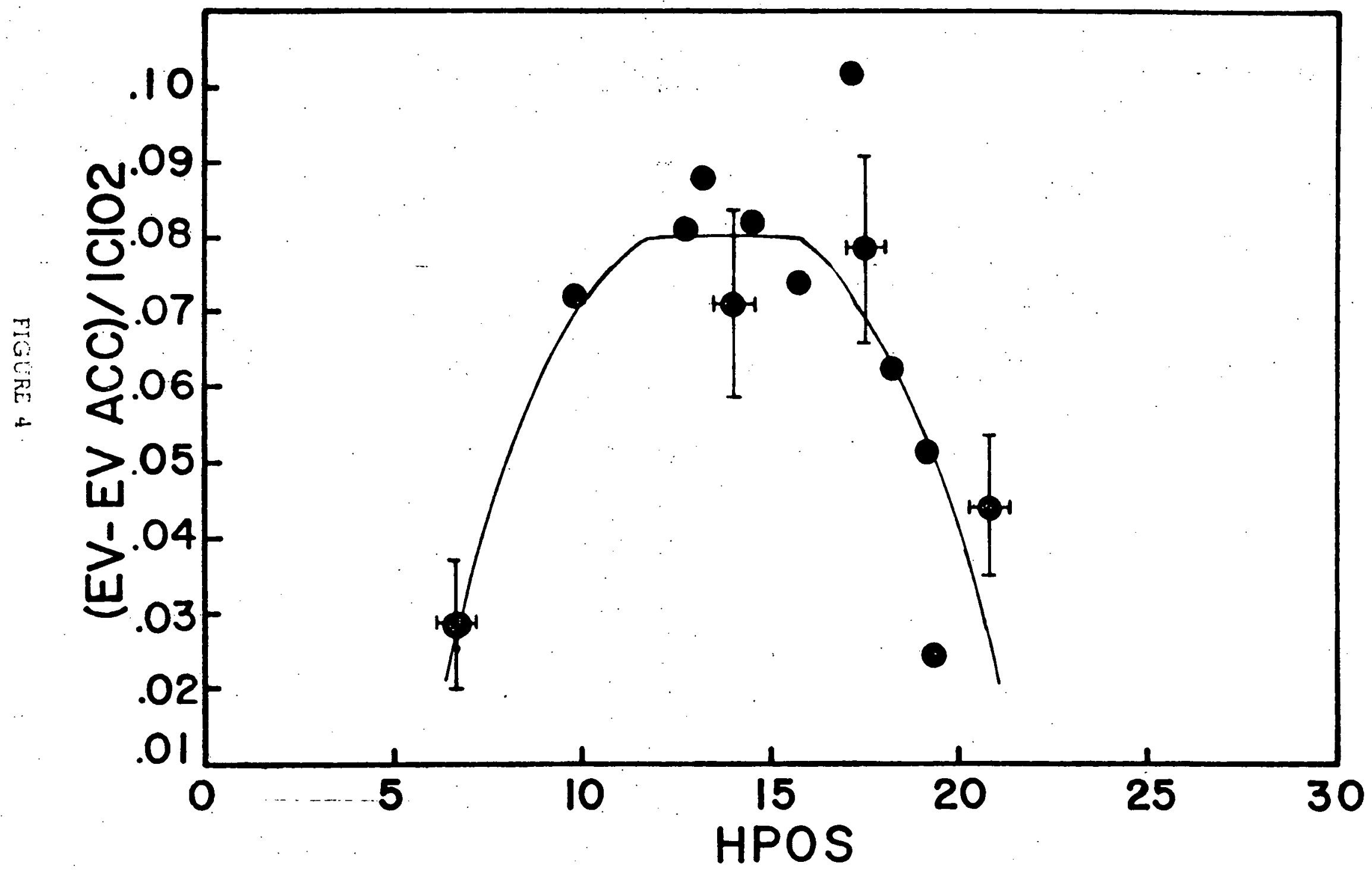


FA \& SA VS BEAM POSITION

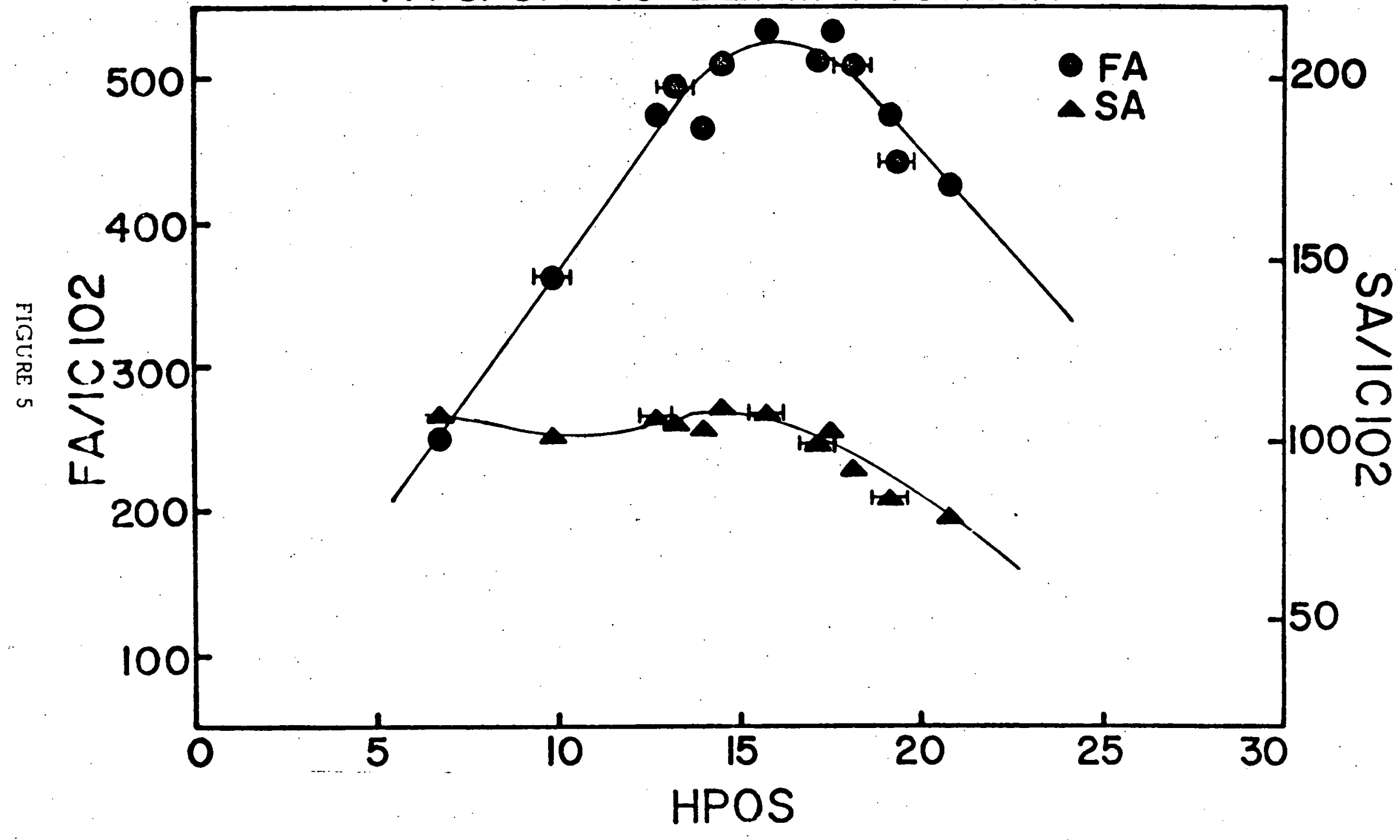




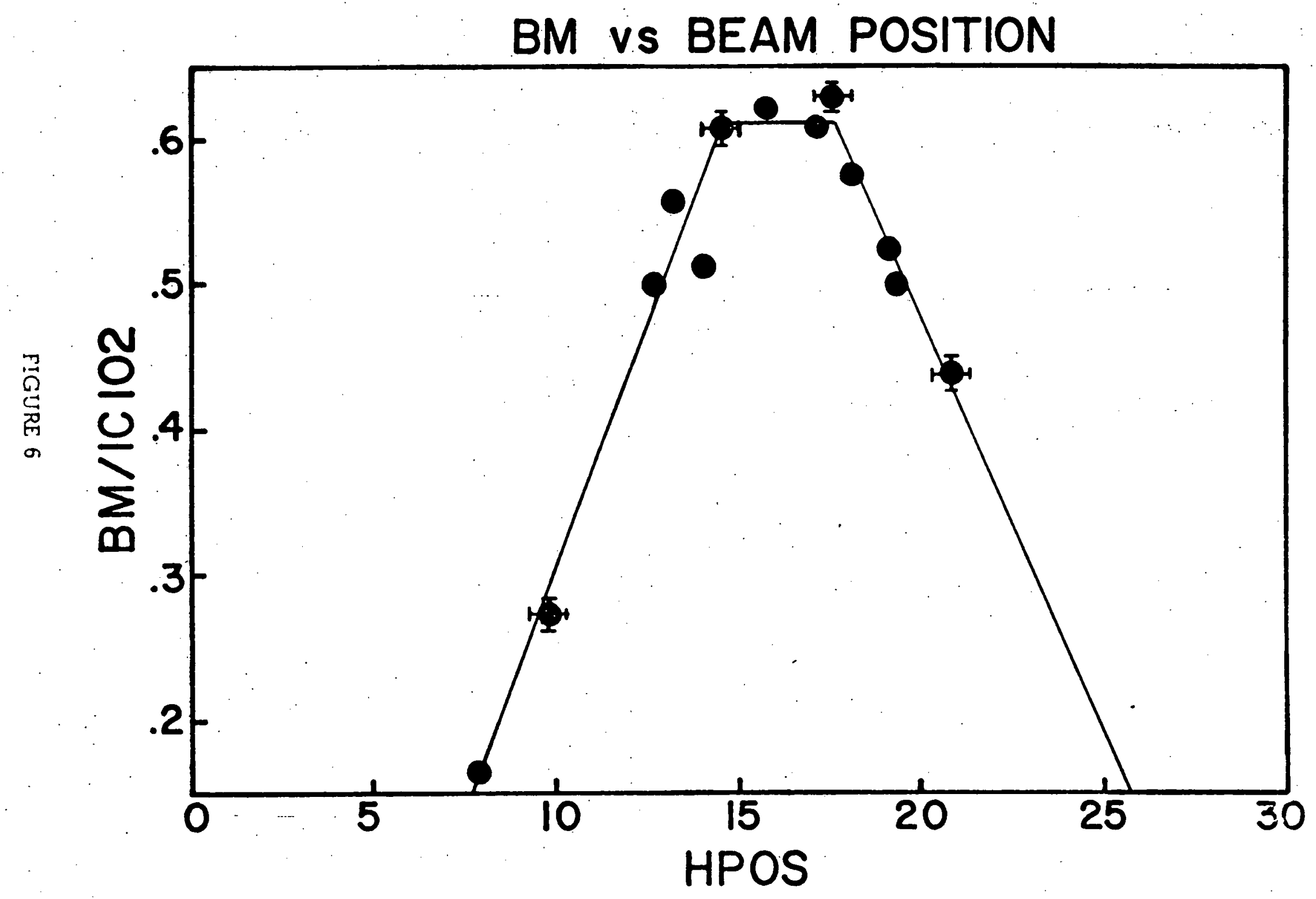




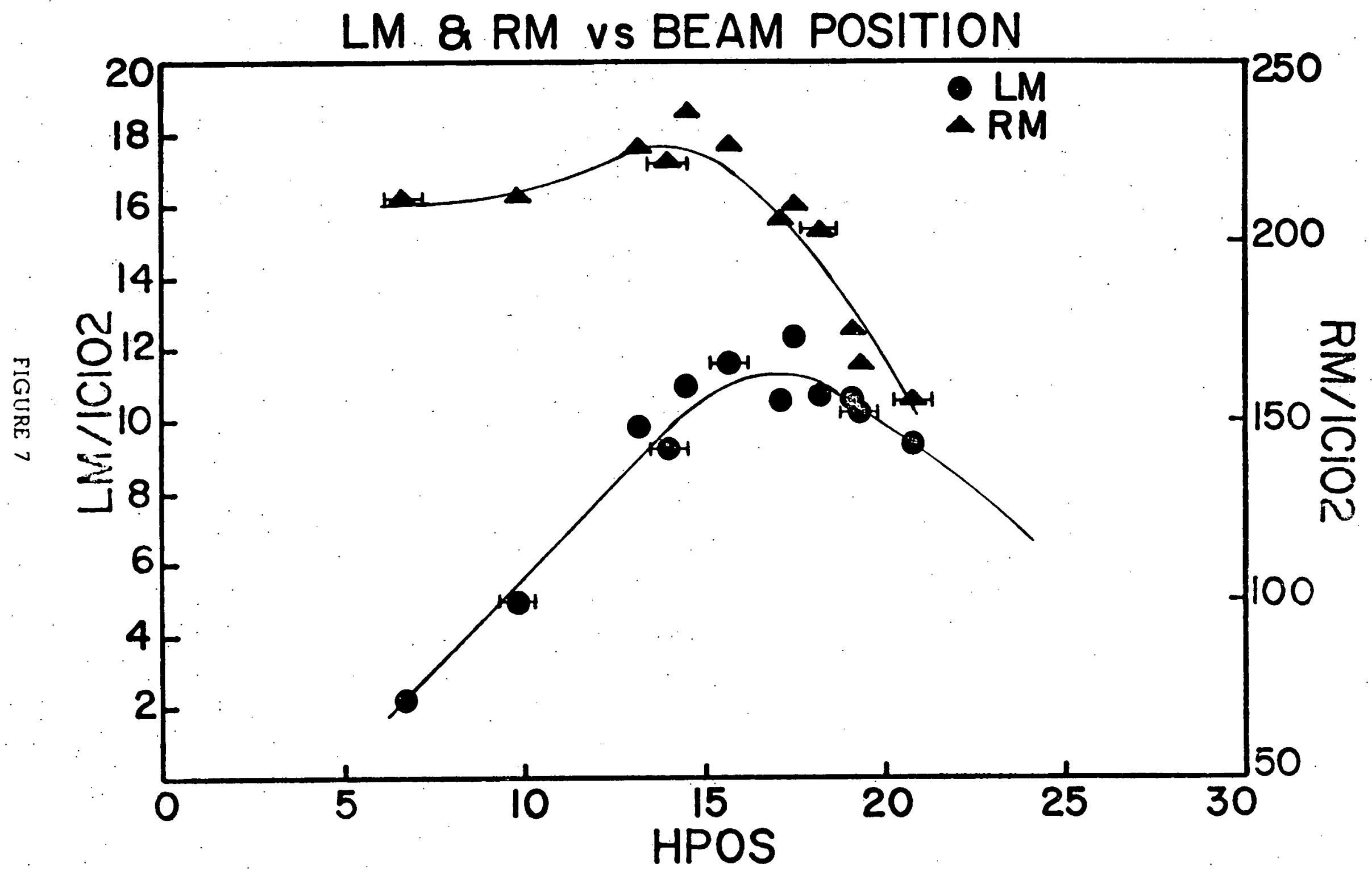


modified to accept a smaller solid angle. So it could only be used as a monitor for runs made under the same conditions.

The beam line used two 1onization chambers, IC102 and IC111, to give a rough estimate of the beam delivered each burst. ICl02, located in the upstream end of the beam line, indicated how much beam was extracted by the septum magnets from the main beam line. The other ion chamber was located about three feet upstream of the PPT magnet yoke. The ion chambers were not the most useful monitors of beam intensity. Several times we calibrated them with gold foil measurements and found that they did not have a linear relationship with the number of protons in the beam. They were useful for sensing change in the amount of beam being extracted in our beam line.

\section{Polarized Proton Target}

This experiment used Argonne's Polarized Proton Target II facility. This consists of a liquid $\mathrm{He}^{4}$ cooled cryostat; temperatures on the order of $1.1^{\circ} \mathrm{K}$ were typical for a target. The target was in a $25 \mathrm{kG}$ field, uniform to less than one gauss over the one inch radius of the pole faces.

The target was 2 in. $\times 1$ in. $x .75 \mathrm{in}$, along the beam, horizontal, and vertical respectively. The composition was $18.4 \mathrm{gr}$. of ethylene glycol, $\mathrm{HOCH}_{2} \mathrm{CH}_{2} \mathrm{OH}, .46 \mathrm{gr}$. of water, and . $38 \mathrm{gr}$. of potassium dichromate, $\mathrm{K}_{2} \mathrm{Cr}_{2} \mathrm{O}_{7}$, a doping material. The target had $9.5 \%$ of free protons by weight.

The potassium dichromate and water formed a free radical, $\mathrm{HCrO}_{4}{ }^{-}$. which was used to help polarize the free protons. The free radicals act 
like free electrons and have such a large magnetic moment that the magnetic field polarizes them nearly $100 \%{ }^{23}$. The spin of the radical and of the free proton can be coupled four ways; the possible energy levels are shown in Fig. 8. The double spin flip transitions are forbidden. Suppose we want to polarize the protons down ( $b$ ). We saturate the system with intense microwave energy of the frequency for $|\downarrow \uparrow\rangle \rightarrow|\uparrow \downarrow\rangle$ transition where in $\mid s_{r} s_{p}>s_{r}$ is the spin of the radical and $s_{p}$ is the spin of the proton. Now the $\mid+t>$ state relaxes through an allowed transition to the $|+\downarrow\rangle$ state.

The method of measuring the polarization has been a subject of dissertations for many years. ${ }^{24-27}$. Here we shall give the method in very general terms. An input signal of definite voltage is sent out to a radio frequency circuit which contains, among other things, a rf oscillator and a resonant clrcuit containing the target. Three output signals are taken. First a background signal, $v_{0}$, near but off the resonance peak is taken by dropping the magnetic field 200 gauss; then a voltage signal, $V_{\text {enh' }}$ ' as the oscillator sweeps through the resonant frequency; finally a $\partial \mathrm{V} / \partial \nu$ signal. The area of the function

$$
f(v)=\frac{v_{\text {enh }}(v)-v_{o}}{v_{\text {enh }}(v)}
$$

is proportional to the polarization. $\mathrm{V}_{\text {enh }}(\nu)$ is very nearly equal to $\mathrm{V}_{0}$ so it is not reliable for this calculation. Instead we reconstruct a $V^{\prime}$ enh $(v)$. $=N \int \frac{\partial v_{\text {enh }}(X, v)}{\partial X} d X$ where $N$ is determined using $v_{\text {enh }}(v)$ as a normalizer and use it instead of $V_{e n h}(\nu)$. The constant of proportionality is 
$\left|S_{r} S_{p}\right\rangle$

$\left|S_{r} S_{p}\right\rangle$

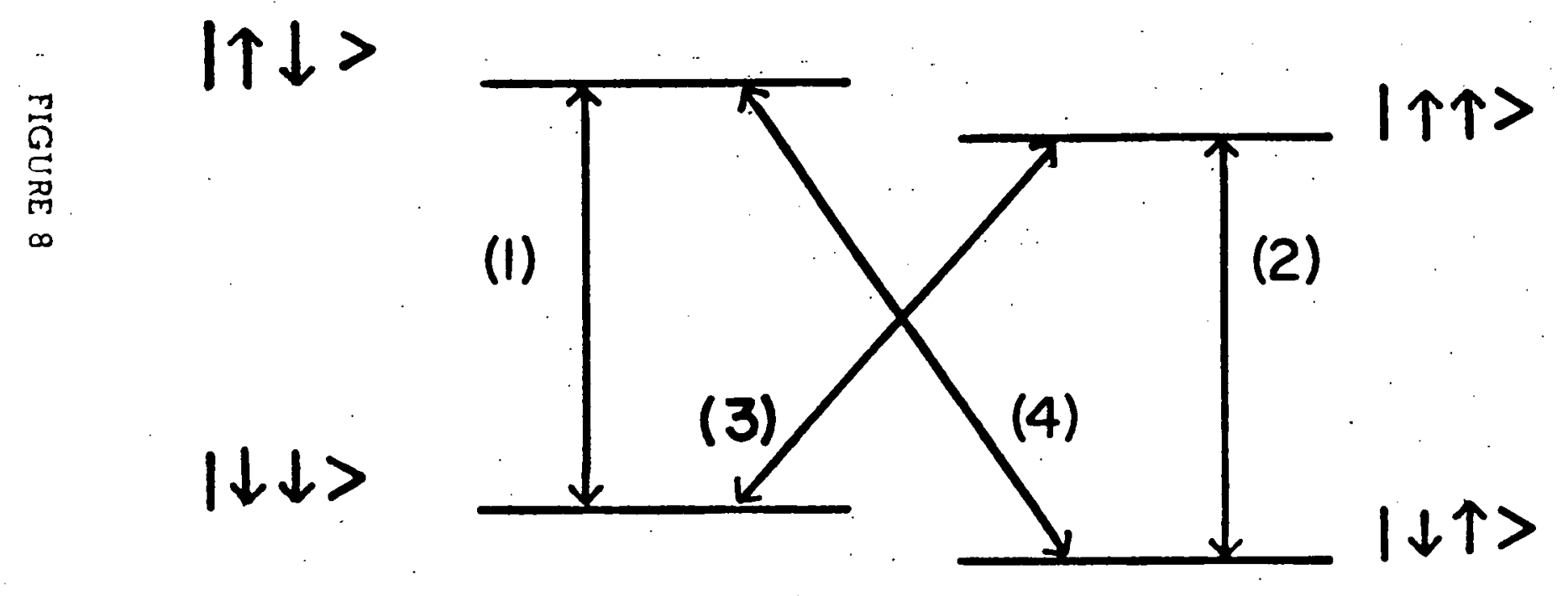


found by measuring the polarization due solely to the separation of energy levels caused by the magnetic field, called the polarization at thermal equilibrium (TE). From statistical mechanics the polarization of spin $1 / 2$ particles in thermal equilibrium can be determined from the relationship

$$
\begin{aligned}
& \quad P=\tanh \frac{\mu \mathrm{H}}{k T} \\
& \mu=\text { magnetic moment } \\
& \mathrm{H}=\text { field strength } \\
& \mathrm{k}=\text { Boltzmann constant } \\
& \mathrm{T}=\text { temperature } \\
& { }^{\circ} \mathrm{K}
\end{aligned}
$$

The theoretical and measured values of the thermal equilibrium polarization then determines the constant of proportionality.

The polarization of each target decayed exponentially with accumulated flux $\varphi$, according to the relationship

$$
P=P_{0} e^{-\varphi / \varphi_{0}} \quad \varphi_{0}=\text { characteristic flux }
$$

Using four targets for which we calibrated the beam flux using gold foil measurements, we found that $\varphi_{0}$ did not appear to be dependent on the incident energy.

\section{Electronics}

The fast logic used in the experiment is shown in Fig. 9. A typical non-TOF counter had its $5 \mathrm{~ns}$ clipped output sent to a discriminator with about a $100 \mathrm{mV}$ threshold and a $5 \mathrm{~ns}$ width cable; one output went to a coincidence on the floor of the experiment, the other to the electronics trailer. 


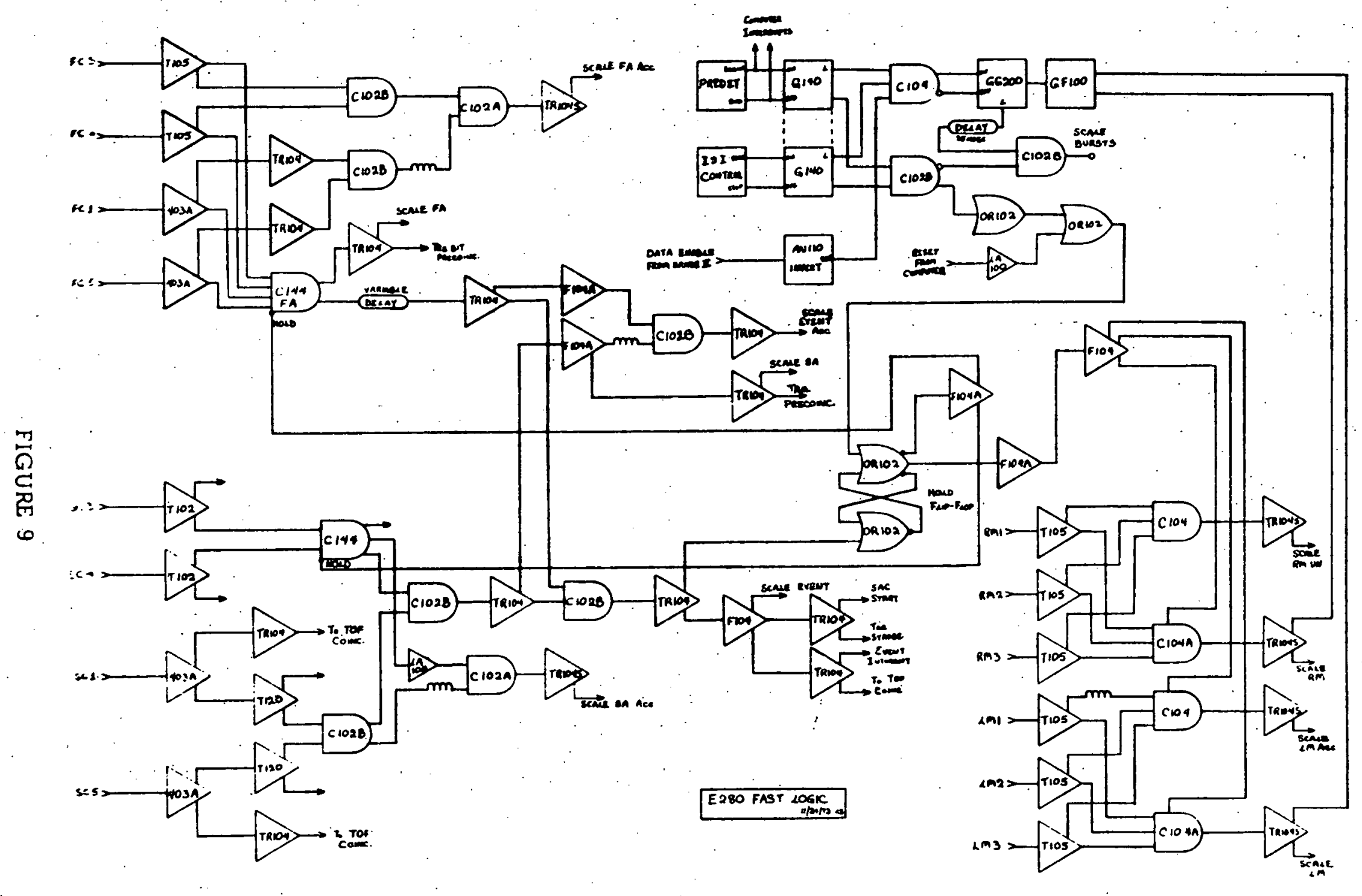


Each arm had a four-fold coincidence, FA for the fast arm, SA for the slow arm. FA and SA were formed very close to the proportional chambers to help reduce the dead time of the system. On the slow arm SCl and SC5 were TOF counters which formed the dual coincidence $\mathrm{SC} 1 \cdot \mathrm{SC} 5 ; \mathrm{SC} 3 \cdot \mathrm{SC} 4$ strobed the slow arm chambers and in coincidence with $\mathrm{SCl} \cdot \mathrm{SC} 5$ formed SA. A SA accidental $\left(\mathrm{SA}^{*}\right)$ was formed by delaying $\mathrm{SCl} \cdot \mathrm{SC} 5$ with respect to $\mathrm{SC} 3 \cdot \mathrm{SC} 4$ by a rf cycle of the ZGS.

On the other hand, FA was a direct four-fold coincidence composed of FC1 - FC3 - FC4 - FC5. This signal was used to strobe the fast arm chambers and to form the event and event accidental. FA accidental (FA*) was made independently of FA by forming a FCl $-F C 5$ and delaying it with respect to $\mathrm{FC} 3 \cdot \mathrm{FC} 4$.

The event coincidence (trigger) was formed on the floor of the experiment by putting SA in coincidence with a delayed FA. The delay in FA was variable and remotely controlled, so we could calibrate this delay by a timing curve. The event accidental (EV*) was formed by further delaying $8 \mathrm{~A}$.

LM and RM were two simple three-fold gated coincidences of discriminated counter output. $\mathrm{LM}^{*}$ was the accidental for LM. RM UN was an ungated three-fold RM coincldence. The hold gate was generated by a flip-flop using as inputs a reset pulse and the event coincidence. The reset was generated by the computer, or by a beginning of burst, or by turning on the scalers. Other quantities such as FA, FA*, SA, SA*, EV, and $\mathrm{EV}^{\star}$ were also gated off using the hold gate. 
The signals for the time-of-flight system and the hodoscope tagging units were derived from discriminated counter pulses that were strobed by FA for the fast arm counters and SA for the slow arm counters. The start and stop pulses for the TOF were further strobed by EV. Each of the three TOF systems used a time-to-pulse-height converter and an analog-todigital converter.

\section{E. Data Acquisition and Monitoring}

The most important apparatus for data monitoring and data acquisition was our PDP-15 computer. It collected the data, built 46 event buffers, and then recorded the buffers on magnetic tape. For each event information regarding the chamber track locations, the hodoscope signatures, time-of-flight, transducer voltages, and scaler readings were recorded. For every buffer a NMR sweep of the target polarization was made and recorded. On-line displays were avallable for the chamber hit distributions, hodoscope frequency distributions, NMR sweeps, time-of-flight distributions, the buffer built for each event, and numerous transducer voltages. In addition to the on-line target polarization measurements, extensive measurements of the target polarization were made at regular intervals utilizing a sophisticated off-line program.

When a trigger occurred these things happened. First the trigger gated off the experiment and sent an interrupt to the computer which was immediately serviced. Also it started the clocking of the data in the chamber shift registers into the SAC system. After the information was in 
the SAC system, the computer began reading out the information on the data chain. These data included the fixed data, various voltages, accumulator scalers, SAC scalers, and TOF scalers. Upon finishing the scan of the data chain, the computer sent out a signal to restart the experiment. In addition to accumulating data through the computer various quantities were scaled and recorded by hand. These included FA, FA*, SA, SA*, EV, $E V^{\star}, L M, L M^{*}, R M, R M U N, 1 C 102, I C 111, B M, B M^{\prime}, H P O S$, and BURST.

F. Problems and Execution

All the major problems encountered during the experiment were beam related. The first problem noticed was a general radiation background not associated with the target and which apparently came from both upstream and downstream of the target. Initially the beam diagnostics were deficient. With the use of polaroids we discovered that in several places the beam even when hitting the target was also hitting the beam vacuum pipes. The problem was ameliorated by installing more complete beam diagnostics in order to steer the beam better and by installing a horizontal and vertical collimator in the second septum magnet to reduce the size of the beam entering the vacuum pipe. The downstream problem was caused by an inappropriately designed beam stop. To help, the stop was redesigned with a five feet deep hole to let the unscattered beam through. So any backscattered beam had to go through five or more feet of lead to get back to the detectors. These improvements were shown to have helped reduce the background by a factor of $\sim 100$. 
During the course of the experiment other improvements were made in the experimental equipment and setup. A stiffener power source was added to the last four stages of the counter bases; this allowed the typical detection rates in the counters to go from 1-5 megacycles to 6-10 megacycles. To decrease the number of tracks in the chambers and to help cut down on accidental triggers we made copious use of lead. On the back and side of the fast arm nearest to the beam stop, 8-12 inches of lead was stacked to shield against back scattering from the beam stop. Also a collimator directly upstream of $\mathrm{FCl}^{\prime}$ was used to decrease rates in $\mathrm{FCl}$ by a factor of $2 / 3$. To further get rid of the lower momenta pions and protons which were shown to be the biggest source of background from the target, a small magnet was placed between the target and the collimator on the fast arm; this magnet bent the elastic particles $1 / 2^{\circ}$ to $1^{\circ}$ in the same direction as the spectrometer magnet and forced most of the lower momenta particles outside the acceptance of the system. A final improvement in the fast arm consisted in replacing the two three-element hodoscopes with three 15element hodoscopes. These aided in the selection of the correct tracks during the reconstruction.

Typically the running procedure was as follows. First the spectro-

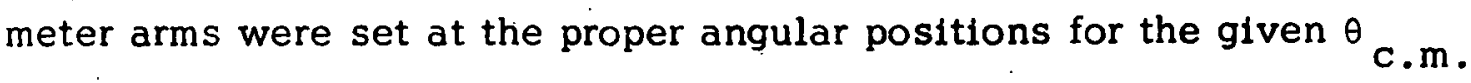
scattering angle; also the two spectrometer magnets were set at the currents that bent the kinematically proper momentum particle $3^{\circ}$ (or at the maximum current of the magnet) and the remote delay for FA was set. (During the times we used the sweeping magnet on the fast arm we also 
properly positioned the sweep magnet and its current.) All these values were predetermined by a kinematic and interpolation program SUM280. The angular positions and remote delay were also checked several times by doing sweeps in these variables. In all cases in which the energy loss of the scattered (recoil) protons in the target was insignificant, the angular positions were shown to be properly determined. We then ran an even number of files having opposite enhancements of the target. Each file for an angle would have about the same number of triggers. Normally the number of triggers was from 1500-3000; the number of triggers (in the above range) was chosen to give about 30 minute runs. 


\section{References}

22. R. R. Crittenden and J. C. Krider, Indiana University Proportional Chambers, Technical Report C00-2009-49 (unpublished).

23. M. Borghini, Polarized Targets and Ion Sources (edited by La Direction de la Physique), p. 133.

24. C. H. Schultz, Scattering of $250 \mathrm{MeV}$ Positive Pi Mesons from a Polarized Proton Target (Ph.D. thesis), UCRL-11149.

25. H. E. Dost, Measurement of the Spin-correlation Parameter $C_{N N}$ in Proton-proton Scattering at $680 \mathrm{MeV}$ (Ph.D. thesis) UCRL-11877?

26. T. M. Powell, Search for Polarization in Elastic e $\mathrm{e}^{-} \mathrm{p}$ Scattering at 15 and $18 \mathrm{GeV}$ (Ph.D. thesis), UCRL-20093.

27. C. C. Morehouse, Photoproduction of $\pi^{+}$Mesons from a Polarization Proton Target at 5 and $16 \mathrm{GeV}$ (Ph.D. thesis), UCRL-19897. 


\section{Analysis}

The analysis of the experiment was done primarily on Indiana University's CDC-6600 computer with several runs analyzed on an offline PDP-15 computer. The two main programs used were E280, a reconstruction program, and DST280, a program used to select the elastic events. Changes in beam position, variations in beam intensity, and other such experimental variations necessitated some data selection. This data selection depended on the scalers recorded for each run.

\section{A. Scaler Data}

All the scaler readings (including the fast arm coincidences, slow arm coincidences, BM, LM, RM, BMP, events, and the summed horizontal beam position) were transferred to IBM cards to facilitate their use. First the cards for each run were processed through the program SCALR. SCALR printed for every run all the scaler readings and the ratios of each combination of scalers (with the accidentals being used only as a subtraction term). Also it printed columns of selected ratios for all runs at the end. This enabled us to detect most of the mispunched data and some of the misrecorded data.

Then for all the runs at a given angle the program BADDIES was used. BADDIES used the scaler cards and the card output from the analysis program to detect bad runs. For the important ratios, scalers, or other numbers, such as the number of elastics for a run, the program indicated for which runs this varlable was $2,5,10,20$, or $30 \%$ away from the average value for that angle. Thus an unusual variable was instantly noticeable. In addition, this program checked for systematic errors. No beam 
monitor should depend on the polarization. If we let $E=+1$ for positive enhancement of the target and $=-1$ for negative enhancement. The sum

$$
\sigma=\sum_{i} E_{i} f_{i} / \sum_{i} f_{i}
$$

summed over an equal number of positive and negative enhancements where $f_{i}=F A / B M, S A / B M$, or $B M P / B M$, all beam monitors, should be 0 . Typically $|\sigma|<.01$ where the larger values occurred for the angles with few runs.

$\mathrm{FAC} / \mathrm{SAC}\left(\mathrm{FAC} / \mathrm{SAC}=\left(\mathrm{FA}-\mathrm{FA} A^{*}\right) /\left(\mathrm{SA}-\mathrm{SA}^{\star}\right)\right)$. was the single most important ratio because it was sensitive to any change in the arm coincidences, movement of the beam (see Fig. 5), and very high beam intensities (FA would saturate). For an angle we deleted any run which had a FAC/SAC greatly different from the average. The selection was not according to a rigorous criterion and was somewhat subjective. Typically all the accepted runs had FAC/SAC values grouped together; any unacceptable run would have a value noticeable distant from the group. The other ratios considered important were $F A C / B M, S A C / B M, L M / B M, R M / B M$, and BMP/BM. Deviations from an acceptable FAC/SAC value were always accompanied by deviations from acceptable values in some or all of the other important ratios.

\section{B. Analysis Program}

The analysis of the data was done using two programs, E280 and DST 280. E280 was a reconstruction program. Inttially E280 read the data tape, unpacked the information, and stored the data in an array. Then 
the information for an event was transferred to a routine which reconstructed the information from the proportional chambers into points in space called sparks. A filtering routine, discussed later, looked for a track in each arm. A successfully reconstructed and filtered event was one with a track in each arm.

Next various kinematic properties of the event were calculated. From the angle of bend through each spectrometer magnet, the momenta of the two final state protons could be calculated. The three TOF's were calculated from the raw TOF data. The scattering angles were calculated. Finally for every reconstructed event a data summary record was written on tape. It contained $(\Delta \mathrm{P} / \mathrm{P})_{S}=\left(\mathrm{P}_{\mathrm{S}}-\mathrm{P}_{O S}\right) / \mathrm{P}_{\text {os }}$, where $\mathrm{P}_{\mathrm{S}}=$ measured slow proton momentum and $P_{\text {os }}=$ predicted slow momentum, $(\Delta P / P)_{f}=\left(P_{f}-P_{o f}\right)$ $/ P_{\text {of }}$, where $\mathrm{P}_{\mathrm{f}}$ and $\mathrm{P}_{\text {of }}$ are the corresponding fast momenta, the three TOF's, $\Delta \theta_{\mathbf{S}}$ (the measured slow scattering angle - the angle predicted using the measured fast angle), mass correlations for the two protons, and four points along with four vectors that completely determine the tracks.

After we had reconstructed each spark in every chamber using standard techniques, we had to filter out the tracks from the ensemble of sparks available. First the flltering routine calculated the effective center for each arm. The effective center for an arm was calculated by projecting the track of an "Ideal" particle outside the polarized proton magnet backward through the magnet to the perpendicular to the arm from the center of the target. An "ideal" particle is the result of an elastic 
collision at the given $\theta_{\mathrm{c} . \mathrm{m}}$. at the center of the target.

Now the line between the effective center of an arm and the first spark in the first chamber was projected to the fourth chamber. If no spark could be found in that chamber within .2" of the intersection, the projection was done to the third chamber with the same criterion. If no such spark could be found in the third chamber, subsequent sparks in the first chamber were used, then the second chamber's sparks if necessary. The established track into the spectrometer magnet was then projected to the center line of the magnet. The intersection point was used just like the effective target center in conjunction with the last four chambers to find a track after the magnet. When a track was found in both arms, we had a filtered event. In the case no track could be found, the event could not be successfully filtered.

\section{Determination of the Polarization}

The analysis program, E280, did not calculate the polarization, but it did write summary tapes. These summary tapes were processed by the program DST280. DST280 allowed for the selection of elastic events using cuts on six variables, $(\Delta P / P)_{f},(\Delta P / P)_{s}, \Delta \theta_{s^{\prime}}$, relative TOF, fast TOF, and slow TOF. Any number of combination of cuts (within the capacity of the computer) were allowed, however we chose four: The cuts were chosen to include as many elastics as possible, but not at the expense of including significant background. Histograms of the six variables were printed for every cut for every run. Examples of the histograms for the $(\Delta P / P)_{f},(\Delta P / P)_{S}$, relative $T O F$, and $\Delta \theta_{S}$ before and after cuts are given 
for a $7.0 \mathrm{GeV} / \mathrm{c}$ run in Fig. 10-13. The program punched a card for every cut giving the run number, momentum, angle, and number of events passing the cut.

The scaler cards and punched cards from DST280 and E280 were now ready for the calculation of the polarization. Now the program used was DATSUM. DATSUM had four major objectives. First, the most important scalers and scaler ratios were again printed. Second, for each angle the runs of opposite enhancement were paired and the polarization was calculated for each pair according to the formula

$$
P=\frac{1}{P_{T}} \frac{E_{u}-E_{d}}{E_{u}+E_{d}}
$$

where

$$
\begin{array}{ll}
E=\frac{\text { number events passing cuts }}{\text { monitor counts }} \times \frac{\text { number of triggers }}{\text { number of triggers analyzed }} \\
u=\text { spin up } \\
\mathrm{d}=\text { spin down }
\end{array}
$$

$B M, F A$, and SA were used as the beam monitors. The polarization was also calculated using $E^{1}=E A / R$ where

$$
\begin{aligned}
& A=\text { number of events analyzed } \\
& R=\text { number of events reconstructed }
\end{aligned}
$$

R/A was a measure of the chamber efficiency and so served as a correction factor for the chambers.

The final data selection was done in DATSUM. Our objective now was consistency in the scaler ratios and other important numbers such as $R / A$. Specifically we wished that both runs in a pair have similar ratios. 


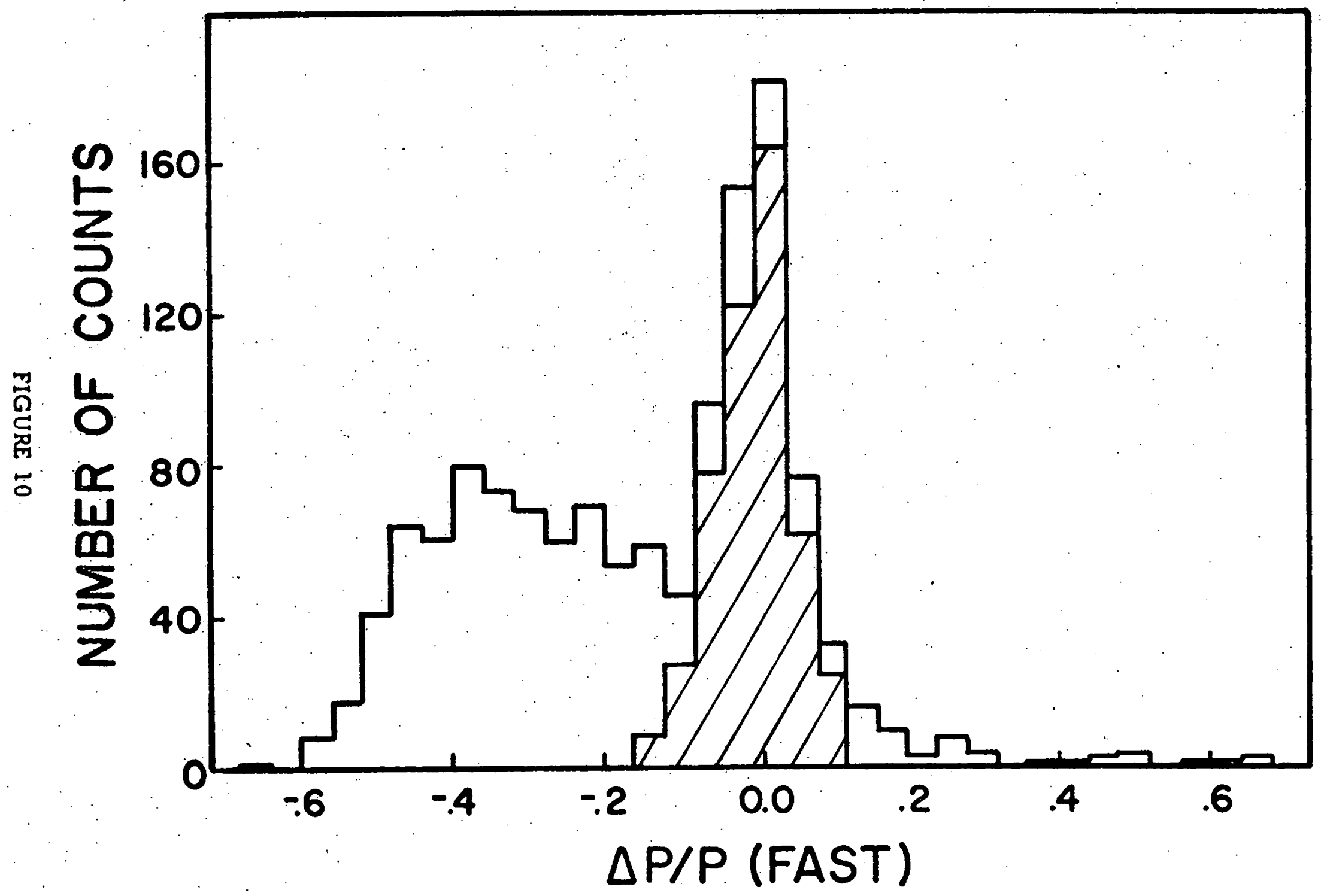




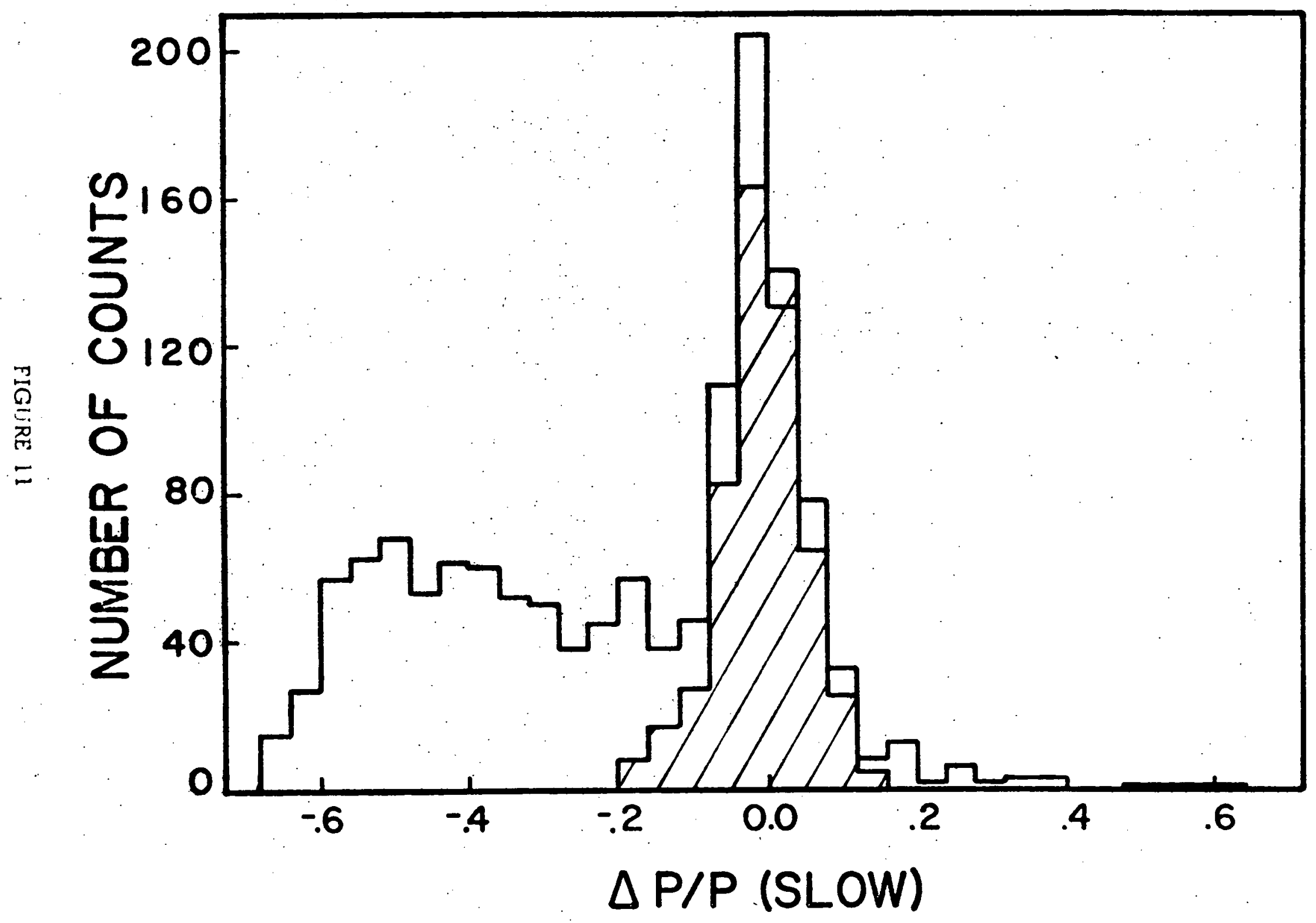




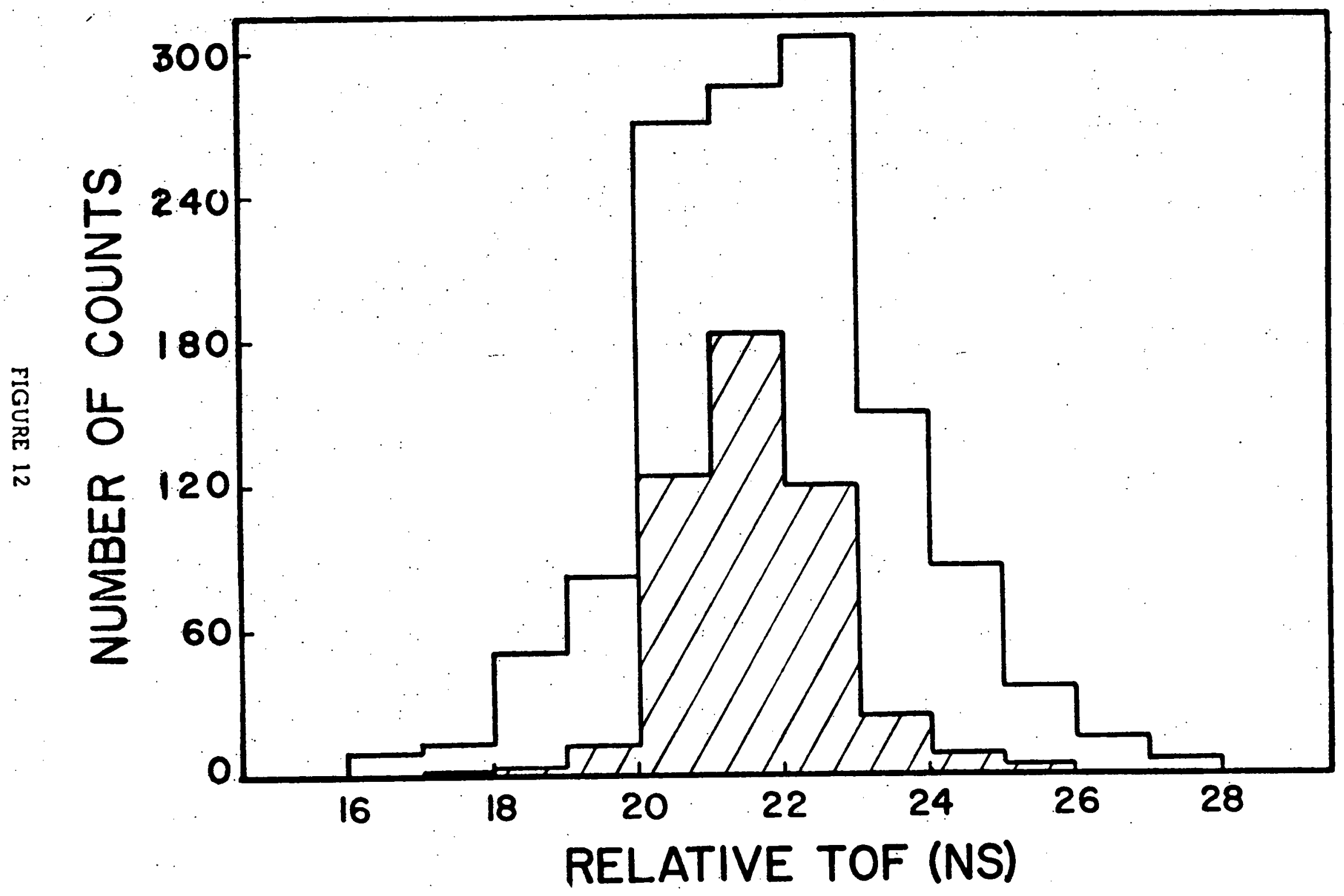




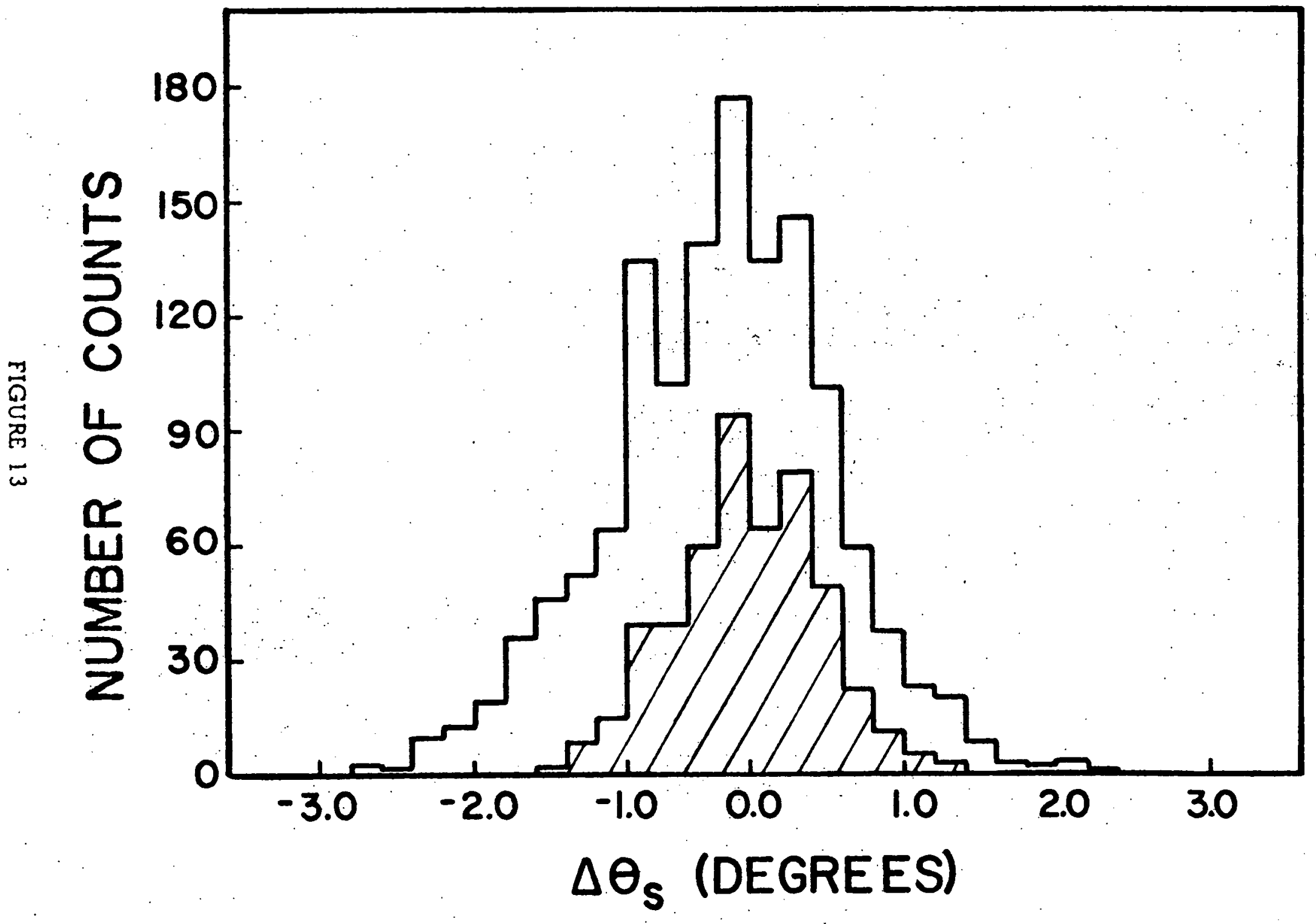


Larger differences were tolerated between pairs. The reason for this was that whenever a change did occur we could be sure there was an equal number of runs of an enhancement before the change and an equal number of runs of an enhancement after the change. For an example suppose on run 6 of an angle the beam intensity increased. Thus for runs 1-5 we had an $R / A \approx 75 \%$ and for run 6 and after $R / A \approx 50 \%$. Then the match pair 5 and 6 would have very different $R / A$ and so deleting run 5 would match the $R / A$ values better.

To calculate the polarization for a run an exact relationship was used. $^{26}$ The starting point is the equation

$$
N_{1}=M_{1} I_{0}\left(1+P_{i} P\right)+B_{1}
$$

where

$$
\begin{aligned}
& N_{1}=\text { number of events passing cuts for the } i^{\text {th }} \text { run } \\
& M_{1}=\text { number of monitor counts } \\
& I_{0}=\text { number of elastics per unit monitor count for an unpolarized } \\
& \quad \text { target with the particular geometry used } \\
& P_{i}=\text { polarization of the target } \\
& P=\text { polarization of } 100 \% \text { polarized target (i.e. the answer) } \\
& B_{i}=\text { number of background counts }
\end{aligned}
$$

The number of elastics per unit monitor is given by

$$
E_{i}=\frac{N_{i}-B_{1}}{M_{i}}
$$

We would like to find a suitable $I_{0}$ and $P$ such that for all the runs for the angle 


$$
E_{i} \approx I_{0}\left(1+P_{i} P\right)
$$

Therefore we should minimize the sum

$$
T=\sum_{i} M_{i}\left[E_{i}-I_{0}\left(1+P_{i} P\right)\right]^{2}
$$

with respect to $I_{0}$ and $P_{\text {, }}$ where the runs are weighted by the monitor counts so the longer runs in amount of beam are more important.

$\mathrm{T}$ will be minimized when

$$
\partial \mathrm{T} / \partial \mathrm{I}_{0}=0
$$

and

$$
\partial \mathrm{T} / \partial \mathrm{P}=0
$$

Eq. 22 gives the equation

$$
\frac{\sum_{i}\left(N_{1}-B_{1}\right)}{\sum_{i} M_{i}}+\frac{P \sum_{i}\left(N_{1}-B_{1}\right) P_{1}}{\sum_{i} M_{i}}=I_{0}+2 P P_{T}+P^{2} P_{T}^{2}
$$

The next equation gives

$$
\frac{\sum\left(N_{i}-B_{i}\right) P_{i}}{\sum_{i} M_{i}}=I_{O} \overline{P_{T}}+I_{0} P \overline{P_{T}^{2}}
$$

where

$$
\overline{P_{T}^{n}}=\frac{\sum_{i} M_{i} P_{i}^{n}}{\sum_{i} M_{i}}
$$

These two equations can be solved for $\mathrm{P}$ to give

$$
P=\frac{A}{1-A \widetilde{P_{T}}}
$$

where

$$
A=\frac{\sum_{i}\left(N_{i}-B_{i}\right) \Delta_{i}}{\Delta^{2} \sum_{i}\left(N_{i}-B_{i}\right)}
$$




$$
\begin{aligned}
& \Delta_{i}=P_{i}-\overline{P_{T}} \\
& \Delta_{2}=\overline{P_{T}^{2}}-\bar{P}_{T}^{2}
\end{aligned}
$$

This equation reduces to the form used in the pairwise matched runs of the other part of DATSUM if we assume all $M_{i}$ are constant and $\left|P_{i}\right|$ is a constant $=P_{K}$ then $\Delta_{i}=P_{i}, \Sigma \underset{i}{\Delta} B_{i}=0$ (i.e. the background is unpolarized), $\overline{\mathrm{P}_{\mathrm{T}}}=0$, and $\overline{\mathrm{P}_{\mathrm{T}}^{2}}=\mathrm{P}_{\mathrm{K}}^{2}$ so

$$
P=A=\frac{\Sigma N_{i} P_{i}}{P_{K}^{L} \Sigma N_{i}}=\frac{1}{P_{k}} \frac{\Sigma N_{d}-\Sigma N_{u}}{\Gamma N_{d}+\Sigma N_{u}}
$$

Finally DATSUM gave us an estimate of the asymmetries of the system. This was done by using all the positive enhancement runs and equation 13 to calculate the polarization. For this calculation we artificially took every other positive enhancement as a negative enhancement. The result should be zero. The same calculation was done using only the negative enhancement runs.

\section{Background Studies}

The background was studied by three methods. The first method used a dummy target. At each momentum we ran a target empty run and then several carbon target runs at selected angles. Since the carbon target was not equivalent to a normal target without its free protons (it was the same size but not massive enough), we had to subtract from the carbon run the empty target run to find what background was due to the carbon, correct for the mass difference, then add in the empty target run. Note that the same cuts in DS'1280 used for an angle were used in the empty 

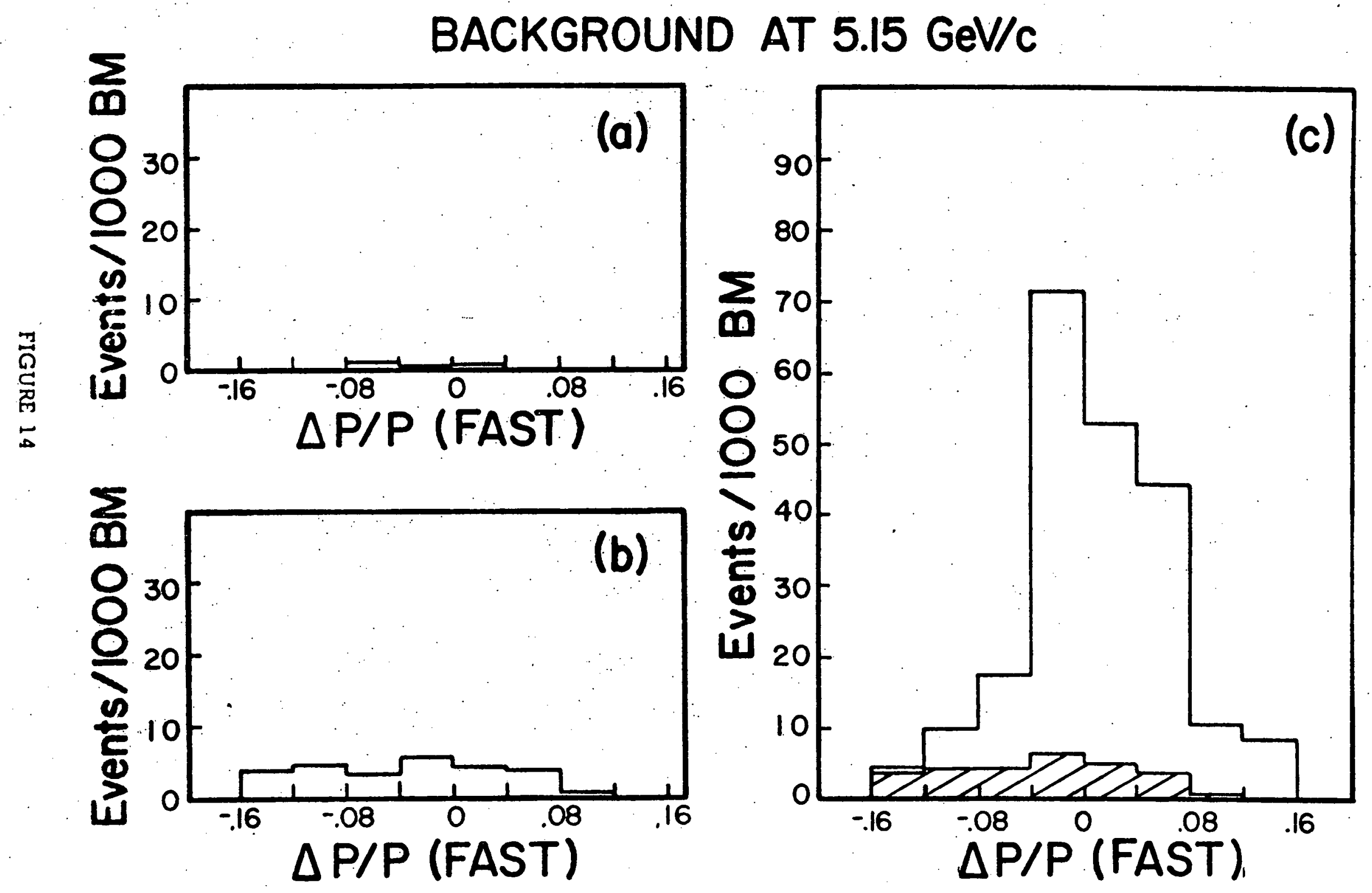


\section{BACKGROUND AT $7.00 \mathrm{GeV} / \mathrm{c}$}

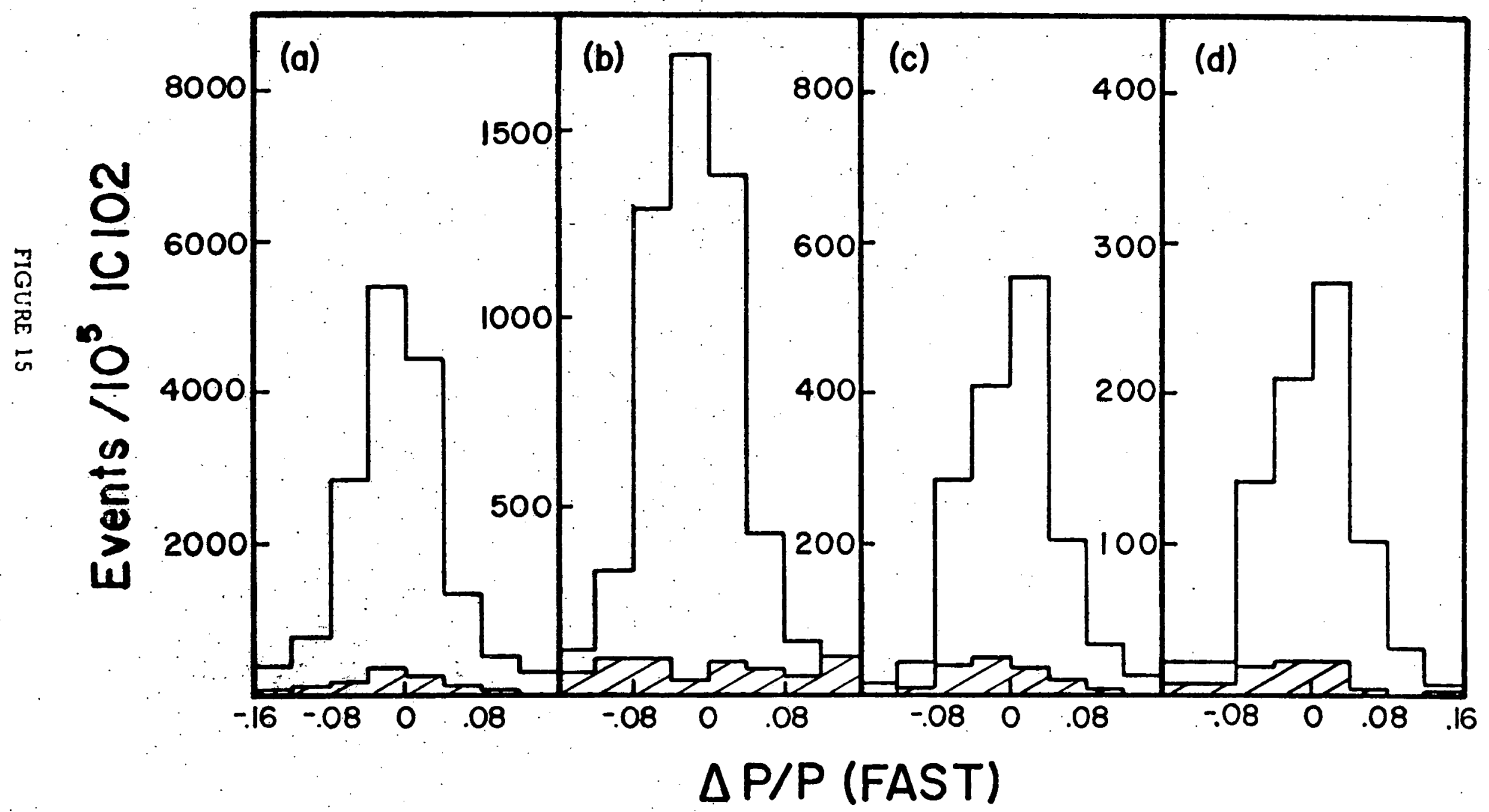




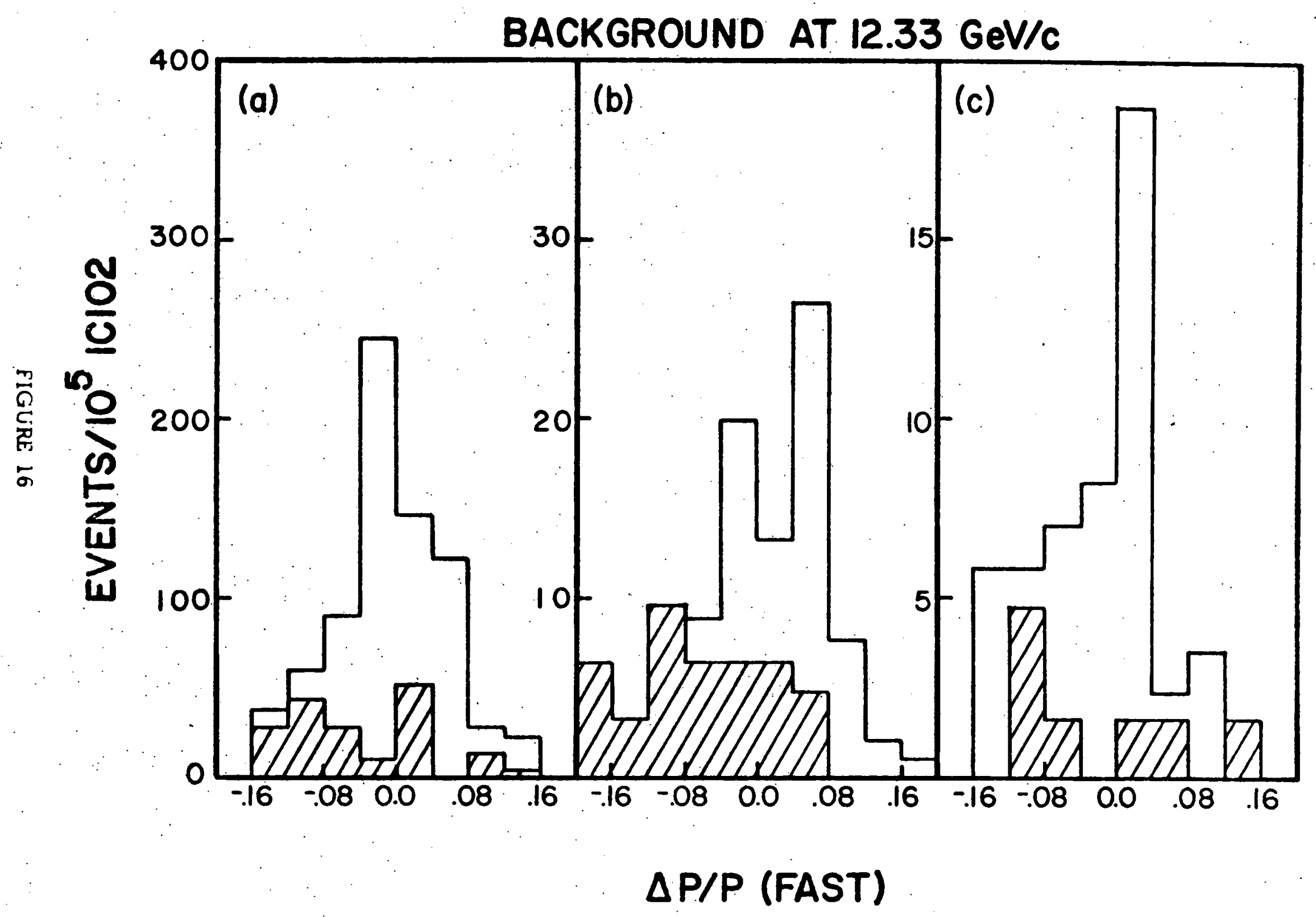


and carbon targets to determine the background so the number derived could be directly applied to the results of runs at the angle.

The second method was a Monte Carlo calculation of the background. The program assumed a momentum dispersion of $\pm .005 \mathrm{GeV} / \mathrm{c}$ for the incident beam. Events of the reaction $p p \rightarrow p p$ were generated with free protons and bound protons with a mean Fermi momentum of $216 \mathrm{MeV} / \mathrm{C}$ in the proportion seen by the beam. For all events that could trigger the system, histograms of $(\Delta P / P)_{f^{\prime}}(\Delta P / P)_{s}$, and the scattering angles were given. Similar cuts as used in DATSUM were avallable. Since we knew which of the final events were inelastic and which were elastic we could estimate the background. The histograms showed that in the $(\triangle \mathrm{P} / \mathrm{P})$ distributions the inelastic events peaked under the elastic events.

The third method used was based on hand drawn curves of the background in actual runs. From the summed output of one or more runs at the same enhancement, we selected these histograms: the uncut $(\Delta P / P)_{f}$ and $(\Delta \mathrm{P} / \mathrm{P})_{\mathrm{S}}$ histograms, the $(\Delta \mathrm{P} / \mathrm{P})_{\mathrm{s}}$ and $(\Delta \mathrm{P} / \mathrm{P})_{\mathrm{f}}$ histograms with one cut applied to the other $(\triangle \mathrm{P} / \mathrm{P})$ histogram or to the angle correlation. With each of these histograms we drew a background curve under the elastic peak and so were able to estimate the number of elastics in the peak within the cut range. A typical such histogram is shown in Fig. 18. We demanded that the estimates of the elastics from the various histograms agreed within statistics. Then knowing the amount of events passing all cuts we could determine the background. 


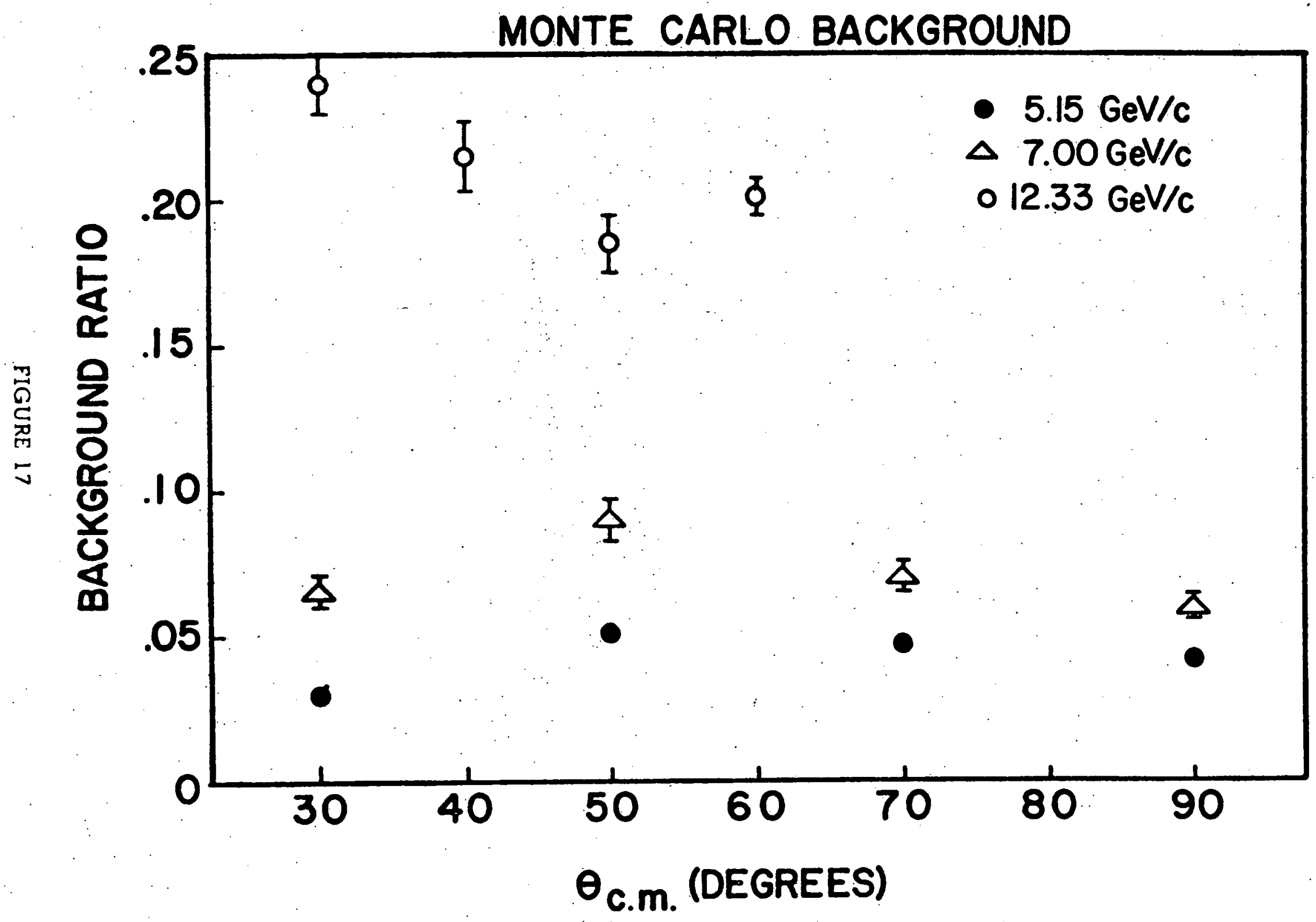




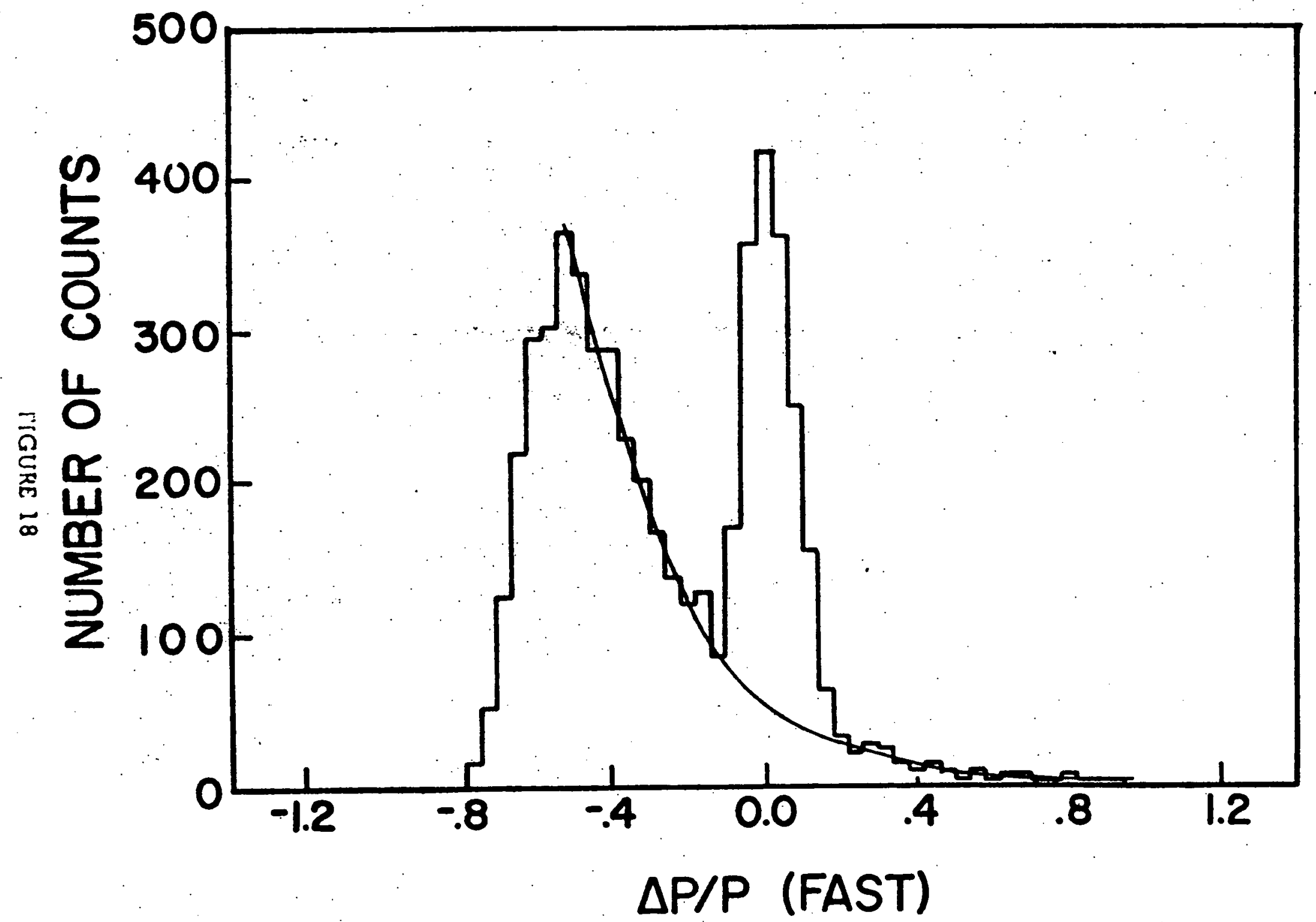


For $5.15 \mathrm{GeV} / \mathrm{c}$ method l's estimate of the background was $13 \%$ (see Fig. 14), method 2, 5\% (see Fig. 17), and method 3, 7\% (see Fig. 19). We had already suspected that during the background running for 5.15 $\mathrm{GeV} / \mathrm{c}$ the cryostat contained water droplets; about $1 \mathrm{gr}$. of water would be necessary to explain the discrepency between method 1 and 3 . We accepted $7 \%$ as the correct maximum and decided to make no correction to the data due to background. For $7 \mathrm{GeV} / \mathrm{c}$ the maximum backgrounds for each method were $13 \%$ method $1,8 \%$ method 2 , and $15 \%$ method 3 . The results of the four angles examined in method 1 for $7 \mathrm{GeV} / \mathrm{c}$ are shown in Fig. 15. For $12.33 \mathrm{GeV} / \mathrm{c}$ the maximum backgrounds for each method were $(46 \pm 21) \%, 23 \%$, and $22 \%$, methods 1,2 , and 3 respectively. The method 1 results for the three angles tested is shown in Fig. 16.

On the basis of these results we decided to make a background subtraction for 7 and $12.33 \mathrm{GeV} / \mathrm{C}$. In the formula for $\mathrm{P}$ we denoted background as $B_{i} \cdot$ In DATSUM we assumed $B_{i}=b(\theta) M_{i}$ where $b(\theta)$ is the background correction factor per monitor count for a given angle as determined by method 3 . Since the background curves drawn in method 3 did not have the peaked shape as shown by the Monte Carlo, we further corrected every $12.33 \mathrm{GeV} / \mathrm{c}$ point by $15 \%$, which was the average difference between the method 3 results and the carbon runs. The reason for doing this was that the method 3 tended to extrapolate a curve mainly produced by particle production under the elastic peak and therefore, could not correct for inelastic scattering off bound protons. The $7.0 \mathrm{GeV} / \mathrm{c}$ results did not need any further correction. 


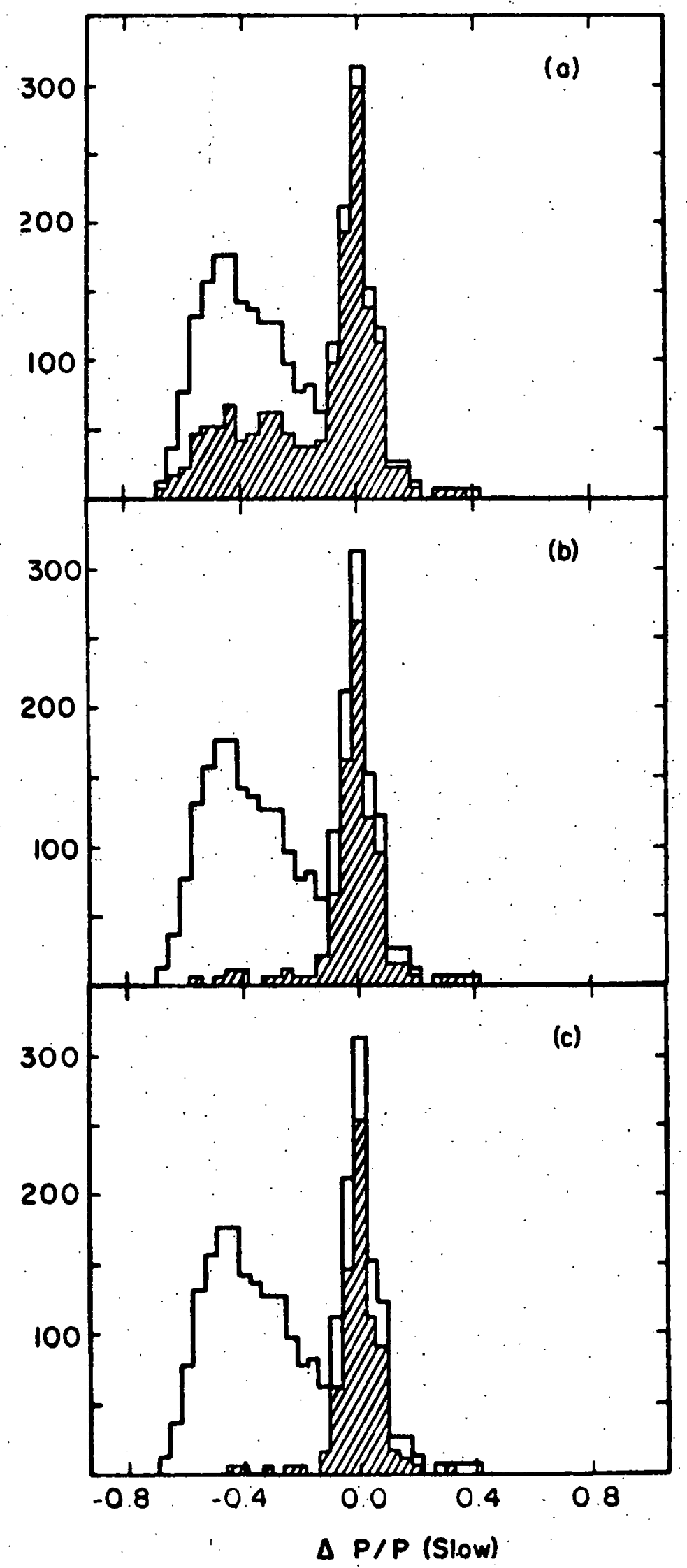

FIGURE 19 


\section{E. Experimental Biases and Errors}

Possible experimental biases could arise from variations in beam position, counter efficiencies, and chamber efficiencies. Fig. 4 shows an event vs. beam position plot for a sweep of the target. From this type of plot and with such plots as in Fig. 5 we could determine the position of the target and the plateau region. The centroid of the beam position was monitored electronically and a warning was given whenever the centroid moved outside of acceptable limits. The limits were determined by the plateau and were typically $1-1.5$ wires from the target center. Frequent changes in target enhancement compensated for slow drifts within the acceptable limits. For every burst HPOS was recorded and during analysis the distribution of HPOS was histogrammed for each run so any run in which HPOS was unusual could be deleted.

The counter and chamber geometry was not changed for the two enhancements of the polarized target. This was because the enhancement change involved pumping in a different microwave frequency, but no change in magnetic field. There was a slight problem in drifts in the field. These drifts were less than .02\% and uncorrelated with the enhancement.

Any slow drifts in counter inefficiencies were compensated for by the frequent enhancement changes; any large drifts were detected by the on-line computer. To correct chamber inefficiencies the number of elastic events reconstructed for each run was corrected by a composite chamber efficiency, represented by $R / A$, calculated for each run. An. 
independent method for calculating this composite efficiency agreed with the number used. In addition, for points with a large number of runs we found that chamber inefficiencies average out, so no correction was necessary.

There are several sources of error that we must consider in our error analysis. These include the error due to statistics, the error in the background subtraction, the error in the polarization of the target due to measurement and to possible localized differences of polarization in the target, the error in any correction factor used, and the experimental asymmetries. The error in the polarization due to the background and the statistics was taken to be $\sqrt{N}$, where $N$ is the number of events or background counts. The statistics for $R$ and $A$ were always much better than for the number of elastics. The error was taken to be insignificant.

The error in measurement of the target polarization was due to two sources. First, the measurement of the average polarization of the target is only good to $5 \%$, primarily because of the TE measurement. Second, the average polarization of the target does not reflect the fact that the target is not uniformly irradiated. Therefore, the more beam put on the target, the larger is the discrepency between the average polarization of the target and the localized polarization.

To get an estimate on the maximum error due to localized differences in polarization, let us assume the beam is one-dimensional and of a Gaussian shape; also assume the beam's center moves uniformly over an interval of .16" (2 wire spacings). Distributions of HPOS for the runs 
show that the beam wandered over 2.5 wire spacings beyond which the distributions drop sharply. This means the beam flux distribution looked like

$$
\begin{aligned}
\operatorname{Flux}(x) & =b \mathrm{e}^{-\mathrm{a}(\mathrm{x}+.08)^{2}} & & x \leq-.08 \\
& =\mathrm{b} & & -.08<x<.08 \\
& =\mathrm{b} \mathrm{e}^{-\mathrm{a}(\mathrm{x}-.08)^{2}} & & x \leq .08
\end{aligned}
$$

where $x=0$ is the center of the target. The full width at half-maximum for the beam was $\geq 9$ wires $=.72 "$. This implies $a=5.36$. If we take $a$ case of target decay from $41 \%$ to $35 \%$, this results in a total flux of 3.51 $\times 10^{13}$ protons assuming the average polarization is given by

$$
P=P_{0} e^{-\varphi / \varphi}
$$

where

$$
P_{0}=.41
$$

and

$$
\varphi_{\mathrm{O}}=2.23 \times 10^{14} \text {, the characteristic flux. }
$$

This allows us to solve for b since $3.51 \times 10^{13}=\int_{-.5}^{.5}$ flux $(x) d x$. The target is 1 " in the $x$-direction; extending the integration over the real line makes the integral trivial to evaluate and can be corrected for by increasing the total flux by $5 \%$. The integral gives

$$
\frac{\mathrm{b}}{2} \sqrt{\frac{\pi}{5.36}}+.16 \mathrm{~b}=3.72 \times 10^{13}
$$

: $11 . \mathrm{b}=6.76 \times 10^{33}$. Now at the center the polarization is then 


$$
\begin{aligned}
P & =.41 \mathrm{e}^{-\left(\frac{.676}{2.23}\right)} \\
& =.31
\end{aligned}
$$

instead of .35 , which is an error of $11 \%$. However this is the error at the end of the life of the target; at the beginning it is $0 \%$; the average is close to $7 \%$. This means that $\mathrm{P}$ is then $\mathrm{P} \pm\left(\sqrt{(.05)^{2}+(.07)^{2}}\right) \mathrm{P}$ giving an error of $\pm .085 \mathrm{P}$.

The results have two errors quoted; one is the statistical error and is plotted; the other is the systematic error. Statistical fluctuations in the number of elastic events and the background, when subtracted, make up the statistical error. The systematic error includes the error due to the polarization of the target, experimental asymmetries, and background when not subtracted, added in quadrature. 


\section{Results and Conclusions}

\section{A. Discussion of Data}

The $5.15 \mathrm{GeV} / \mathrm{c}$ polarization results covered a $|\mathrm{t}|$ range from .6 to $4.0 .(\mathrm{GeV} / \mathrm{c})^{2}$. The polarization vs. $-\mathrm{t}$ is presented in Fig. 20; the polarization vs. $\theta_{\text {c.m. }}$ is plotted in Fig. 21. A tabulation of the polarization along with error is given in Table 2. Fig. 26 has the data plotted along with previous $5.15 \mathrm{GeV} / \mathrm{c}$ data from Ref. 8. In the overlap region. the data from both sources show excellent agreement (data from Ref. 8 were not plotted beyond $\left.t=-.8(\mathrm{GeV} / \mathrm{c})^{2}\right) . \quad$ A dip occurs at $t=-.8(\mathrm{GeV} / \mathrm{c})^{2}$; from there the polarization increases to a maximum of .22 at $t=-1.8$ $(\mathrm{GeV} / \mathrm{c})^{2}$. Then there is a monotonic decline to the $\theta_{\mathrm{c} \cdot \mathrm{m} .}=90^{\circ}$ point at $t=-4.03(\mathrm{GeV} / \mathrm{c})^{2}$ where the polarization is consistent with zero.

$\mathrm{A}|\mathrm{t}|$ range from .6 to $5.3(\mathrm{GeV} / \mathrm{c})^{2}$ was examined at $7.0 \mathrm{GeV} / \mathrm{c}$. From a value of .08 at $\mathrm{t}=-.6(\mathrm{GeV} / \mathrm{c})^{2}$ the polarization rises to a maximum of .20 at $t=-1.7(\mathrm{GeV} / \mathrm{c})^{2}$, then drops to .04 at $t=-3.3(\mathrm{GeV} / \mathrm{c})^{2}$. At larger the polarization remains less than .1 . Fig. 22 shows the polarization as a function of $-t$; Fig. 23 shows the polarization as a function of $\theta$ c.m. All these data are given in Table 3 .

The $12.33 \mathrm{GeV} / \mathrm{c}$ polarization data show more structure than the other two momenta. The measurements start at $t=-1.5(\mathrm{GeV} / \mathrm{c})^{2}$. At $t=-1.9(\mathrm{GeV} / \mathrm{c})^{2}$ the polarization attains a maximum of .20 . Then there is a sudden drop to .02 at $t=-2.3(\mathrm{GeV} / \mathrm{c})^{2}$ followed by a rise to another maximum at $\mathrm{t}=-3.0(\mathrm{GeV} / \mathrm{c})^{2}$. The largest negative polarization occurs 
TABLE 2

POLARIZATION IN P-P ELASTIC SCATTERING AT $5.15 \mathrm{GeV} / \mathrm{C}$

\begin{tabular}{|c|c|c|c|}
\hline${ }^{\theta} \mathrm{c} \cdot \mathrm{m}$. & $-\mathrm{t}\left[(\mathrm{GeV} / \mathrm{c})^{2}\right]$ & Polarization & Error ${ }^{(a)}$ \\
\hline $30^{\circ}$ & .54 & .115 & \pm .018 \\
\hline $35^{\circ}$ & .7 .3 & .076 & \pm .013 \\
\hline $40^{\circ}$ & .94 & .107 & \pm .021 \\
\hline $45^{\circ}$ & 1.18 & .162 & \pm .027 \\
\hline $50^{\circ}$ & 1.44. & .142 & \pm .021 \\
\hline $55^{\circ}$ & 1.72 & .198 & \pm .024 \\
\hline $57^{\circ}$ & 1.84 & .218 & \pm .032 \\
\hline $60^{\circ}$ & 2.02 & .163 & \pm .033 \\
\hline $63^{\circ}$ & 2.20 & .160 & \pm .030 \\
\hline $65^{\circ}$ & 2.33 & .130 & \pm .024 \\
\hline $67^{\circ}$ & 2.46 & .131 & \pm .036 \\
\hline $70^{\circ}$ & 2.65 & .103 & \pm .039 \\
\hline $75^{\circ}$ & 2.99 & .077 & \pm .037 \\
\hline $80^{\circ}$ & 3.33 & .056 & \pm .031 \\
\hline $90^{\circ}$. & 4.03 & .004 & \pm .028 \\
\hline
\end{tabular}

(a) Statistical exror only. Systematic error for every angle is $10 \%$ of the polarization. 


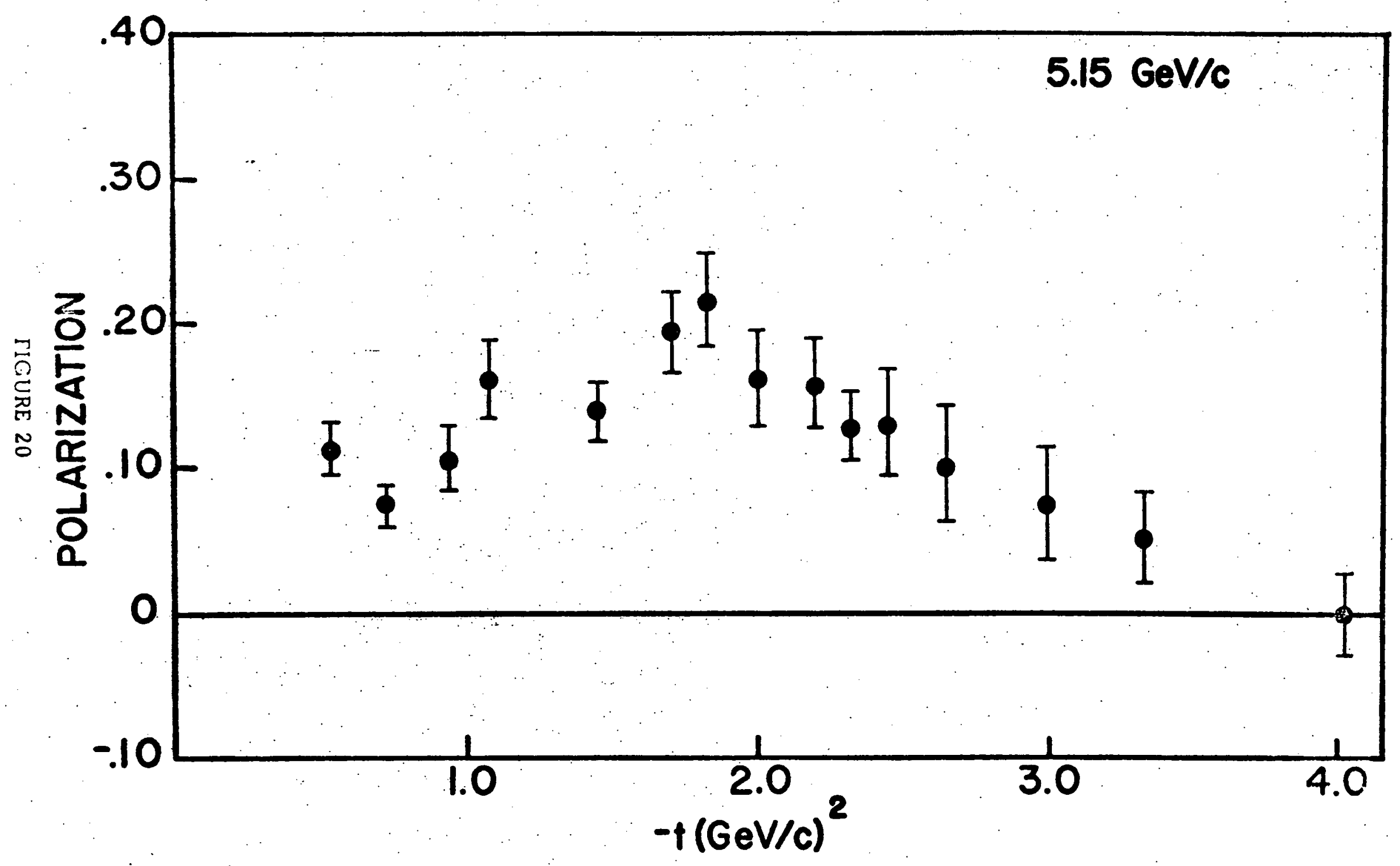




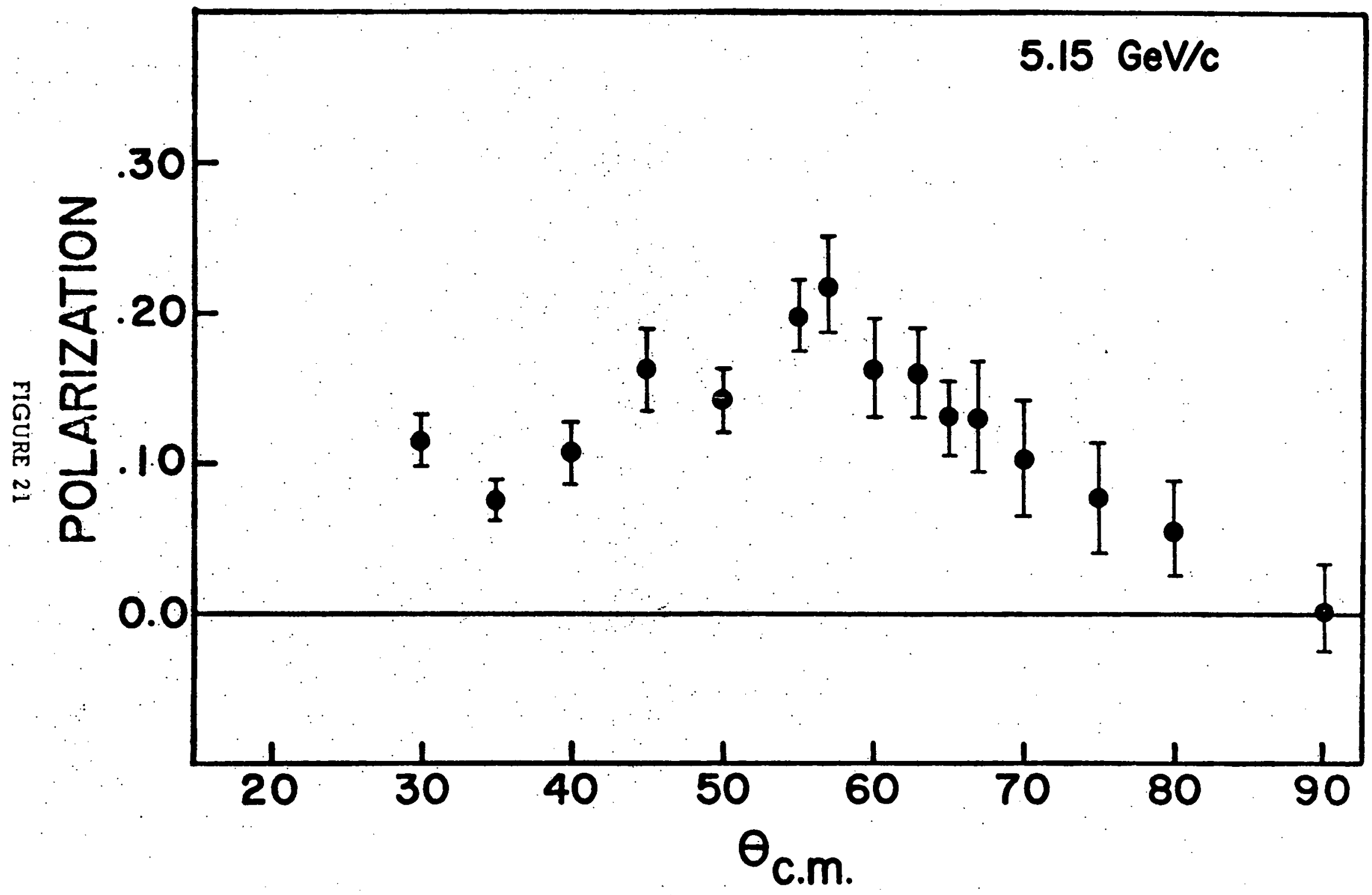


TABLE 3

POLARIZATION IN P-P ELASTIC SCATTERING AT $7.0 \mathrm{GeV} / \mathrm{C}$

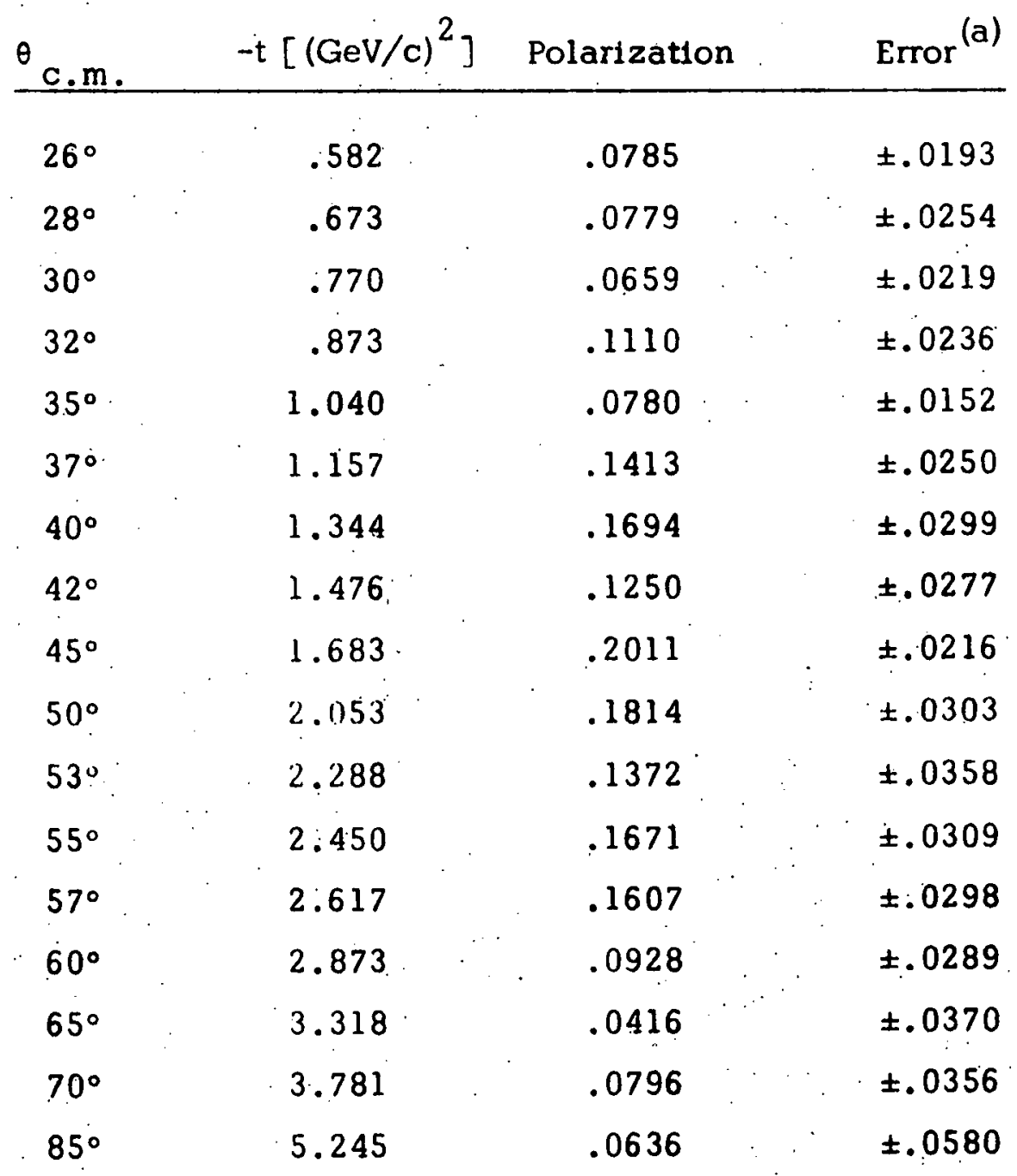

(a) Statistical error only. Systematic error for every angle is $10 \%$ of the polarization. 


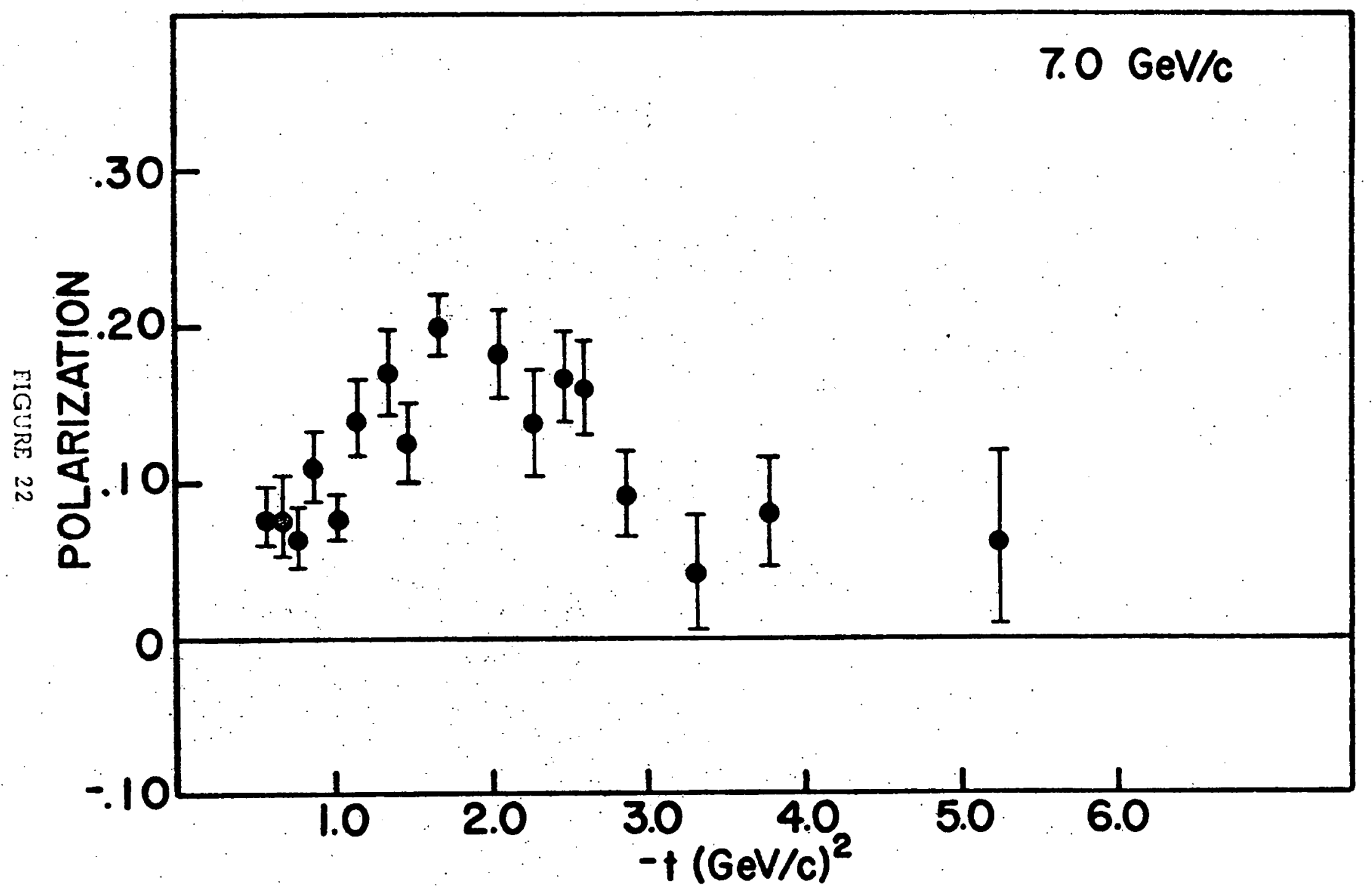




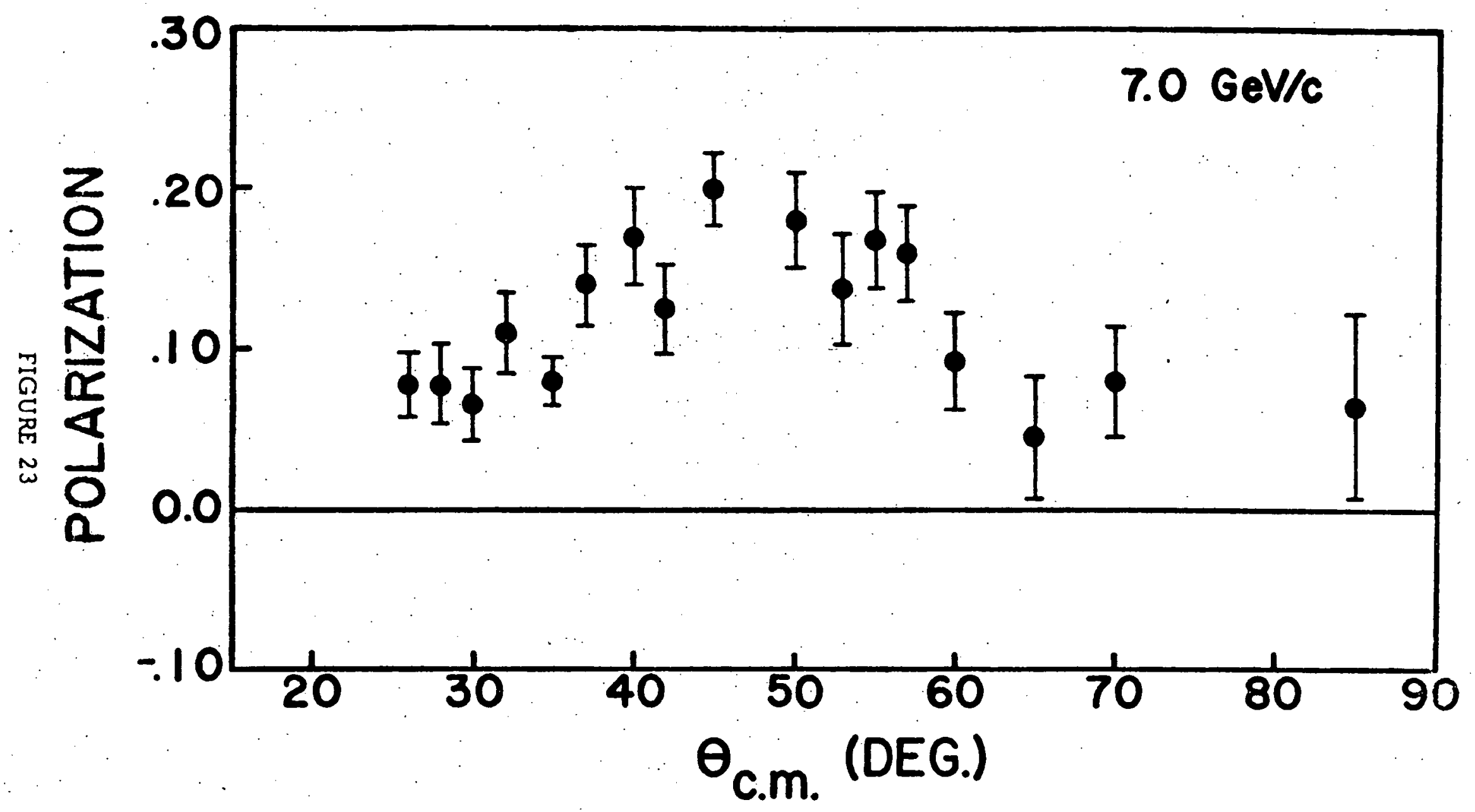

wै 
TABLE 4

POLARIZATION IN P-P ELASTIC SCATTERING AT $12.33 \mathrm{GeV} / \mathrm{C}$

\begin{tabular}{lccc}
$\theta_{\mathrm{c} . \mathrm{m} .}$ & $-\mathrm{t}\left[(\mathrm{GeV} / \mathrm{c})^{2}\right]$ & Polarization & Error \\
\hline $31^{\circ}$ & 1.531 & .1405 & \pm .0434 \\
$33^{\circ}$ & 1.730 & .1995 & \pm .0390 \\
$35.2^{\circ}$ & 1.960 & .1886 & \pm .0385 \\
$36.5^{\circ}$ & 2.103 & .1082 & \pm .0531 \\
$37^{\circ}$ & 2.159 & .0762 & \pm .0556 \\
$38^{\circ} 5^{\circ}$ & 2.331 & .0153 & \pm .0621 \\
$40^{\circ}$ & 2.508 & .0336 & \pm .0532 \\
$41^{\circ}$ & 2.630 & .0476 & \pm .0599 \\
$42^{\circ}$ & 2.754 & .1290 & \pm .0523 \\
$43.5^{\circ}$ & 2.945 & .1641 & \pm .0721 \\
$45^{\circ}$ & 3.140 & -.0082 & \pm .0589 \\
$50^{\circ}$ & 3.830 & -.0069 & \pm .0829 \\
$55^{\circ}$ & 4.572 & -.0919 & \pm .1158 \\
$60^{\circ}$ & 5.361 & .0857 & \pm .1169 \\
$65^{\circ}$ & 6.191 & .2768 & \pm .1391
\end{tabular}

(a) Statistical error only. Systematic error for every angle is $13 \%$ of the polarization. 


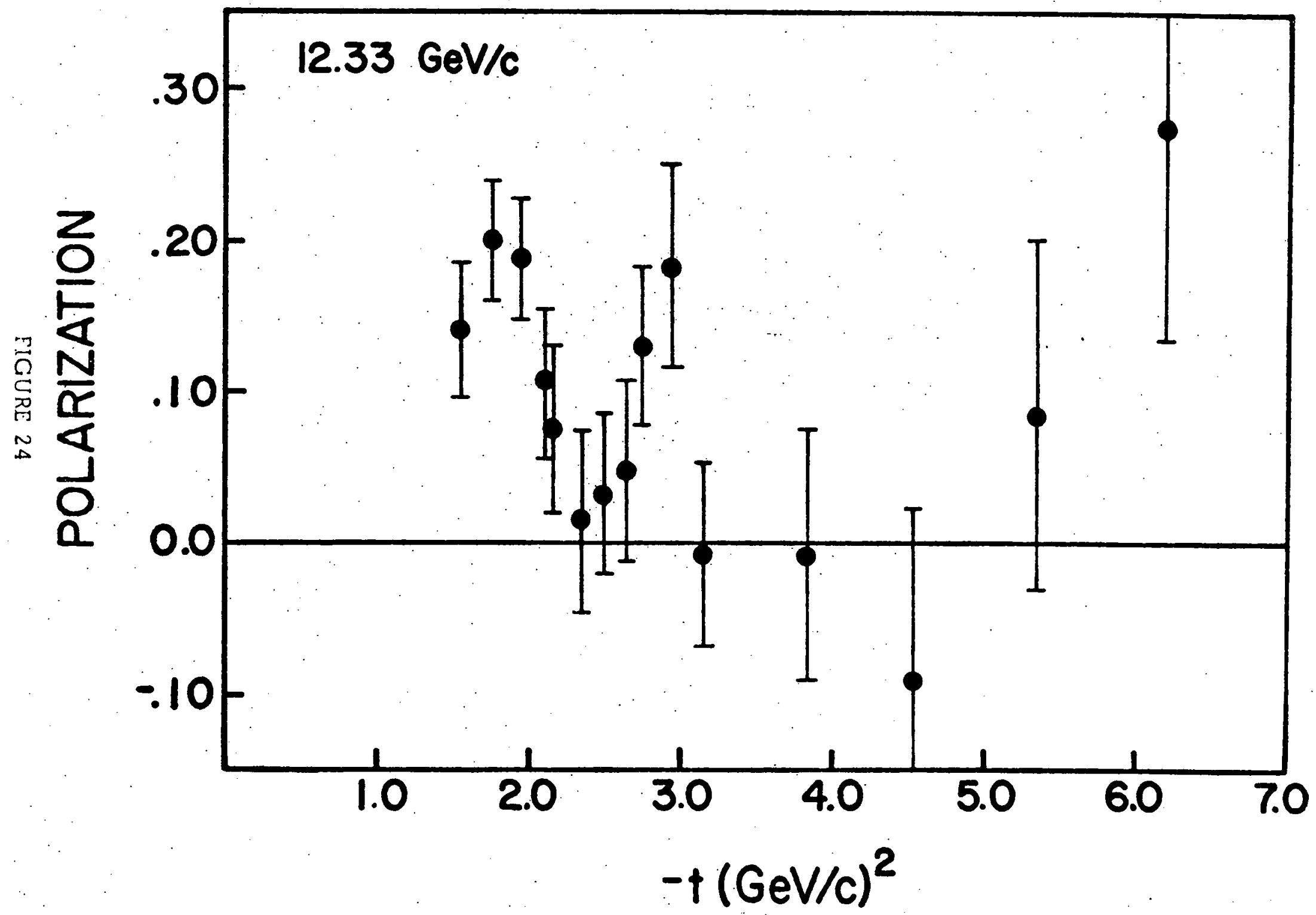




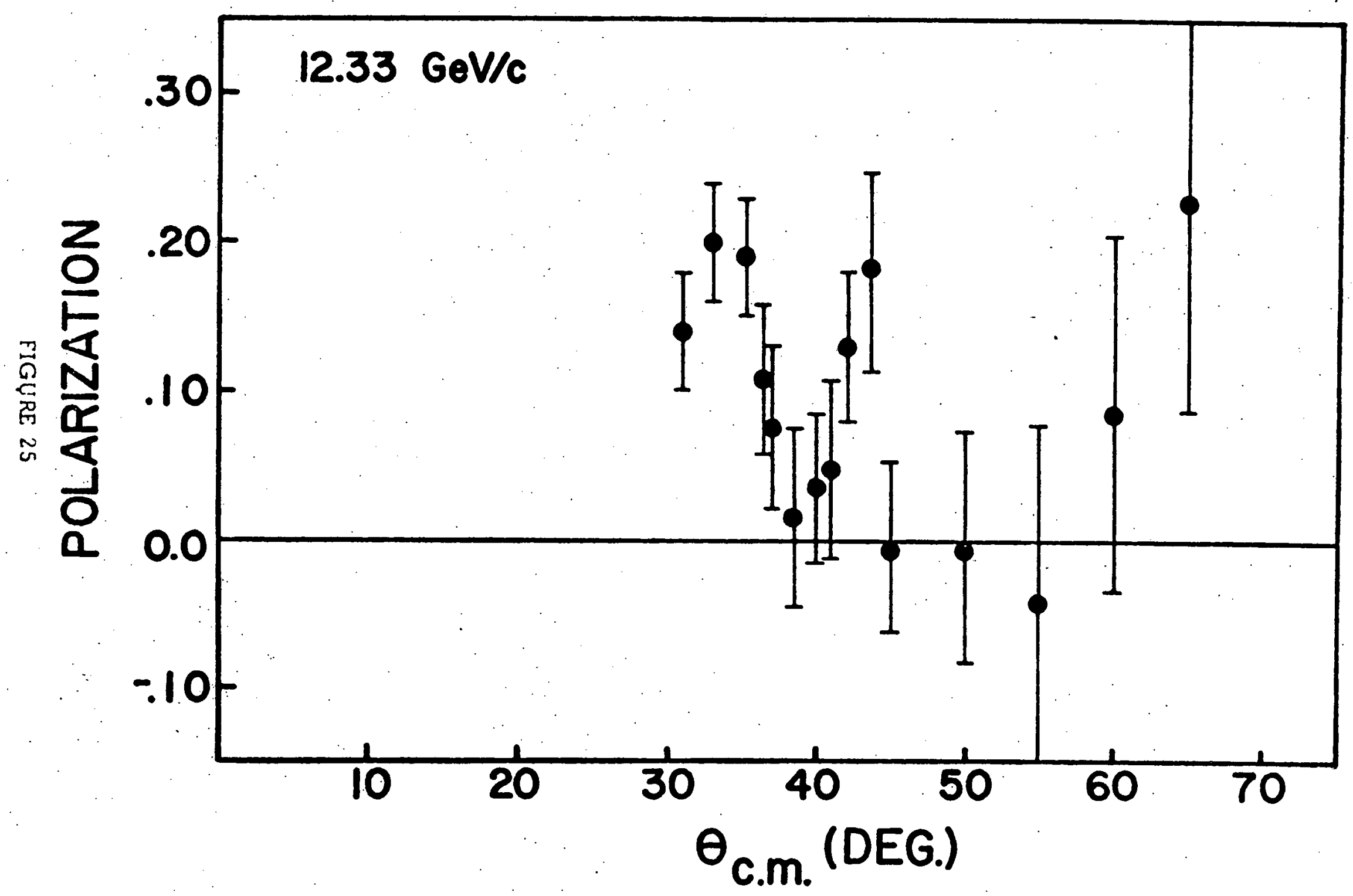


at $t=-4.6(\mathrm{GeV} / \mathrm{c})^{2} ;$ afterwards the polarization rises to a value of .28 at $t=-6.2(\mathrm{GeV} / \mathrm{c})^{2}$ (the last point measured). These polarization

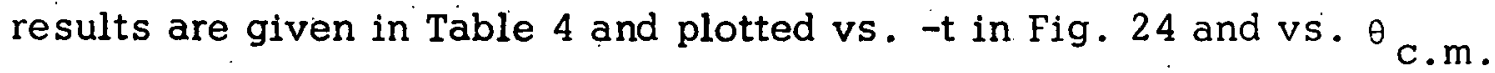
in Fig. 25.

There are some strong correlations in the data. Both the 5.15 and $7.0 \mathrm{GeV} / \mathrm{c}$ results show evidence of a dip at $t=-.8(\mathrm{GeV} / \mathrm{c})^{2}$. All three momenta show a pronounced maximum of about .20 in the vicinity of $t=-1.7(\mathrm{GeV} / \mathrm{c})^{2}$. From $t=-2.0(\mathrm{GeV} / \mathrm{c})^{2}$ to $t=-4.0(\mathrm{GeV} / \mathrm{c})^{2}$, the $5.15 \mathrm{GeV} / \mathrm{c}$ polarization shows a possible change in slope; in the 7.0 $\mathrm{GeV} / \mathrm{c}$ data there is a definite slope change and even the possibility of a dip; this dip definitely appears at $12.33 \mathrm{GeV} / \mathrm{c}$ data at about $\mathrm{t}=-2.4$ $(\mathrm{GeV} / \mathrm{c})^{2}$

B. Discussion of Results in Terms of Models

The results were fitted using three of the four models discussed earlier. In the following we discuss the forms used in the fitting and discuss how well each model fits the data.

The Regge model fits were done using the form of Austin, Greiman; and Rarita. ${ }^{28}$ The $t$-channel helicity amplitudes were parameterized. The forms of the amplitudes are:

$$
\begin{aligned}
& \Phi_{1}=\Phi_{3}=\sum_{1} \xi_{i} f_{s s}^{i}(t) \nu \alpha_{i}(t) \\
& \Phi_{2}=-\Phi_{4}=-\sum_{i} \xi_{1} f_{n n}^{i}(t) v \alpha_{i}(t) \\
& \Phi_{5}=\sum_{i} \xi_{i} f_{n s}^{i}(t) v \alpha_{i}(t)
\end{aligned}
$$


where

$$
\begin{aligned}
& \xi_{i}=\left( \pm 1+\left[\exp -i \pi \alpha_{i}(t)\right]\right) \Gamma\left(1-\alpha_{i}(t)\right) / \pi \\
& =\text { signature factor } \\
& f_{s s}^{i}(t)=\left(1-t / 4 M^{2}\right)\left(b_{s}^{i} \exp \left[c_{s}^{i} t\right]\right)^{2} g_{s s}^{i} \\
& f_{n n}^{i}(t)=\left(-t / 4 M^{2}\right) /\left(1-t / 4 M^{2}\right)\left(b_{n}^{i} \exp \left[c_{n}^{i} t\right]\right)^{2} g_{n n}^{i} \\
& \left.f_{n s}^{i}(t)=\sqrt{-t / 4 M^{2}} b_{s}^{i} b_{n}^{i} \exp L\left(c_{s}^{i}+c_{n}^{i}\right) t\right] g_{s n}^{i} \\
& =\text { residues } \\
& \nu=(s-u) / 4 M^{2} \\
& \alpha_{i}(t)=\alpha_{i}(0)+\alpha_{i}^{i}(0) t \\
& =\text { trajectory }
\end{aligned}
$$

The g's are ghost killing mechanisms which satisfy the condition

$$
g_{s s}^{i} g_{n n}^{i}=\left(g_{s n}^{i}\right)^{2}
$$

The sum is over the poles we used; these were the Pomeranchuk (P), $\mathrm{P}^{\prime}$, $w$, and $w^{\prime}$. $P$ and $\mathrm{P}^{\prime}$ had the signature factor with +1 and the ghost killing mechanism was the no-compensation mechanism with $g_{s s}=g_{n n}=g_{s n}=$ $\alpha(t)$; the $\omega$ and $\omega^{\prime}$ poles had signature factor -1 and the sense-choosing mechanism with $g_{s s}=1 / \alpha(t), g_{n n}=\alpha(t)$, and $g_{n s}=1$. Following the form used by Austin et al, the $\omega^{\prime}$ contribution was subtracted in the sum.

The observables are defined as follows:

$$
\begin{aligned}
& \sigma_{\mathrm{T}}^{\prime}=\operatorname{Im} \Phi_{1}(\mathrm{t}=0) / \mathrm{P}_{\mathrm{lab}} \\
& \mathrm{d} \sigma / \mathrm{dt})_{\text {ela stic }}=\left(.226 / \mathrm{P}_{1 \mathrm{ab}}\right)^{2}\left(\left|\Phi_{1}\right|^{2}+\left|\Phi_{2}\right|^{2}+2\left|\Phi_{5}\right|^{2}\right) \\
& \text { Polarization }=-\frac{2 \operatorname{Im}\left[\Phi_{5} *\left(\Phi_{1}+\Phi_{2}\right)\right]}{\left(\left|\omega_{1}\right|^{2}+\left|\Phi_{2}\right|^{2}+\left|\omega_{5}\right|^{2}\right)}
\end{aligned}
$$




$$
\operatorname{Re} / \operatorname{Im}=\operatorname{Re} \Phi_{1}(t=0) / \operatorname{Im} \Phi_{1}(t=0)
$$

The .226 is a conversion factor from $\mathrm{GeV} / \mathrm{c}$ to $\sqrt{\mathrm{mb}}$ used in the differential cross section.

For each pole there are six unknowns, $\alpha_{i}(0), \alpha_{i}^{\prime}(0), b_{s}^{i}, b_{n^{\prime}}^{i} c_{s}^{i}$, and $c_{n}^{i}$. This makes a total of 24 variables. We have decreased that to 20 by setting $\alpha(0)=1$ for the P pole, $\alpha^{\prime}(0)$ equal for the other three poles, and $\alpha(0)$ equal for the $P^{\prime}$ and $\omega$ poles. The last two were done following a suggestion of Barger and Cline. 29

We made five fits: a separate fit to each momentum, one using 5.15 and $12.33 \mathrm{GeV} / \mathrm{c}$, and one using all three momenta. Table 5 provides all the final values of the parameters. Each fit for this and the other two theories was done to the polarization, differential cross section, total cross section, and real to imaginary ratio of the forward scattering amplitude. The data used is given in captions $26-28$.

The first fit using only the $5.15 \mathrm{GeV} / \mathrm{c}$ data is shown as curve a of Fig. 26. The fit is certainly very adequate. The $P, P^{\prime}$, and $\omega$ poles have approximately equal contributions; however the $\omega$ ' pole's contribution is much smaller due to the large $c_{n}$ value. The second fit was only the 7.0 GeV/c data and is shown in curve a of Fig. 27. Beyond $t=-.6$ $(\mathrm{GeV} / \mathrm{c})^{2}$ the fit to the polarization is reasonable: The larger $x^{2}$ per point is mainly due to a less than adequate fit to the differential cross section. In general, the parameters for this fit.are about the same as for the first fit. The parameters for the third fit using only the $12.33 \mathrm{GeV} / \mathrm{c}$ data are very different. For any pole the fit is only sensitive to the sign of $b_{n} b_{s}$, 


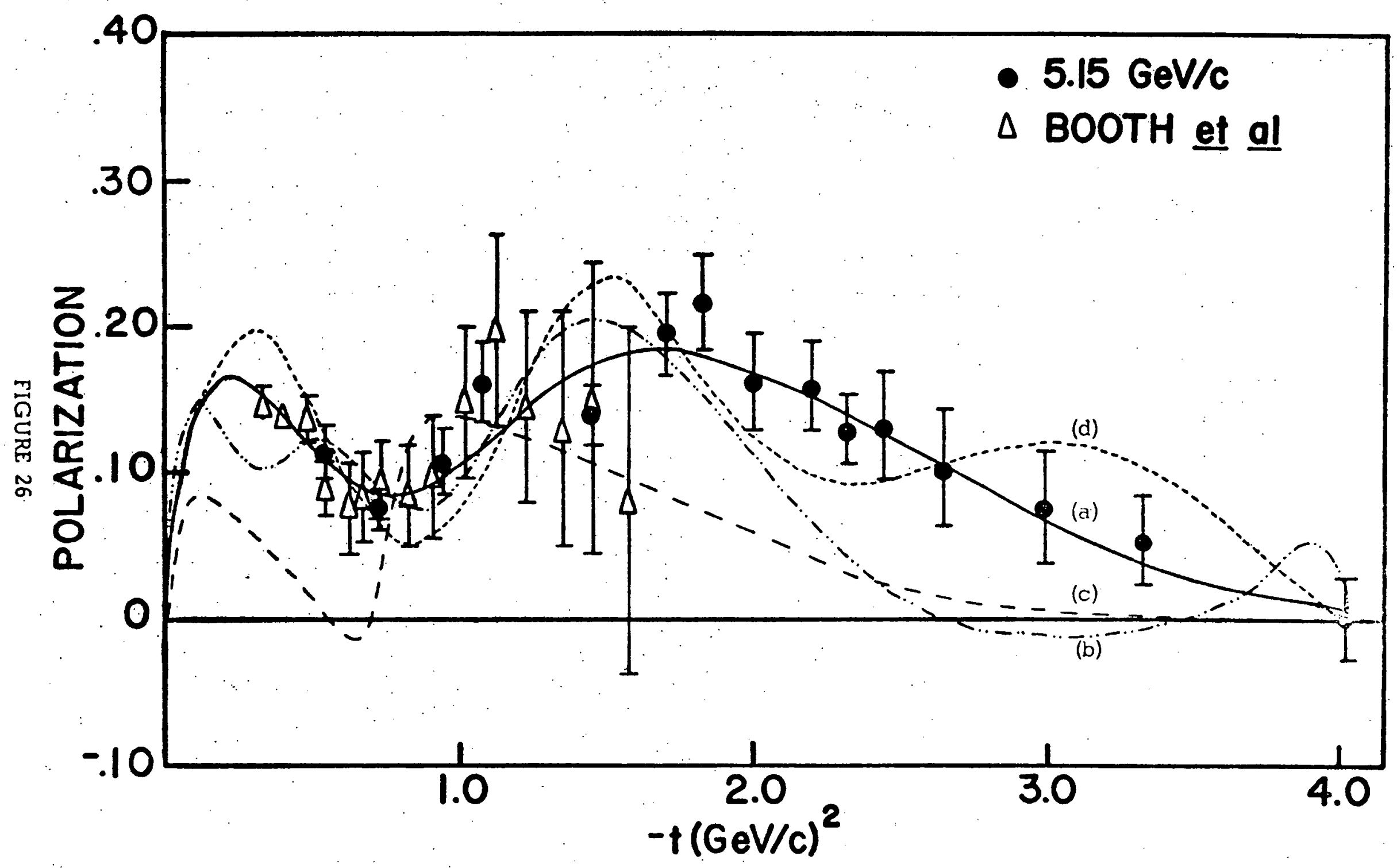




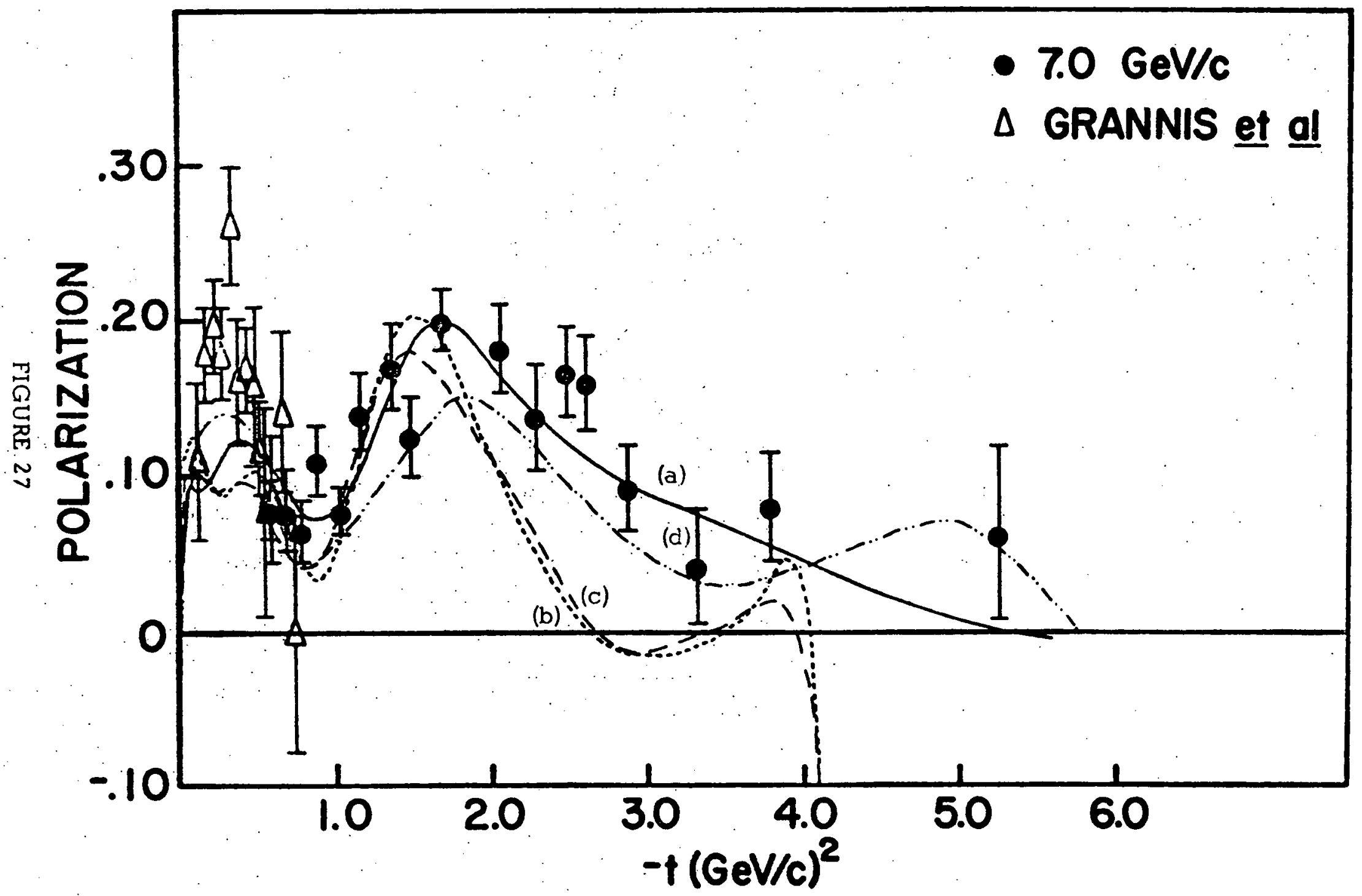




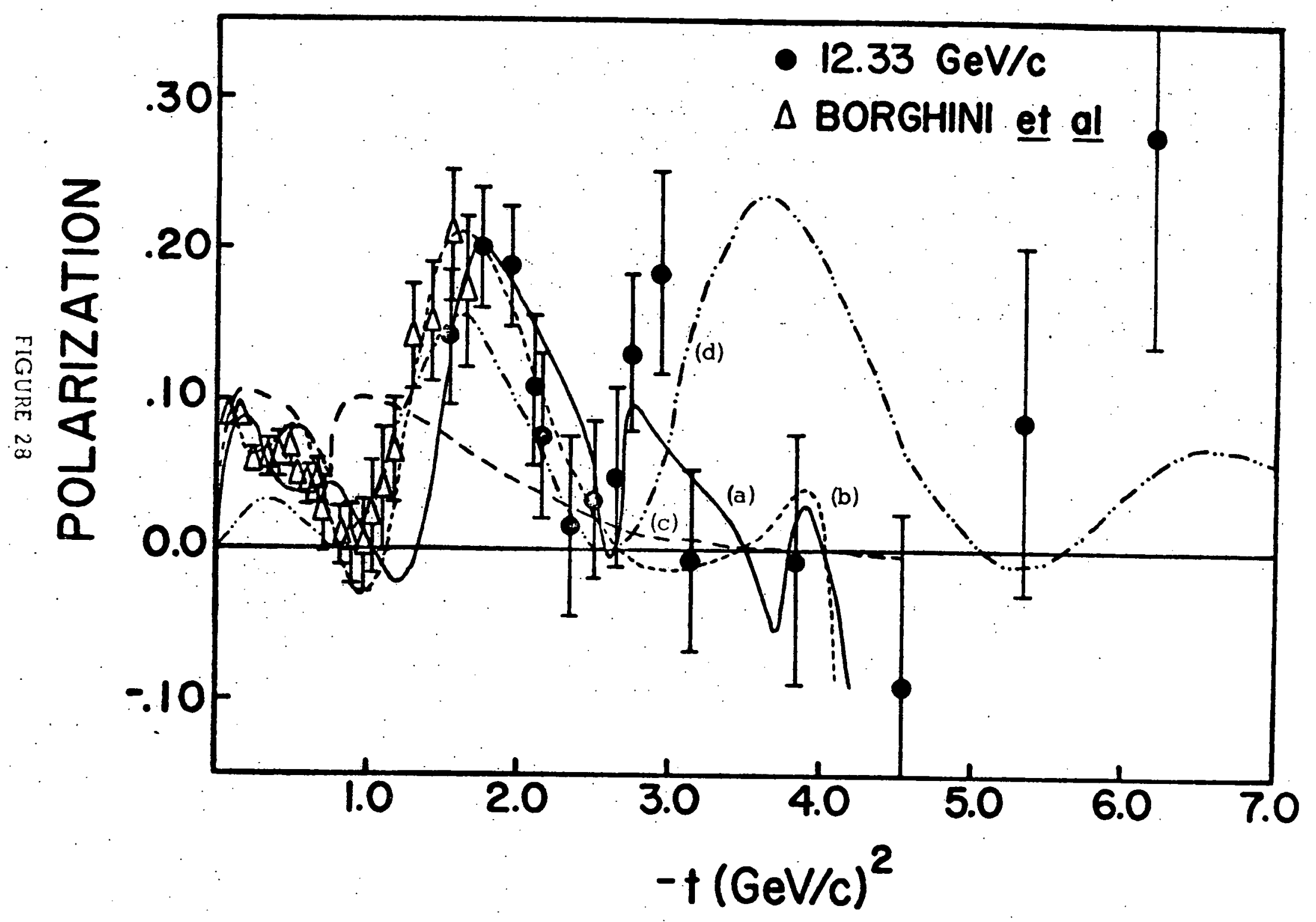


TABLE 5

REGGE POLE PARAMETERS

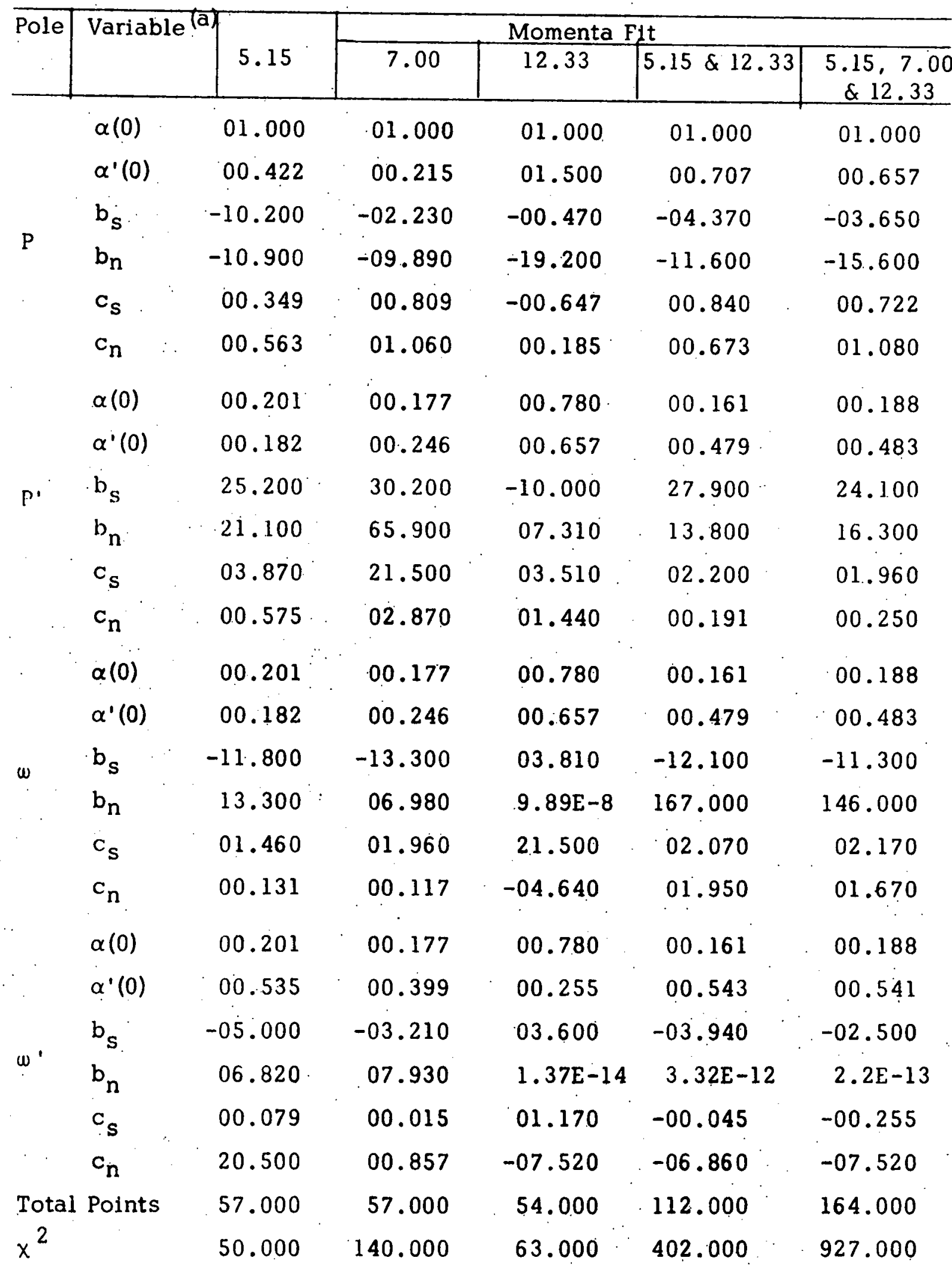

(a) $\alpha(0)$ is unitless; $\alpha^{\prime}(0), c_{s^{\prime}}$ and $c_{n}$ are in $(\mathrm{GeV} / \mathrm{c})^{-2} ; b_{s}$ and $b_{n}$ are in
$(\mathrm{mb} \mathrm{GeV} / \mathrm{c}){ }^{1 / 2}$. 
not to the sign of $b_{s}$ or $b_{n}$ alone. The $P^{\prime}, w$, and $w$ ' poles for the third fit have a different sign for $b_{s} b_{n}$ from the sign for the first two fits. The fit, as shown in curve a of Fig. 28, reproduces the dips at $t=-1.2$ and $-2.6(\mathrm{GeV} / \mathrm{c})^{2}$. However after that, the curve oscillates. There are insufficient constraints in differential cross section or in polarization beyond $t=-4.0$ to prevent these oscillations. The oscillations are a manifestation of this Regge theory parameterization's inability to properly reproduce the structure caused by the first two minima.

The fourth fit to both the 5.15 and $12.33 \mathrm{GeV} / \mathrm{c}$ data was done solely to predict the polarization at $7.0 \mathrm{GeV} / \mathrm{c}$. This fit is shown as curve $c$ in Fig. 27. The fifth fit uses all the data. The fits are shown as curve b in Fig. 26 for $5.15 \mathrm{GeV} / \mathrm{c}$, Fig. 27 for $7.0 \mathrm{GeV} / \mathrm{c}$, and Fig. 28 for 12. $33 \mathrm{GeV} / \mathrm{c}$.. All three curves are of the same general character. They do predict a dip near $t=-.8(\mathrm{GeV} / \mathrm{c})^{2}$; however, the following maximum is not at the correct place. The minimum at $t=-3.0(\mathrm{GeV} / \mathrm{c})^{2}$ cannot be forced to lower $t$. Beyond $t=-4.0(\mathrm{GeV} / \mathrm{c})^{2}$ insufficient constraints make the fit unreliable. $x^{2}$ per do/dt point for each fit $\sim 1$.

The Durand and Lipes model had two amplitudes. The two amplitudes used as inputs the empirical fit to the measured proton form factor,

$$
G_{A}(t)=G_{B}(t)=G_{p}(t) \doteq\left(u^{2} /\left(u^{2}-t\right)\right)^{2}, u^{2}=.71(\mathrm{GeV} / \mathrm{c})^{2}
$$

In terms of the helicity amplitudes, the parameterization is:

$$
\begin{aligned}
\Phi_{1}(s, t) & =\Phi_{3}(s, t)=\frac{1}{k^{2}} \sum_{J=0}^{40} J\lfloor 1-S(J / k)] P_{J}(\cos \theta) \\
& =\text { non-flip term }
\end{aligned}
$$




$$
\begin{aligned}
& \Phi_{2}(s, t)=\Phi_{4}(s, t)=0 \\
& \Phi_{5}(s, t)=\frac{C}{k^{2}} \sum_{J=0}^{40} S(J / k)(u J / k)^{4} K_{3}(u J / k) \sin \theta / 2 \\
& \left.L P_{J}^{\prime}(\cos \theta)+P_{J-1}^{\prime}(\cos \theta)\right] \\
& \quad=\text { flip term }
\end{aligned}
$$

where

$$
\begin{aligned}
& S(x)=\exp \left[-A(x / 2)^{3} K_{3}(x)\right] \\
& A=a_{1}+i a_{2} \\
& C=\left(c_{1}+i c_{2}\right)\left(c_{3}+c_{4} / s\right)
\end{aligned}
$$

This model has six variables.

The observables are

$$
\begin{aligned}
& \mathrm{d} \sigma / \mathrm{dt}=\frac{\pi}{(1.5812)^{2}}\left(\left|\Phi_{1}\right|^{2}+2\left|\Phi_{5}\right|^{2}\right) \\
& \text { Polarization }=-2 \operatorname{Im}\left(\Phi_{5} \Phi_{1}\right) / \mathrm{d} \sigma / \mathrm{dt} \\
& \sigma_{\mathrm{TOT}}=\frac{4 \pi}{(1.5812)^{2}} \operatorname{Im} \Phi_{1}(t=0) \\
& \operatorname{Re} / \operatorname{Im}=\operatorname{Re} \Phi_{1}(t=0) / \operatorname{Im} \Phi_{1}(t=0)
\end{aligned}
$$

The 1.5812 term is the conversion factor such that $\sqrt{\mathrm{mb}} \mathrm{GeV} / \mathrm{c}=1.5812$.

A fit to all the data is shown as curve $\mathrm{c}$ in Fig. 26 for the $5.15 \mathrm{GeV} / \mathrm{c}$ data and as curve $\mathrm{c}$ in Fig. 28 for the $12.33 \mathrm{GeV} / \mathrm{C}$ data. The values of the parameters used are:

$$
\begin{aligned}
& a_{1}=1.2250 \\
& a_{2}=-.0390 \\
& c_{1}=.2078 \\
& c_{2}=-10.7648 \quad(\mathrm{GeV} / \mathrm{c})^{2}
\end{aligned}
$$




$$
\begin{aligned}
& c_{3}=.0098 \\
& c_{4}=.0548
\end{aligned}
$$

The fit produces a dip in the polarization in the region of $t=-.8(\mathrm{GeV} / \mathrm{c})^{2}$. Otherwise the curve does not reproduce the structure seen at either momentum. This fit was done according to the method outlined in Ref. 15. The value of $u^{2}$ was taken to be the experimental value .71. Undoubtedly the fit could be improved by allowing $u^{2}$ to vary in some limited range. Another possible improvement would be to allow $\Phi_{3}$ to have the same form as $\Phi_{1}$ with different parameters. However it is unlikely ${ }^{40}$ that the theory will ever produce the dip at $t=-2.5(\mathrm{GeV} / \mathrm{c})^{2}$.

The fit of the model of Hendry and Chu, the forms of the s-channel helicity amplitudes are:

$$
\begin{aligned}
& \Phi_{1}(s, t)=\frac{1}{k} \sum_{j=0}^{60}(J+1 / 2) \varphi_{1}^{J} d_{00}^{J}(\theta) \\
& \Phi_{2}(s, t)=0 \\
& \Phi_{3}(s, t)=\frac{1}{k} \sum_{J=1}^{60}(J+1 / 2) \varphi_{3}^{J} d_{11}^{J}(\theta) \\
& \Phi_{4}(s, t)=0 \\
& \Phi_{5}(s, t)=\frac{1}{k} \sum_{\sum_{=2} 0}^{60}(J+1 / 2) \varphi_{5}^{J} d_{10}^{J}(\theta) . \\
& d_{\lambda \lambda}^{J},(\theta)=\operatorname{reduced~rotation~matrix~} \\
& \varphi_{1}^{J}=\varphi_{1}(b)=\frac{a_{1}}{l+\exp \left[a_{2}\left(b-a_{3}\right)\right]}+\frac{\text { ia }}{1+\exp \left[a_{5}\left(b-a_{6}\right)\right]}
\end{aligned}
$$




$$
\begin{aligned}
\varphi_{3}^{J} & =\varphi_{3}(b)=\frac{c_{1}}{1+\exp \left[c_{2}\left(b-c_{3}\right)\right]}+\frac{i c_{4}}{1+\exp \left[c_{5}\left(b-c_{6}\right)\right]} \\
\varphi_{5}^{J} & =\varphi_{5}(b)=d_{1} \exp \left[-d_{2}\left(b-d_{3}\right)^{2}\right]+i d_{4} \exp \left[-d_{5}\left(b-d_{6}\right)^{2}\right] \\
& =\text { partial-wave helicity amplitudes } \\
b & =J / k
\end{aligned}
$$

This makes 18 variables. However they impose the tight constraint

$$
\Phi_{1}(s, t=0)=\Phi_{3}(s, t=0)
$$

which effectively reduces the number of variables to 16 . The experimental observables are defined by:

$$
\begin{aligned}
& \frac{\mathrm{d} \sigma}{\mathrm{dt}}=\frac{\pi}{2 \mathrm{k}^{2}}\left(\left|\Phi_{1}\right|^{2}+\left|\Phi_{3}\right|^{2}+4\left|\Phi_{5}\right|^{2}\right) \\
& \mathrm{P}=-\frac{\pi}{\mathrm{k}^{2}} \operatorname{Im}\left[\Phi_{5}{ }^{\star}\left(\Phi_{1}+\Phi_{3}\right)\right] / \mathrm{d \sigma} / \mathrm{dt} \\
& \sigma_{\text {TOT }}=\frac{2 \pi}{\mathrm{k}} \operatorname{Im}\left(\Phi_{1}+\Phi_{2}\right) \mathrm{t}=0 \\
& \operatorname{Re} / \operatorname{Im}=\operatorname{Re} \Phi_{1}(t=0) / \operatorname{Im} \Phi_{1}(t=0)
\end{aligned}
$$

Preliminary fits to the data, provided by Professor A. W. Hendry, using the Chu-Hendry model are shown in Fig. 26 curve $d$ for the 5.15 GeV/c data, in Fig. 27 curve $d$ for the $7.0 \mathrm{GeV} / \mathrm{c}$ data, and in Fig. 28 curve $\mathrm{d}$ for the $12.33 \mathrm{GeV} / \mathrm{c}$ data. The $5.15 \mathrm{GeV} / \mathrm{c}$ curve has the dip at $\mathrm{t}=-.8(\mathrm{GeV} / \mathrm{c})^{2}$. The peak appears at $\mathrm{t}=-1.5(\mathrm{GeV} / \mathrm{c})^{2}$, while the data peaks at a slightly higher $t$. The latter valley and peak are not experimentally observed. However, a possible slope change does occur where the valley occurs. With the exception of being too low, the fit to the 7.0 
TABLE 6

\section{CHUU-HENDRY PARAMETERS}

\begin{tabular}{|c|c|c|c|}
\hline \multirow[t]{2}{*}{ Variable } & \multicolumn{3}{|c|}{ Momentum Fit } \\
\hline & 5.150 & 7.000 & 12.330 \\
\hline$a_{1}$ & -0.400 & -0.330 & -1.640 \\
\hline $\mathrm{a}_{2}(\mathrm{GeV} / \mathrm{c})$ & 4.760 & 1.760 & 1.540 \\
\hline$a_{3}(G e V / c)^{-1}$ & 2.7 .00 & 3.040 & 3.320 \\
\hline$a_{4}$ & 0.534 & 0.496 & 0.473 \\
\hline$a_{5}(\mathrm{GeV} / \mathrm{c})$ & 0.860 & 0.623 & 1.000 \\
\hline$a_{6}(\mathrm{GeV} / \mathrm{c})^{-1}$ & 3.520 & 3.520 & 3.920 \\
\hline $\mathrm{C}_{1}$ & -0.400 & -0.260 & -0.096 \\
\hline $\mathrm{c}_{2}(\mathrm{GeV} / \mathrm{c})$ & 0.910 & 1.500 & 1.400 \\
\hline$c_{3}(\mathrm{GeV} / \mathrm{c})^{-1}$ & 0.910 & 2.250 & 3.000 \\
\hline $\mathrm{c}_{4}$ & 0.230 & 0.238 & 0.249 \\
\hline $\mathrm{c}_{5}(\mathrm{GeV} / \mathrm{c})$ & 0.660 & 0.600 & 1.000 \\
\hline$c_{6} \cdot(\mathrm{GeV} / \mathrm{c})^{-1}$ & 3.600 & 3.600 & 3.820 \\
\hline$d_{1}$ & 0.045 & 0.033 & 0.004 \\
\hline $\mathrm{d}_{2}(\mathrm{GeV} / \mathrm{c})$ & 0.490 & 0.400 & 0.500 \\
\hline$d_{3}(\mathrm{GeV} / \mathrm{c})^{-1}$ & 3.560 & 3.500 & 3.850 \\
\hline $\mathrm{d}_{4}$ & 0.009 & 0.009 & 0.000 \\
\hline $\mathrm{d}_{5}(\mathrm{GeV} / \mathrm{c})$ & 2.900 & 0.390 & $-\because---$ \\
\hline $\mathrm{d}_{6}(\mathrm{GeV} / \mathrm{c})^{-1}$ & 2.460 & 3.100 & --- \\
\hline
\end{tabular}


$\mathrm{GeV} / \mathrm{c}$ data reproduces the data very well. The $12.33 \mathrm{GeV} / \mathrm{c}$ fit also suffers from too low polarization at small $t$. The curve does have a second minimum near $t=-2.5(\mathrm{GeV} / \mathrm{c})^{2}$ followed by a rapid increase, but in this preliminary fit the curve is displaced from the actual data. The fitted parameters to each fit are given in Table 6 . In Table 6 a typical term that is subtracted from $b$ is $3.8(\mathrm{GeV} / \mathrm{c})^{-1}$. In terms of Fermis, 3.8 $(\mathrm{GeV} / \mathrm{c})^{-1}=.8 \mathrm{~F}$. In Chapter 2 , Section $\mathrm{D}$, this term is represented as $\mathrm{f}_{3}$ and $\mathrm{g}_{3}$ and is defined as a radius term. Therefore, the Chu-Hendry model predicts that the radius of the proton in $p-p$ elastic scattering is $.8 \mathrm{~F}$.

\section{Future Expectations}

In addition to providing the first comprehensive data at large $t$ values, this experiment also provides data at smaller $t$ for momenta intermediate between the lower momenta data of Ref. 8 and the higher momenta data of Ref. 31. According to Regge theory ${ }^{41}$ at each value of $t$ the polarization can be expressed as a function of $s$ according to the formula

$$
P=A s^{N}
$$

At various $t$ values ranging from $-.2(\mathrm{GeV} / \mathrm{c})^{2}$ to $-1.8(\mathrm{GeV} / \mathrm{c})^{2}$ we have fitted the available data to this form and calculated the power of $\mathbf{s}$. Sample fitted curves are shown in Fig. 29. The results of these calculations are given in rig. 30. This certainly represents hope for the future of very high energy polarization experiments. The weak dependence on the total c.m. energy in the range of $t \approx-1.5(\mathrm{GeV} / \mathrm{c})^{2}$ will allow nonzero polarization at NAL energies. 


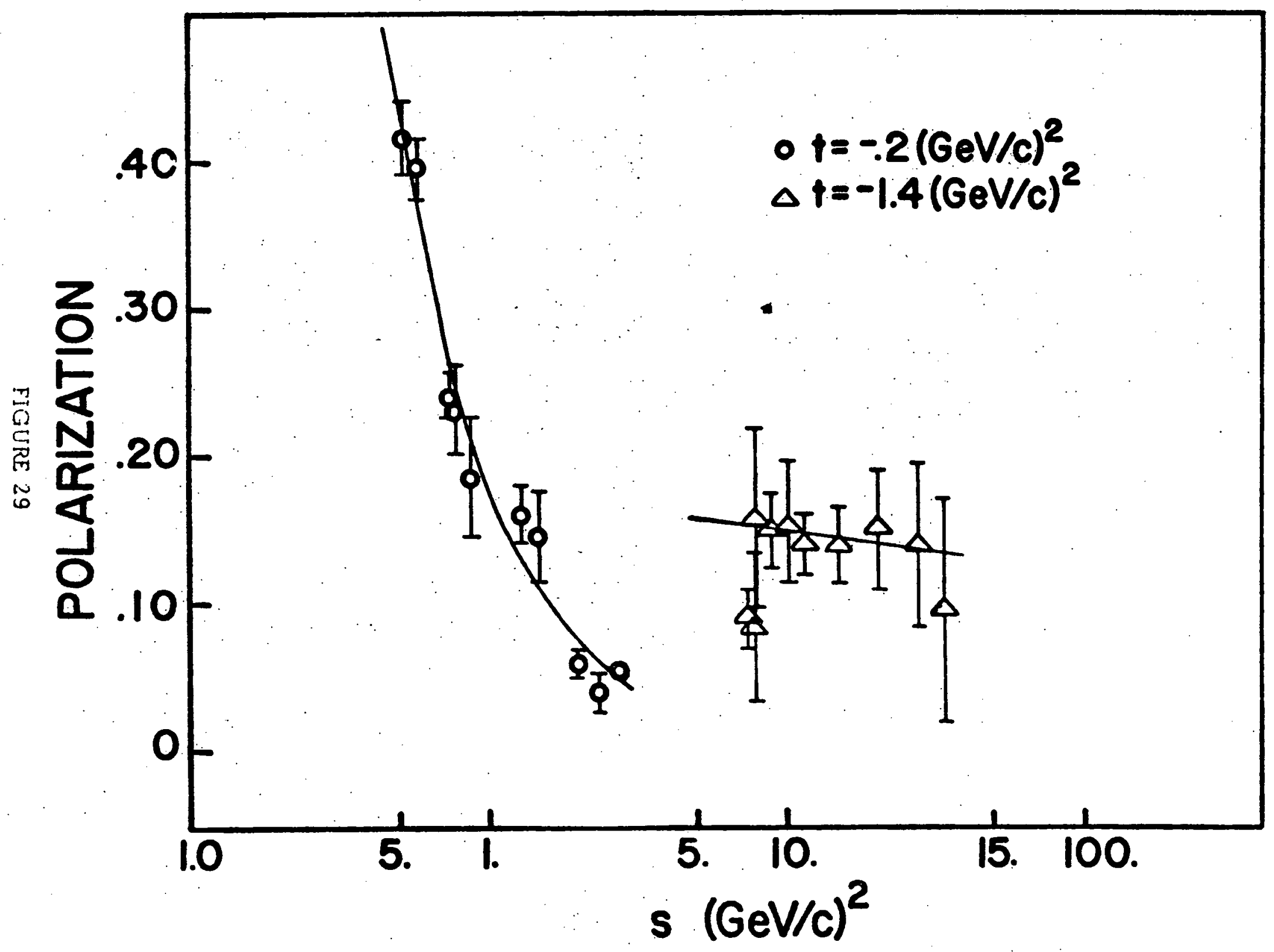

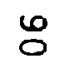




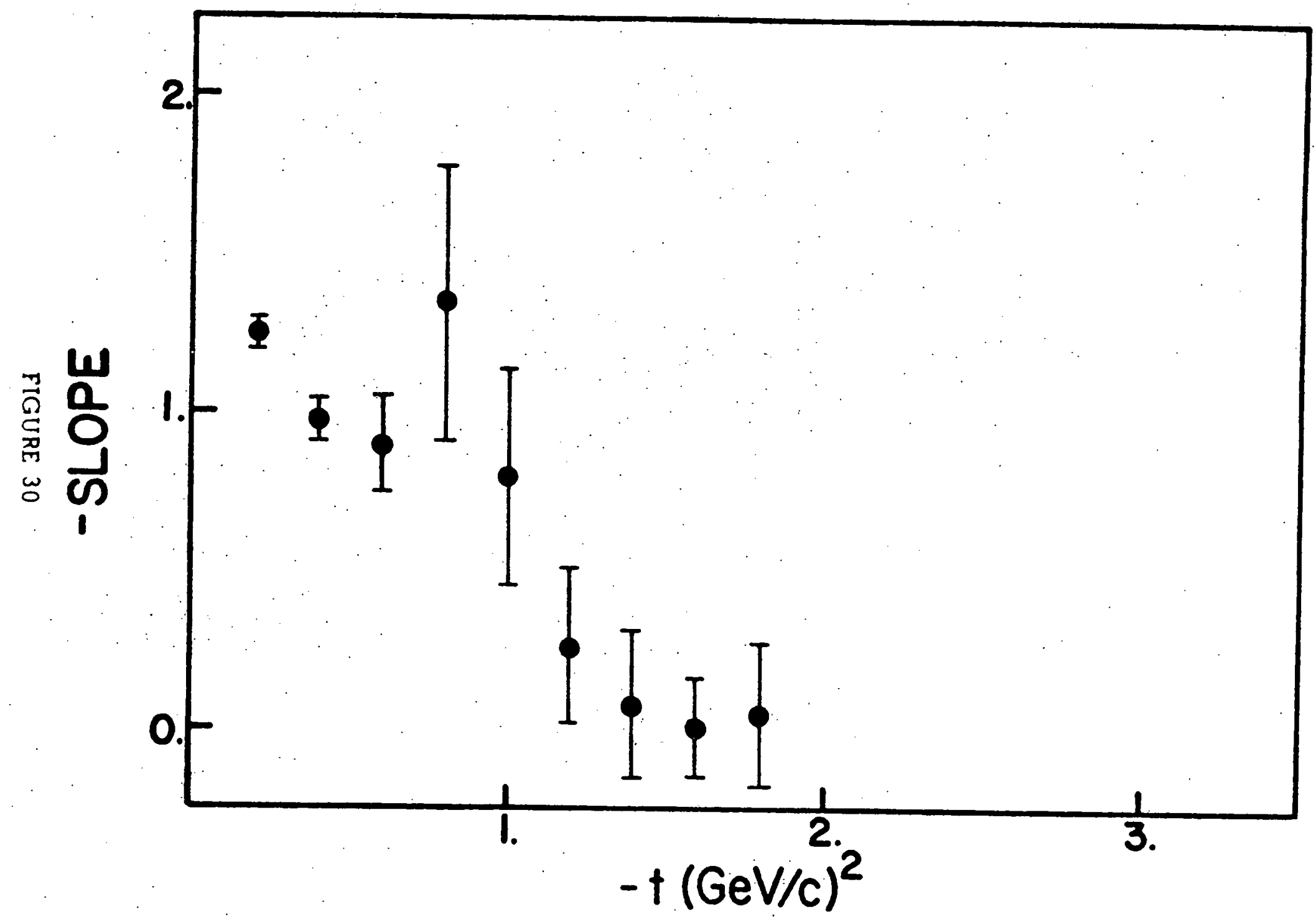


D. Conclusions

This experiment was the first high statistics polarization experiment done at large $t$. The results, especially at $12.33 \mathrm{GeV} / \mathrm{c}$, are not what was expected by those who have predicted little structure in the polarization. It may not be an accident that the slope changes in the $90^{\circ}$ differential cross section at $\mathrm{t}=-.8$ and $-2.5(\mathrm{GeV} / \mathrm{c})^{2}$ occur at the same place as the first two dips in the $12.33 \mathrm{GeV} / \mathrm{c}$ polarization. There is yet no theory that connects them. We believe that the dip in the differential cross section at $t=-1.0(\mathrm{GeV} / \mathrm{c})^{2}$ seen at high energies is definitely correlated with the dip in the polarization seen at $t=-.8$ $(\mathrm{GeV} / \mathrm{C})^{2}$. Both of these dips indicate zeros in the hellcity amplitudes. We expect that at higher energies another dip will be seen near $t=-2.5$ $(\mathrm{GeV} / \mathrm{c})^{2}$ in the differential cross section.

For three theories, fits were done to the polarization, the differential cross section, the total cross section, and the real to imaginary part of the forward amplitude simultaneously. For the $5.15 \mathrm{GeV} / \mathrm{c}$ data the Regge fit to the $5.15 \mathrm{GeV} / \mathrm{c}$ data only and the Chu-Hendry fit are both adequate. Likewise the Regge fit to the $7.0 \mathrm{GeV} / \mathrm{c}$ data only and ChuHendry fit describe the polarization well. Only the Chu-Hendry model can describe the general shape of the data at $12.33 \mathrm{GeV} / \mathrm{c}$. The fourth model, the parton model, predicts no polarization at high energies and large t. Our $12.33 \mathrm{GeV} / \mathrm{c}$ data does not tend to support the prediction. Just a few years ago this experiment would have been considered to be technically unfeasible and physically uninteresting. With the 
advent of higher rate counters and chambers and the development of polarized targets with higher target polarizations, even larger $t$ experiments are now feasible. The new structure seen in this experiment should revive interest in one of nature's most basic systems. 
References

28. D. M. Austin, W. H. Greiman, and W. Rarita, Phys. Rev. D2, 2613 (1970).

29. V. D. Barger and D. B. Cline, Phenomenological Theories of High Energy Scattering (W. A. Benjamin, Inc., New York, 1969), Chapter 5.

30. P. Grannis, J. Arens, F. Betz, O. Chamberlain, B. Dieterle, C. Schultz, G. Shapiro, H. Steiner, L. van Russum, and D. Weldon, Phys. Rev. 148, 1297 (1966).

31. M. Borghini, L. Dick, J. C. Olivier, H. Aoi, D. Cronenberger, G. Grégoire, Z. Janout, K. Kuroda, A. Michalowicz, M. Poulet, D. Sillou, G. Bellettini, P. L. Braccint, T. Del Prete, L. Foá, P. Laurelli, G. Sanguinetti, and M. Valdata, Phys. Letters 36B, 493 (1971).

32. G. C. Fox and C. Quigg, Compilation of Elastic Scattering Data, UCRI-20001:

33. G. Cozzika, Y. Ducros, A. de Lesquen, J. Movchet, J. C. Raoul, L. van Rossum, J. Deregel, and J. M. Fontaine; Phys. Rev. 164, 1672.(1967).

34. M. Borghini, G. Coignet, L. Dick, K. Kuroda, L. Di Lella, P. C. Macq, A. Michalowicz, and J. C. Olivier, Phys. Letters 24B, 77 (1967).

35. D. V. Bugg, D. C. Salter, G. H. Stafford, R. F. George, K. F. Riley, and R. J. Tapper, Phys. Rev. 146, 980 (1966).

36. G. von Dardel, D. H. Frisch, R. Mermod, R. H. Milburn, P. A. Piroué, M. Vivargent; G. Weber, and K. Wenter, Phys. Rev. Letters $\underline{5}, 333$ (1960).

37. A. Ashmore, G. Cocconi, A. N. Diddens, and A. M. Wetherell, Phys. Rev. Letters $\underline{5}, 576$ (1960).

38. A. E. Taylor, A. Ashmore, W. S. Chapman, D. F. Falla, W. H. Range, D. B. Scott, A. Astbury, F. Capocci, and T. G. Walker, Phys. Letters 14, 54 (1965).

39. L. Kirlllova, L. Khristov, V. Nikitin, M. Shafranova, L. Strunov, V. Sviridov, Z. Korbel, L. Rob, P. Markov, Kh. Tchernev, T. Todorov, and A. Zlateva, Phys. Letters 13, 93 (1964): 
40. A. W. Hendry, private communication.

41. I. J. Muzinich, Phys. Rev. Letters 9, 475 (1962). 


\section{List of Figure Captions}

1. Layout at the end of the first $5.15 \mathrm{GeV} / \mathrm{c}$ running. . . . . . 19

2. Pin configuration used for the MC1710G and MC4012P integrated circuits. $D_{P_{N}}$ represents the shift register inputs from the chambers and $\mathrm{Q}_{\mathrm{N}}^{\mathrm{N}}$ the outputs. ............. 24

3. Schematic of the beam line used, not to scale. . . . . . 25

4. Event-event accidental, normalized to the amount of beam septumed from EPBI as given by $\mathrm{ICl} 02$, is plotted against the average horizontal position of the beam. Fig. 4-7 are from a target sweep made during the startup of one of the 12.33 $\mathrm{GeV} / \mathrm{c}$ runs. Shown are typical error bars on HPOS as determined by the full width at half-maximum (FWHM) for a typical run and not for the sweep. Typical statistical errors for the event-event accidental are given. Note that the plateau is from wire 12 to $16(1$ wire $=2 \mathrm{~mm})$. During the subsequent runs with this target the beam was kept between wires 13 and $15 . .28$

5. FA and SA, normalized to IC102, are plotted against HPOS. FA and SA have errors smaller than the size of the symbols. SA peaks at HPOS $=14.5$ in agreement with Fig. 4. FA

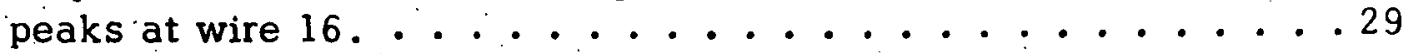

6. BM, normalized to IC 102 , is plotted against HPOS: The plateau is from wire 15 to wire 18; this was before BM was correctly positioned. Since 1 wire $=2 \mathrm{~mm}$, this means we had to move BM $21 / 2$ wire spacings or $5 \mathrm{~mm}$ to center the plateau on wire 14. The error in BM is a round off error in

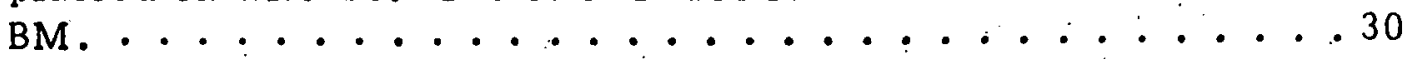

7. $\mathrm{LM}$ and RM, normalized to $\mathrm{ICl} 02$, plotted against HPOS. RM peaks at wire $14 ;$ LM peaks at wire 1.7. Since this was before LM was positioned, we moved it $6 \mathrm{~mm} \ldots \ldots . . . .31$

8. Energy levels of the four possible spin combinations for the proton-radical combination. Transitions (1) and (2) are allowed, (3) and (4) are forbidden. $s_{r}$ is the spin of the

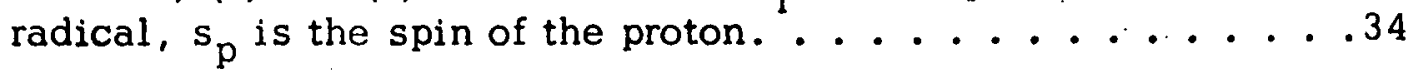

9. Fast logic circuits of the most important components. . . . 36

10. A typical distribution of $(\Delta \mathrm{P} / \mathrm{P})$. This and the next three figures are from a run at $50^{\circ}, 7 \mathrm{GeV} / \mathrm{c}$. The cuts used for 
this run were \pm .18 on the $(\Delta P / P)_{f}, \pm .20$ on $(\triangle P / P)_{S}$ and \pm 1.6 on the angle correlation. The results after cuts are

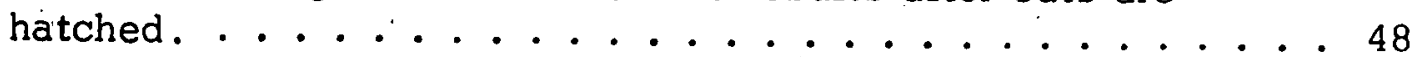

11. A typical distribution of $(\Delta \mathrm{P} / \mathrm{P})_{\mathrm{s}} \ldots \ldots . . . . . . . . . . . . .49$

12. A typical relative TOF distribution. ............. 50

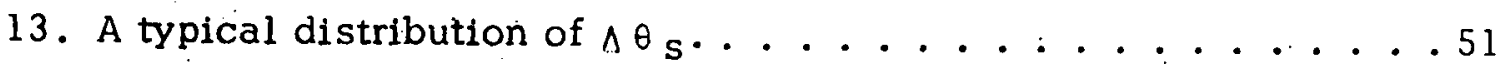

14. Background studies made at $5.15 \mathrm{GeV} / \mathrm{c} ; 14 \mathrm{a}$ is the background in an empty target run; $14 \mathrm{~b}$ is the background due to carbon; $14 \mathrm{c}$. shows a typical run with $14 \mathrm{a}$ and $14 \mathrm{~b}$ summed and displayed as a hatched area. The total background is $13 \%$ of the total. All three runs were made at $55^{\circ}$. . . . . . 55

15. Background studies made at $7.0 \mathrm{GeV} / \mathrm{C}$. The hatched areas are the background due to carbon and the cryostat shown under typical runs. $17 \mathrm{a}$ shows $7.5 \%$ at $32^{\circ} ; 17 \mathrm{~b}$ has $13.3 \%$ at $40^{\circ} ; 17 \mathrm{c}$ has $9.8 \%$ at $50^{\circ} ; 17 \mathrm{~d}$ has $11 \%$ at $60^{\circ} \ldots . . . . .56$

16. Background studies made at $12.33 \mathrm{GeV} / \mathrm{c}$ with carbon. 17a has $(24.1 \pm 6) \%$ background at $40^{\circ} ; 17 \mathrm{~b}$ has $(47 \pm 21) \%$ at $50^{\circ}$; $17 \mathrm{c}$ has $(27.8 \pm 18.5) \%$ at $60^{\circ}$ : Compared to the errors in the background at $12.33 \mathrm{GeV} / \mathrm{c}$, the errors at 5.15 and $7.0 \mathrm{GeV} / \mathrm{c}$ in the background were insignificant. The hatched areas are the background under typical runs. . . . . . . . . 57

17. Monte Carlo results for $p p \rightarrow p p$ scattering from bound protons . The results are shown for $5.15,7.0$ and $12.33 \mathrm{GeV} / \mathrm{c}$ over the range of angles measured in this experiment. . . . . . . 59

18. A typical example of a hand drawn background curve. Note that the curve is extrapolated through the elastic peak using the tails of the distribution. Also note that the curve is not peaked under the elastic peak as the Monte Carlo predicts it should. This curve was from the positive enhancements runs made at $37^{\circ}, 12.33 \mathrm{GeV} / \mathrm{c}$...............60

19. $\Delta \mathrm{P} / \mathrm{P}$ distribution for particles in the slow arm for a typical run at ${ }^{\circ} \mathrm{C} . \mathrm{ml} .=65^{\circ}$ at $5.15 \mathrm{GeV} / \mathrm{c}$. The unshaded curves corresipond to the uncut $\Delta P / P$. The shaded curve in a) 1llus lidlo:; the oflect of the rolative TOF cut. The shaded curves in b) illustrates the combined effect of the TOF cut and a $\Delta \mathrm{P} / \mathrm{P}$ (fast) cut. The shaded curve in c) illustrates the combined effects of the cuts in b) and a $\Delta \theta_{s}$ cut. ........ 62 
20. Polarization data at $5.15 \mathrm{GeV} / \mathrm{c}$ plotted against $-\mathrm{t}$. The errors shown are statistical errors only. . . . . . . . 69

21. Polarization data at $5.15 \mathrm{GeV} / \mathrm{c}$ plotted against $\theta_{\mathrm{c} . \mathrm{m} \text {. The }}$ plotted errors are statistical. ............. 70

22. Polarization data at $7.0 \mathrm{GeV} / \mathrm{c}$ plotted against $-t$. The errors shown are statistical only. ............. . 72

23. Polarization data at $7.0 \mathrm{GeV} / \mathrm{c}$ plotted against $\theta_{\mathrm{c} . \mathrm{m} \text {. The }}$ errors plotted are statistical. ...............73

24. Polarization data at $12.33 \mathrm{GeV} / \mathrm{c}$ plotted against $-\mathrm{t}$. The errors shown are statistical. ........... 75

25. Polarization data at $12.33 \mathrm{GeV} / \mathrm{c}$ plotted against $\theta$. The errors plotted are statistical. . . . . . . . . . . 76

26. Theoretical fits to the $5.15 \mathrm{GeV} / \mathrm{c}$ polarization. The other $5.15 \mathrm{GeV} / \mathrm{c}$ data is from Ref. 8. Curve a is a Regge fit using only the $5.15 \mathrm{GeV} / \mathrm{c}$ data; curve $\mathrm{b}$ is a Regge fit using all three inumonta; curve $c$ is a ftt to the Durand-tipes model using all theo momenta; curve d is a fit to the Chu-Hendry model using the $5.15 \mathrm{GeV} / \mathrm{c}$ data. The differential cross section measurements used were those at $5.0 \mathrm{GeV} / \mathrm{c}$ from Ref. 5 and 32. The total cross section at $5.22 \mathrm{GeV} / \mathrm{c}$ came from Ref. 35. Data at $7.85 \mathrm{GeV} / \mathrm{c}$ from Ref. 38 was used for the $\operatorname{Re} / \mathrm{Im}$ value. ............. 80

27. Theoretical fit to the $7.0 \mathrm{GeV} / \mathrm{c}$ polarization. The other 7.0 $\mathrm{GeV} / \mathrm{c}$ data plotted is from Ref. 30. Curves $\mathrm{a}, \mathrm{b}$, and $\mathrm{c}$ are Regge curves produced by using the $7.0 \mathrm{GeV} / \mathrm{c}$ data only, all three momenta, and the 5.15 and $12.33 \mathrm{GeV} / \mathrm{c}$ data. Curve $\mathrm{d}$ is the Chu-Hendry fit to the $7.0 \mathrm{GeV} / \mathrm{c}$ data. The differential cross section at 7.0 and $7.06 \mathrm{GeV} / \mathrm{c}$ was from Ref. 32. The total cross section at $7.0 \mathrm{GeV} / \mathrm{c}$ was from Ref. 36. The $\mathrm{Re} / \mathrm{Im}$ measurement at $7.85 \mathrm{GeV} / \mathrm{c}$ came from Ref. 38. . . . . . 81

28. Theoretical fit to the $12.33 \mathrm{GeV} / \mathrm{c}$ polarization data. In addition the $10 \mathrm{GeV} / \mathrm{c}$ data from Ref. 31 is plotted. Curve a is a Regge fit to the $12.33 \mathrm{GeV} / \mathrm{c}$ data only; curve $\mathrm{b}$ is a Regge fit to all three momenta; curve $c$ is a fit to the Durand-Lipes model using all three momenta; curve $d$ is a fit to the ChuHendry model using the $12.33 \mathrm{GeV} / \mathrm{c}$ data. Differential cross section data at 12.1 and $12.4 \mathrm{GeV} / \mathrm{c}$ were taken from Ref. 32. Total cross section results at $12.4 \mathrm{GeV} / \mathrm{c}$ came from Ref. 37. The Re/Im measurement at $10.9 \mathrm{GeV} / \mathrm{c}$ was from Ref. 39................. 82 
29. Two sample polarization vs. s (square of the total c.m. energy) curves at $t=-.2$ and $-1.4 \mathrm{GeV} / \mathrm{c}$. The power of $\mathrm{s}$ fitted for the first plot is $-1.238 \pm .045$; for the second plot it is $-.080 \pm .237$. Data in the range $1.23-1.915$ GeV/c came from Ref. 33; the range $2.5-5.15 \mathrm{GeV} / \mathrm{c}$ came from Ref. 8; data at 3.037 and 3.660 was from Ref. 7 ; data from $10.0-17.5 \mathrm{GeV} / \mathrm{c}$ came from Ref. 31 ; and data at 6.0 and $12.0 \mathrm{GeV} / \mathrm{c}$ came from Ref. $34 . . . . . . . . .90$

30. The slopes of the polarization vs. s curves are plotted at each value of $t$. The slope is the exponent in the formula $P=A s^{N}$ 


\section{List of Tables}

Table

Page

l Chamber and Counter Positions........... 20

2 Polarization in P-P Elastic Scattering at $5.15 \mathrm{GeV} / \mathrm{C}$. . . 68

3 Polarization in P-P Elastic Scattering at $7.0 \mathrm{GeV} / \mathrm{c}$. . . . 71

4 Polarization in P-P Elastic Scattering at $12.33 \mathrm{GeV} / \mathrm{c}$. . . . 74

5 Regge Pole Parameters ...............83

6 Chu-Hendry Parameters ............... 88 